TRANSACTIONS OF THE

AMERICAN MATHEMATICAL SOCIETY

Volume 364, Number 8, August 2012, Pages 3915-3965

S 0002-9947(2012)05398-0

Article electronically published on March 21, 2012

\title{
LAPLACIANS ON A FAMILY OF QUADRATIC JULIA SETS I
}

\author{
TARYN C. FLOCK AND ROBERT S. STRICHARTZ
}

\begin{abstract}
We describe families of Laplacians on Julia Sets $\mathcal{J}_{c}$ for quadratic polynomials $P(z)=z^{2}+c$ in the spirit of Kigami's construction of Laplacians on p.c.f. self-similar fractals. We consider an infinite family of Julia sets for $c$ in the interior of a bulb in the Mandelbrot set that includes the basilica and the Douady rabbit. We use the external ray parametrization of the Julia set which represents the Julia set as a circle with some points identified. There is a one-dimensional space of $P$-invariant energies that arises from the standard energy on the circle, but we show surprisingly that there are higher dimensional spaces of energies invariant under iterates of $P$. There are two natural measures associated with the dynamics of $P$ on $\mathcal{J}$, the equilibrium measure $\mu$, which is $P$-invariant but ignores the geometric aspects of the $P$ action, and the conformal measure $\nu$, which is not $P$-invariant but does transform according to a power of the Jacobian of the mapping. The $P$-invariant Laplacian $\Delta_{\mu}$ is built from the $P$-invariant energy and the measure $\mu$. This Laplacian will depend only on the topological type of $\mathcal{J}$ (so for quasicircles, it just gives the usual Laplacian on the circle). The conformal Laplacian $\Delta_{\nu}$ is built from the $P$-invariant energy and the measure $\nu$.

We describe numerical procedures to approximate the eigenvalues and eigenfunctions of the Laplacians $\Delta_{\mu}$ and $\Delta_{\nu}$ and present the computational results. For $\Delta_{\mu}$ we identify a 4 -element $\left(\mathbf{Z}_{2} \oplus \mathbf{Z}_{2}\right)$ group of symmetries. In the case of the basilica the symmetries are generated by horizontal and vertical reflections, but in the case of the rabbit and other Julia sets the symmetries are more hidden (only $z \rightarrow-z$ is obvious). Based on these symmetries we are able to classify eigenfunctions and explain the computational data.
\end{abstract}

\section{INTRODUCTION}

Analysis on fractals has been widely developed (see [Bar98, Kig01, Str06] and the references therein for a sample). The approach pioneered by Kigami has at its core a Laplacian that is built from energy and measure. Most of the examples studied so far have been linear fractals, such as the Sierpinski gasket, Sierpinski carpet, and Vicsek set. Recently [RT09] extended Kigami's approach to the basilica Julia sets. The Julia set $\mathcal{J}_{c}$ for the quadratic polynomials $P(z)=z^{2}+c$ with $c$ in the Mandelbrot set is a well studied class of nonlinear fractals that are both

Received by the editors June 30, 2009 and, in revised form, March 29, 2010

2010 Mathematics Subject Classification. Primary 28A80.

The research of the first author was supported by the National Science Foundation through the Research Experiences for Undergraduates Program at Cornell.

The research of the second author was supported in part by the National Science Foundation, grant DMS-0652440.

(C)2012 American Mathematical Society Reverts to public domain 28 years from publication 
connected and finitely ramified (they may be disconnected by removing a finite number of points) and so fit nicely into the Kigami paradigm:

(i) Approximate the fractal by a sequence of graphs $\Gamma_{m}$ with vertices $V_{m}$ and edge relation $x_{\tilde{m}} y$. We want the vertices to be nested,

with the union

$$
V_{0} \subseteq V_{1} \subseteq V_{2} \subseteq \cdots
$$

$$
V_{*}=\bigcup_{m=0}^{\infty} V_{m},
$$

a dense subset of the fractal.

(ii) On each graph $\Gamma_{m}$ construct an energy

$$
\mathcal{E}_{m}(u, v)=\sum_{x_{\tilde{m}} y} c_{m}(x, y)(u(x)-u(y))(v(x)-v(y)) .
$$

The conductances $c_{m}(x, y)$ are positive and may be interpreted as reciprocals of resistances, where the graph is viewed as an electric network with resistors of resistance $1 / c_{m}(x, y)$ connecting the nodes $x, y$. For any function $u$ defined on $V_{m}$, its harmonic extension $\tilde{u}$ to $V_{m+1}$ is defined to be the extension that minimizes energy:

$$
\mathcal{E}_{m+1}(\tilde{u}, \tilde{u}) \leq \mathcal{E}_{m+1}(u, u),
$$

where $\tilde{u}$ restricted to $V_{m}$ equals $u$. We want to have the identity

$$
\mathcal{E}_{m+1}(\tilde{u}, \tilde{u})=\mathcal{E}_{m}(u, u) .
$$

It follows that for any function $u$ defined on $V_{*}$ we have increasing energy:

$$
\mathcal{E}_{0}(u, u) \leq \mathcal{E}_{1}(u, u) \leq \mathcal{E}_{2}(u, u) \leq \ldots,
$$

and so the definition

$$
\mathcal{E}(u, u)=\lim _{m \rightarrow \infty} \mathcal{E}_{m}(u, u)
$$

always makes sense. If we define the domain $\operatorname{dom} \mathcal{E}$ to be the functions with $\mathcal{E}(u, u)<\infty$, then

$$
\mathcal{E}(u, v)=\lim _{m \rightarrow \infty} \mathcal{E}_{m}(u, v)
$$

is always finite for $u, v \in \operatorname{dom} \mathcal{E}$.

(iii) We choose a measure $\mu$ on the fractal and define a Laplacian $\Delta_{\mu}$ by the weak formulation

$$
-\mathcal{E}(u, v)=\int\left(\Delta_{\mu} u\right) v d \mu \text { for } v \in \operatorname{dom} \mathcal{E} .
$$

There is also a pointwise formula for $\Delta_{\mu} u$ on $V_{*}$ as a limit of graph Laplacians

$$
\Delta_{\mu} u(x)=\lim _{m \rightarrow \infty} \frac{1}{\int \psi_{x}^{(n)}} \Delta_{m} u(x),
$$

where

$$
\Delta_{m} u(x)=\sum_{y_{\widetilde{m}} x} c_{m}(x, y)(u(y)-u(x))
$$

and $\psi_{x}^{(m)}$ is the harmonic extension of the function $y \rightarrow \delta_{x y}$ on $V_{m}$. 
In the context of p.c.f. self-similar fractals, the energy is taken to have a self-similar structure. In the context of Julia sets we want to relate the energy to the dynamics of the action of $P$ on $\mathcal{J}$. The simplest requirement is $P$-invariance:

$$
\mathcal{E}(u \circ P, v \circ P)=c_{1} \mathcal{E}(u, v)
$$

(implicit in this is the statement that $u \in \operatorname{dom} \mathcal{E}$ implies $u \circ P \in \operatorname{dom} \mathcal{E}$ ). A somewhat weaker requirement is

$$
\mathcal{E}\left(u \circ P^{(k)}, v \circ P^{(k)}\right)=c_{k} \mathcal{E}(u, v)
$$

for some fixed $k$. Obviously (1.12) implies (1.13) for all $k$. In the case of the unit circle $(c=0)$, all solutions of (1.13) are multiples of the standard energy

$$
\mathcal{E}(u, v)=\int_{0}^{2 \pi} u^{\prime}(\theta) v^{\prime}(\theta) d \theta
$$

and so (1.13) implies (1.12). We will see in section 3 that in general there are more solutions to (1.13) than to (1.12).

We use the method of external rays developed by Doaudy and Hubbard DH84 to parameterize the Julia set. Consider the Riemann mapping from the exterior of the unit circle to the exterior of $\mathcal{J}$ (normalized so that $\infty$ is mapped to $\infty$ and the point $z=1$ is mapped to the repelling fixed point of $P$ ). We denote by $\phi$ the restriction of the continuous extension of this mapping to the unit circle $C$ (this exists for $c$ in a hyperbolic component of the Mandlebrot set). For simplicity we parametrize the circle by $x=\frac{\theta}{2 \pi}$ in $[0,1]$. For the Julia sets we work with, $\phi$ is a continuous mapping onto $\mathcal{J}$, but typically it is not one-to-one (only in the case of quasicircles is it one-to-one). Thus $\mathcal{J}$ is realized as a quotient of $C$ by an infinite set of identifications of points. In section 2 we describe these identifications explicitly and give diagrams illustrating them. Since $\phi$ intertwines the action $P$ on $\mathcal{J}$ and $z \rightarrow z^{2}$ on $C$, the dynamics of $P$ are easily read from this parametrization. We also easily obtain a sequence of graphs $\Gamma_{m}$ on $\mathcal{J}$ (obtained after identifying points on graphs $\Gamma_{m}^{\prime}$ on $C$ ), where the vertices in $V_{m+1}$ are obtained from $V_{m}$ by applying $P^{-1}$.

As noted we require that $c$ is in a hyperbolic component of the Mandelbrot set. Equivalently we require that $P_{c}$ has an attracting fixed cycle. This cycle will be unique, so we may classify the Julia sets we work with by the period of this cycle, $k$. Note that $k$ will be constant on each hyperbolic component. The second requirement demands some notation. Call the connected component of $c$ in the filled in Julia set $U_{1}$. This is the region labeled 1 in Figure 1.1. We then require that the root point of $U_{1}$ be a fixed point of $P$. This implies the root has period $k$. Our last requirement is that the combinatorial rotation number of $c$ be \pm , i.e. that $P\left(U_{1}\right)$ is the next domain in the filled in Julia set connected to the root in the clockwise or counterclockwise direction. In the diagram below, this means we require that either $P\left(U_{1}\right)=U_{2}$ or $P\left(U_{2}\right)=U_{1}$. Given these restrictions, we take $c$ in the main cardioid $(k=1)$ or in a series of bulbs directly adjoining it, namely the $\frac{ \pm 1}{k}$-bulbs. See [Bra89] for further details.

In section 3 we study the energies $\mathcal{E}_{m}$ on the graphs $\Gamma_{m}$ that are naturally obtained from energies $\mathcal{E}_{m}^{\prime}$ on the graphs $\Gamma_{m}^{\prime}$ on $C$. The lengths of the intervals on $C$ between consecutive vertices are not all equal, but follow a pattern $\delta_{m}, 2 \delta_{m}, 4 \delta_{m}, \ldots, 2^{k-1} \delta_{m}$, where $k$ depends on the Julia set $(k=1$ for the circle, 

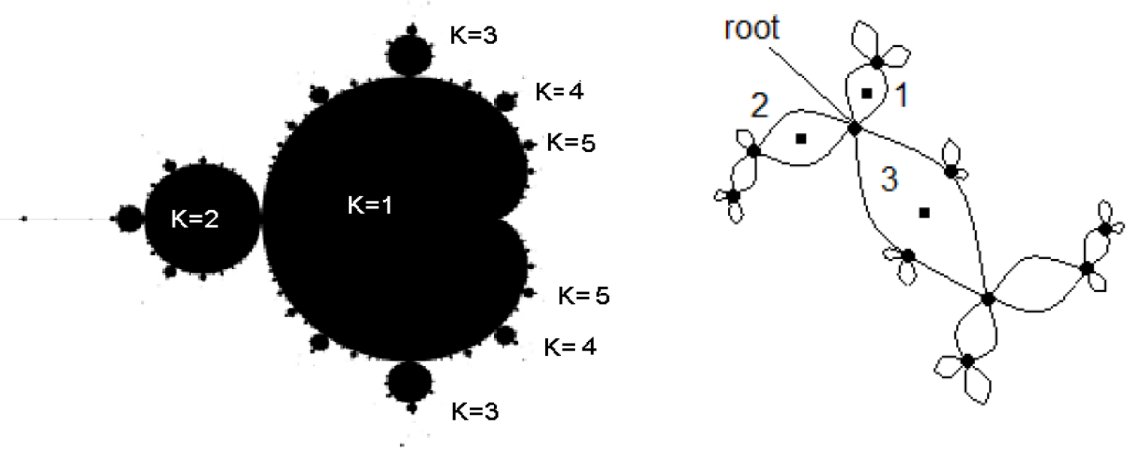

Figure 1.1. Left: Mandelbrot with the bulbs that satisfy our criterion marked by their $k$ value. Right: The rabbit with the root and the attracting cycle $\left(0, c, c^{2}+c\right)$ marked. The numbers reflect the dynamics of $P$ (region 1 maps to region 2 maps to region $3)$.

$k=2$ for the basilica, and $k=3$ for the rabbit) and $\delta_{m}=2^{-m} \delta$. For the standard energy on $C$, resistances are proportional to length, so on $\Gamma_{m}^{\prime}$

$$
E_{m}^{\prime}(u, v)=c \sum_{m} \sum_{j=0}^{k-1} 2^{m-j}\left(u\left(x_{n k+j+1}\right)-u\left(x_{n k+j}\right)\right)\left(v\left(x_{n k+j+1}\right)-v\left(x_{n k+j}\right)\right) .
$$

After identifying points (the vertices in $V_{m}^{\prime}$ have the property that all points identified with a vertex in $V_{m}^{\prime}$ are also in $V_{m}^{\prime}$ ) and multiplying by the renormalization factor $2^{\frac{1-k}{k} m}$, we obtain our energy $\mathcal{E}_{m}$ on $\Gamma_{m}$. However, it turns out that (1.5) does not hold (if $k \geq 2$ ). Instead we have

$$
\mathcal{E}_{m+k}(\tilde{u}, \tilde{u})=\mathcal{E}_{m}(u, u),
$$

where $\tilde{u}$ is the harmonic extension to $V_{m+k}$.

Thus we obtain $k$ different energies

$$
\mathcal{E}^{(j)}(u, v)=\lim _{m \rightarrow \infty} \mathcal{E}_{k m+j}(u, v) \text { for } j=0,1, \ldots, k-1 .
$$

It turns out that each of these energies is invariant under $P^{(k)}((1.13)$ holds), but

$$
\mathcal{E}^{(j)}(u \circ P, v \circ P)=2^{\frac{k+1}{k}} \mathcal{E}^{(j-1)}(u, v) .
$$

By taking the average

$$
\mathcal{E}(u, v)=\frac{1}{k} \sum_{j=0}^{k-1} \mathcal{E}^{(j)}(u, v)
$$

we obtain a $P$-invariant energy.

In section 4 we discuss the equilibrium measure $\mu$ and conformal measure $\nu$ on $\mathcal{J}$, and the Laplacians $\Delta_{\mu}$ and $\Delta_{\nu}$ constructed from the $P$-invariant energy (1.18) via (1.9) or (1.10). The measure $\mu$ is just the pullback to $\mathcal{J}$ of the normalized 
Lebesgue measure on the circle approximated on $V_{m}$ by the discrete measure that gives equal weight to all vertices and satisfies the $P$-invariance condition

$$
\int_{\mathcal{J}} f \circ P d \mu=\int_{\mathcal{J}} f d \mu .
$$

It follows that the Laplacian $\Delta_{\mu}$ also satisfies a $P$-invariance condition

$$
\Delta_{\mu}(u \circ P)=2^{1+\frac{1}{k}}\left(\Delta_{\mu} u\right) \circ P .
$$

In particular, if $u$ is a $\lambda$-eigenfunction,

$$
-\Delta_{\mu} u=\lambda u
$$

then $u \circ P$ is a $2^{\frac{1+k}{k}} \lambda$-eigenfunction. In other words, the spectrum of $\Delta_{\mu}$ is preserved under multiplication by $2^{1+1 / k}$.

Although the invariance condition (1.20) is very simple, it fails to take into account the geometry of the mapping $P$. The corresponding formula for Lebesgue measure in $\mathbf{R}^{n}$ would have a Jacobian factor. Since the mapping $P$ is conformal, the Jacobian is a power of $\left|P^{\prime}(z)\right|$. The conformal measure $\nu$ is characterized by the condition

$$
\int_{\mathcal{J}} f \circ P\left|P^{\prime}\right|^{d} d \nu=\int_{\mathcal{J}} f d \nu
$$

for a constant $d$ that may be identified as the Hausdorff dimension of $\mathcal{J}$. The Laplacian $\Delta_{\nu}$ depends on the geometry of $\mathcal{J}$, whereas $\Delta_{\mu}$ is the same for all Julia sets with the same topology. If we use the energies $\mathcal{E}^{(j)}$ instead of $\mathcal{E}$ we obtain Laplacians $\Delta_{\mu}^{(j)}$ and $\Delta_{\nu}^{(j)}$. We will show that the Laplacians $\Delta_{\mu}^{(j)}$ all commute, so they have the same eigenfunctions (with different eigenvalues).

In section 5 we discuss the symmetries of $\Delta_{\mu}$. Of course every Julia set has the symmetry $z \rightarrow-z$, and this is a symmetry of all the Laplacians. The circle has complete rotation symmetry. If we look at the basilica $(c=-1)$ we see that it is symmetric with respect to the vertical $R_{V}$ and the horizontal $R_{H}$ reflections. In the external ray parameterization these are also vertical and horizontal reflections, or $x \rightarrow-x$ and $x \rightarrow \frac{1}{2}-x$ (the product is $x \rightarrow \frac{1}{2}+x$, which corresponds to $z \rightarrow-z$ ). It is clear that these symmetries preserve all the energies and the measure $\mu$, but not the measure $\nu$. So $\Delta_{\mu}$ has a $\mathbf{Z}_{2} \oplus \mathbf{Z}_{2}$ symmetry group, and we can subdivide the eigenfunctions into four types according to even and odd behavior under $R_{V}$ and $R_{H}$. We write these as,,+++--+ , and -- . It is easy to see that an eigenfunction $u$ has the form $u=u^{\prime} \circ P$ for another eigenfunction $u^{\prime}$ if and only if it has the type ++ or -- . We call such an eigenfunction derived, and all others primitive. We show that reflectional symmetries of $\Delta_{\mu}$ exist on all the Julia sets we study. We continue to denote them by $R_{V}$ and $R_{H}$, although they are not simple vertical and horizontal reflections on the ambient plane and do not have the same description in the external ray parameterization.

In section 6 we describe the algorithms we use to compute approximation to the conformal measure $\nu$, the eigenfunctions, and eigenvalues of Laplacians. We present numerical data in graphical form for a few examples of quasicircles, the basilica, and the rabbit. Further computational results may be found in Flo08. We also show the eigenvalue counting functions and Weyl ratios. We have numerical evidence for eigenvalue gaps and spectral clusters. 
In section 7 we discuss the properties of the eigenfunctions of $\Delta_{\mu}$ that are visible in the data, using the symmetries of $\Delta_{\mu}$. In particular, we can predict the multiplicities of eigenspaces and the supports of eigenfunctions. This already goes beyond the results for the basilica obtained in [RT09].

Each Julia set can be viewed as the closure of a countable collection of topological circles that intersect at junction points (the junction points are dense in each circle) where exactly $k$ circles meet. In RT09 this structure is used to describe the energy. In section 8 we consider the restrictions of the eigenfunctions to one of these circles that we call the central circle. It corresponds to a Cantor set in the external ray parameterization. The portions of $\mathcal{J}$ that correspond to the complementary intervals of this Cantor set we will call loops. We will show that generically the restriction to a loop of any eigenfunction is determined by the eigenvalue, the size of the loop, and the value at the junction point. We will use this to produce an eigenfunction equation for a different Laplacian on the central circle.

Further development of the methods of this paper may be found in [ADS10. In particular, it treats a wider class of Julia sets, provides an alternate construction of the energy for our examples, and considers covering spaces and blowups of Julia sets.

\section{EXTERNAL RAY PARAMETERIZATION}

We start our graph approximation from a fixed point $z_{0}$ of $P$ lying in $\mathcal{J}$. For such a point we have $z^{2}+c=z$, which can be solved using the quadratic formula to produce $z_{ \pm}=\frac{1 \pm \sqrt{1-4 c}}{2} . z_{+}$will be a repelling fixed point (and thus in the Julia set) for all values of $c$. The same will be true of $z_{-}$for $c$ outside the main cardioid of the Mandlebrot set $(k \geq 2)$. We take $z_{+}=z_{0}$ when $k=1$ and $z_{-}=z_{0}$ otherwise. As this point is chosen such that $P\left(z_{0}\right)=z_{0}$, we have that $\phi\left(P\left(z_{0}\right)\right)=\phi\left(z_{0}\right)$ and thus that $2 x_{0}(\bmod 1)=x_{0}$, where $x_{0}=\phi\left(z_{0}\right)$. Given this we take $V_{0}^{\prime}=\left\{x: x=\phi\left(z_{0}\right)\right\}$, namely

$$
V_{0}^{\prime}=\left\{\frac{2^{j}}{2^{k}-1}\right\}, j=0,1, \ldots, k-1 .
$$

These points divide the circle into $k$ intervals

$$
\left[\frac{2^{j}}{2^{k}-1}, \frac{2^{j+1}}{2^{k}-1}\right]
$$

of lengths $\frac{2^{j}}{2^{k}-1}$ in the counterclockwise order. These points are all identified to give a single point in $V_{0}$.

We then define $V_{1}^{\prime}$ by $2 V_{1}^{\prime}=V_{0}$ (double cover). In other words, the points in $V_{1}^{\prime}$ have the form $\frac{1}{2} x$ and $\frac{1}{2} x+\frac{1}{2}$ as $x$ varies over $V_{0}^{\prime}$. Note that $V_{0}^{\prime} \subset V_{1}^{\prime}$. This is obvious for $\frac{2^{j}}{2^{k}-1}$ with $j \leq k-2$, but $\frac{2^{k-1}}{2^{k}-1} \frac{1}{2}=\left(\frac{1}{2^{k}-1}\right)+\frac{1}{2}$. The remaining $k$ points in $V_{1}^{\prime} \backslash V_{0}^{\prime}$ are $\frac{1}{2}\left(\frac{2^{j}}{2^{k}-1}\right)+\frac{1}{2}$ for $j=1, \ldots, k-1$ and $\frac{1}{2}\left(\frac{1}{2^{k}-1}\right)$. Note that they all lie in the interval $\left[\frac{2^{k-1}}{2^{k}-1}, \frac{1}{2^{k}-1}\right]$. The points in $V_{1}^{\prime}$ divide the circle into two cycles of intervals of length $\frac{1}{2} \frac{2^{j}}{2^{k}-1}$ for $j=0,1, \ldots, k-1$ in counterclockwise order. An equivalent description of the passage from $V_{0}^{\prime}$ to $V_{1}^{\prime}$ is the following: leave all the short intervals (of length $\frac{2^{j}}{2^{k}-1}$ for $j \leq k-2$ ) alone and subdivide the long intervals (of length $\frac{2^{k-1}}{2^{k}-1}$ ) into short intervals of lengths $\frac{2^{j}}{2^{k}-1}$ for $j \leq k-2$ and two of length 
$\frac{1}{2} \frac{1}{2^{k}-1}$. In passing from $V_{1}^{\prime}$ to $V_{1}$ we maintain the identification of points in $V_{0}$ and identify the $k$ new points in the long intervals. If we make $V_{1}^{\prime}$ into a graph $\Gamma_{1}^{\prime}$ by joining adjacent points on the circle by edges, then the induced graph $\Gamma_{1}$ has two edges joining its two vertices and two sets of $k-1$ self-edges at each point. These self-edges do not contribute to the energy, and so we will ignore them. The cases $k=2$ and $k=3$ are illustrated in Figure 2.1.
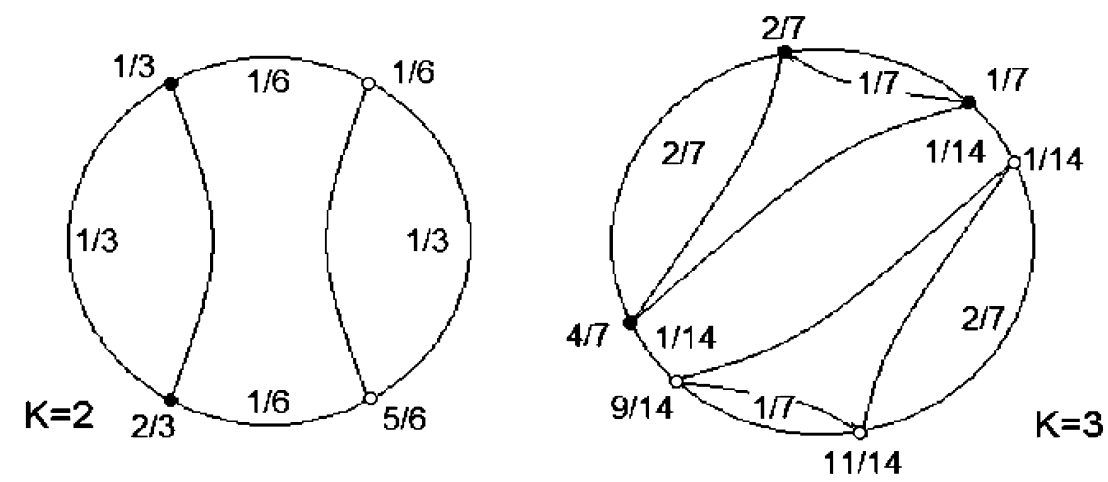

Figure 2.1. The vertices in $V_{0}$ in dark dots and in $V_{1} \backslash V_{0}$ in open dots. The lengths of the intervals are marked.

We can describe in similar terms the passage from $V_{m}^{\prime}$ to $V_{m+1}^{\prime}$. There are $2^{m} k$ points in $V_{m}^{\prime}$ that are identified to $2^{m}$ points in $V_{m}$. The points $V_{m}^{\prime}$ subdivide the circle into $2^{m}$ cycles of intervals of lengths $\frac{1}{2^{m}} \frac{2^{j}}{2^{k}-1}$ for $j=0, \ldots, k-1$ in counterclockwise order. The new points in $V_{m+1}^{\prime} \backslash V_{m}^{\prime}$ lie in the long intervals of length $\frac{1}{2^{m}} \frac{2^{k-1}}{2^{k}-1}$ and subdivide each such interval into short intervals of length $\frac{1}{2^{m}} \frac{2^{j}}{2^{k}-1}$ for $j=0, \ldots, k-1$ and two intervals of length $\frac{1}{2^{m+1}} \frac{1}{2^{k}-1}$ at the ends (note that $\left.\sum_{j=0}^{k-2} \frac{1}{2^{m}} \frac{2^{j}}{2^{k}-1}+2 \frac{1}{2^{m+1}} \frac{1}{2^{k}-1}=\frac{1}{2^{m}} \frac{2^{k-1}}{2^{k}-1}\right)$. The $k$ points in each long interval are identified when we pass from $V_{m+1}^{\prime}$ to $V_{m+1}$. Figure 2.2 shows several stages of subdivision for $k=2$ and Figure 2.3 does the same for $k=3$.
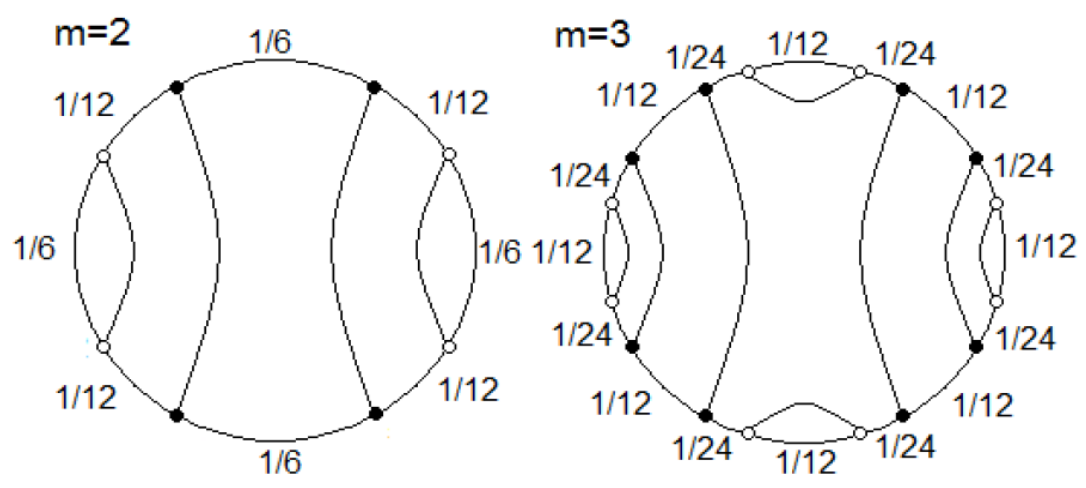

Figure 2.2. $k=2$. The vertices in $V_{m} \backslash V_{m-1}$ are labeled with open dots, and the lengths of the intervals are marked. 


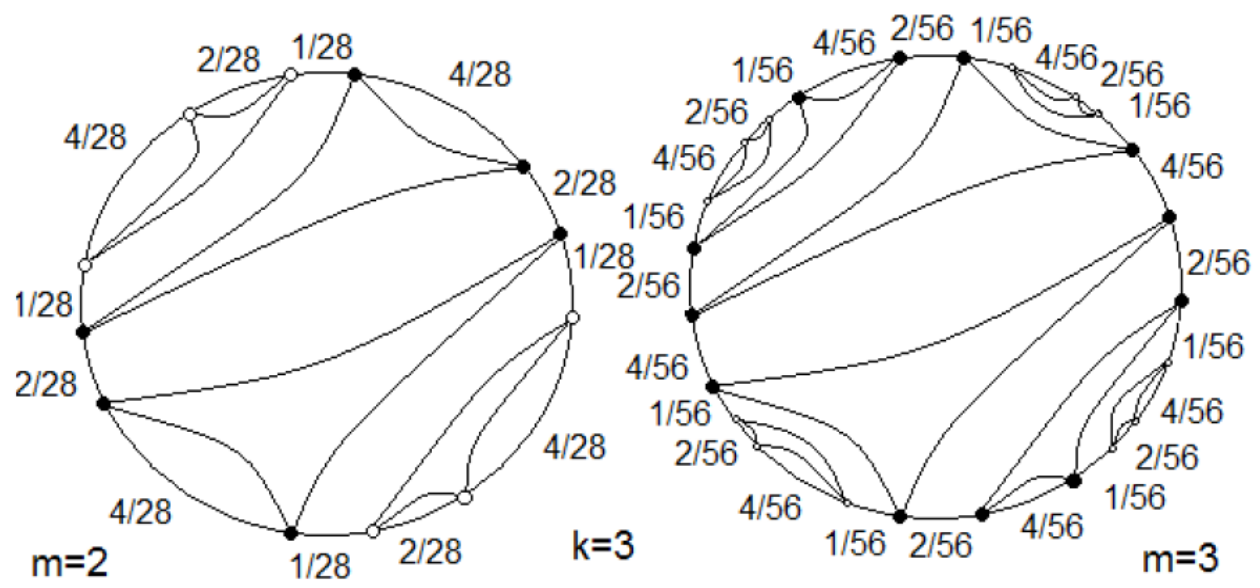

FiguRe 2.3

Next we describe the mapping $\phi$ from the circle to the Julia set in terms of the points in $V_{m}$. The point in $V_{0}$ is mapped to the fixed point of $P$ that is a junction point on the central circle. Then, inductively, the points in $V_{m+1}$ are mapped to the preimages of the points in $V_{m}$ under $P$. This is illustrated for the basilica $(k=2)$ and the rabbit $(k=3)$ in Figures 2.4 and 2.5. Note that the intervals in $V_{1}^{\prime}$ get mapped as follows: in each half the $k-1$ longest intervals that correspond to the self-edges get mapped to $k-1$ loops that join the central circle at either end, while the two short intervals get mapped to the central circle together with all the other loops that join it. These loops are images of intervals inside the short intervals, so the central circle is the image of their complement, a set of measure zero of Cantor type (it is not strictly speaking a Cantor set because it contains isolated points).

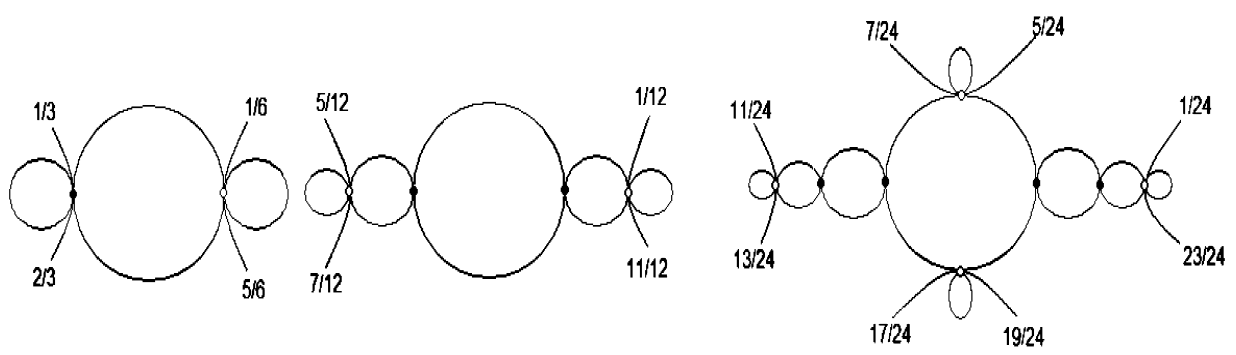

FiguRE 2.4 


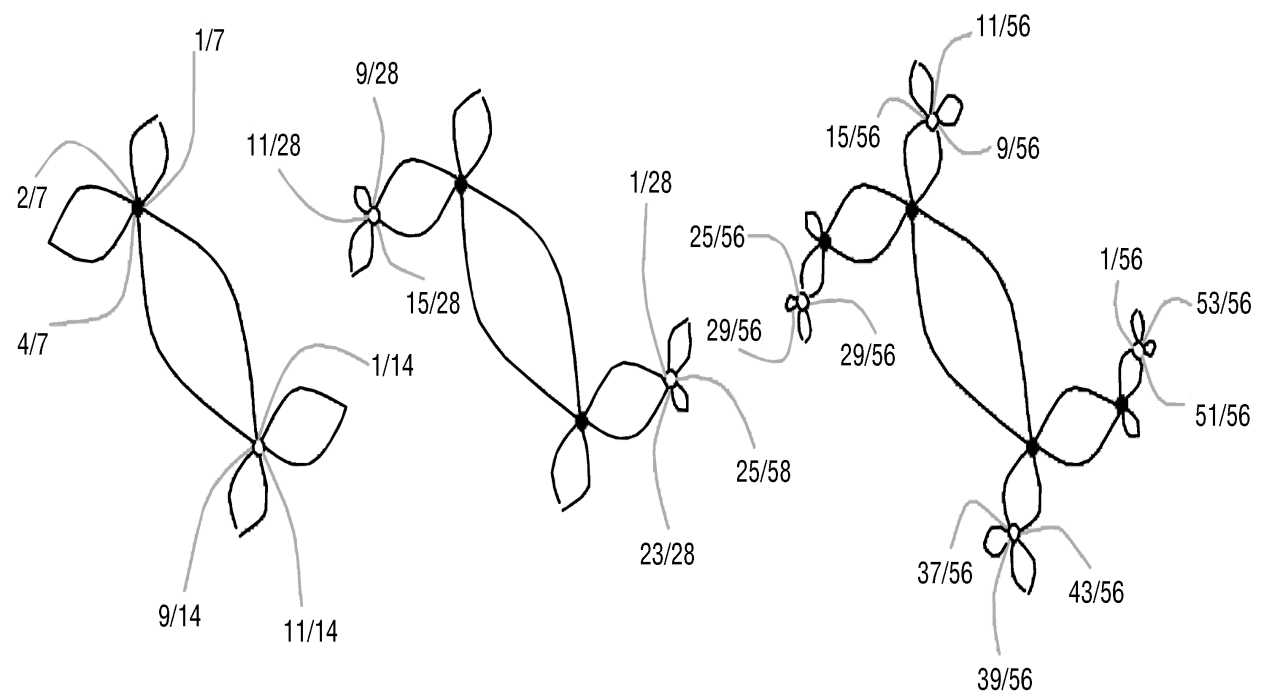

FiguRE 2.5

\section{ENERGIES}

The first step is to define energies $E_{m}$ on the graphs $\Gamma_{m}$. Consider the natural energy $E_{m}^{\prime}$ on $\Gamma_{m}^{\prime}$ defined by

$$
E_{m}^{\prime}(u, v)=\sum_{x_{\tilde{m}}^{\prime} y^{\prime}} \frac{1}{\left|x^{\prime}-y^{\prime}\right|}\left(u\left(x^{\prime}\right)-u\left(y^{\prime}\right)\right)\left(v\left(x^{\prime}\right)-v\left(y^{\prime}\right)\right) \quad\left(x^{\prime}, y^{\prime} \in V_{m}^{\prime}\right) .
$$

In other words, we take a resistance $\left|x^{\prime}-y^{\prime}\right|$ equal to the length of the interval connecting the consecutive vertices $x^{\prime}, y^{\prime}$. If $u$ and $v$ are defined on $V_{m}$, then we can regard them also as functions on $V_{m}^{\prime}$ which take the same value on identified points. Thus it makes sense to define

$$
E_{m}(u, v)=E_{m}^{\prime}(u, v) .
$$

In terms of the graph $\Gamma_{m}$, each vertex $x$ has $2 k$ edges of lengths $\frac{1}{2^{m}} \frac{2^{j}}{2^{k}-1}$ for $j=$ $0,1, \ldots, k-1$, each length repeated twice. Some of the edges may be self-edges, and some of the neighbors may repeat twice. Thus $E_{m}(u, v)$ has the form

$$
E_{m}(u, v)=\sum_{x_{\tilde{m}} y} c_{m}(x, y)(u(x)-u(y))(v(x)-v(y)) \quad\left(x, y \in V_{m}\right),
$$

where the conductance $c_{m}(x, y)$ is the sum of $\frac{1}{\left|x^{\prime}-y^{\prime}\right|}$ for all pairs $\left(x^{\prime}, y^{\prime}\right)$ mapping to $(x, y)$ with $x^{\prime}, y^{\prime}$ consecutive vertices in $V_{m}^{\prime}$.

The action of $P$ on $\mathcal{J}$ intertwines the action $x \rightarrow 2 x$ on $C$, so we will continue to write $P(x)=2 x$. We want to compute $E_{m}(u \circ P, v \circ P)=E_{m}^{\prime}(u \circ P, v \circ P)$. The action of $P$ maps $V_{m}$ to $V_{m-1}$ in a two-to-one fashion and respects the identification of points. We have $E_{m}^{\prime}(u \circ P, v \circ P)=2^{2} E_{m-1}^{\prime}(u, v)$, one factor of 2 coming from the double covering and one factor of 2 arising from the doubling of the length of intervals under $P$. Thus

$$
E_{m}(u \circ P, v \circ P)=4 E_{m-1}(u, v) .
$$


We will also need the iterated version

$$
E_{m}\left(u \circ P^{(k)}\right)=4^{k} E_{m-k}(u, v) .
$$

Now suppose $u$ is defined on $V_{m}$, and we want to compute the harmonic extension $\tilde{u}$ to $V_{m+1}$. Each new point $x$ in $V_{m+1} \backslash V_{m}$ consists of $k$ identified points in a long interval of length $\frac{1}{2^{m}} \frac{2^{k-1}}{2^{k}-1}$ between points $y^{\prime}$ and $z^{\prime}$ in $V_{m}^{\prime}$. The contribution to $E_{m+1}(\tilde{u}, \tilde{u})$ involving $\tilde{u}(x)$ is then

$$
2^{m+1}\left(2^{k}-1\right)\left(\left(\tilde{u}(x)-u\left(y^{\prime}\right)\right)^{2}+\left(\tilde{u}(x)-u\left(z^{\prime}\right)\right)^{2}\right) .
$$

This is clearly minimized by setting

$$
\tilde{u}(x)=\frac{1}{2}\left(u\left(y^{\prime}\right)+u\left(z^{\prime}\right)\right)
$$

which makes (3.6) equal to

$$
2^{m}\left(2^{k}-1\right)\left(u\left(y^{\prime}\right)-u\left(z^{\prime}\right)\right)^{2} .
$$

If $y^{\prime}$ and $z^{\prime}$ are identified in $V_{m}$, this is just 0 . Otherwise, it replaces the contribution

$$
2^{m-k+1}\left(2^{k}-1\right)\left(u\left(y^{\prime}\right)-u\left(z^{\prime}\right)\right)^{2}
$$

to $E_{m}^{\prime}(u, u)$ associated to the adjacent points $y^{\prime}, z^{\prime} \in V_{m}^{\prime}$. Note that (3.8) is simply (3.9) multiplied by $2^{k-1}$. On the other hand, for adjacent points $y^{\prime}, z^{\prime}$ in $V_{m}^{\prime}$ that are separated by a short interval of length $\frac{1}{2^{m}} \frac{2^{j}}{2^{k}-1}$ for $j \leq k-2$, the contribution to $E_{m+1}^{\prime}$ is the same as for $E_{m}^{\prime}$. Thus we definitely do not have $E_{m+1}(\tilde{u}, \tilde{u})$ equal to a constant multiple of $E_{m}(u, u)$.

Lemma 3.1. For the harmonic extension $\tilde{u}$ of $u$ from $V_{m}$ to $V_{m+k}$, we have

$$
E_{m+k}(\tilde{u}, \tilde{u})=2^{k-1} E_{m}(u, u) .
$$

Proof. Consider any pair of adjacent points $y^{\prime}, z^{\prime}$ separated by an interval of length $\frac{1}{2^{m}} \frac{2^{j}}{2^{k}-1}$. There will be no new points added between them until the passage from $E_{m+k-j-1}$ to $E_{m+k-j}$. At that stage, the contribution associated to the interval will be multiplied by $2^{k-1}$. After that stage, points will be inserted between identified points, so the harmonic extension will be constant and contribute 0 to the energy. Thus every term in the expression (3.1) for $E_{m}^{\prime}(u, u)$ gets multiplied by $2^{k-1}$ yielding (3.10).

We thus make the definition

$$
\mathcal{E}_{m}(u, v)=2^{\left(\frac{1-k}{k}\right) m} E_{m}(u, v)
$$

in order to replace (3.10) with

$$
\mathcal{E}_{m+k}(\tilde{u}, \tilde{u})=\mathcal{E}(u, u)
$$

For each $j=0,1, \ldots, k-1$ we have (for $u$ defined on $V_{k}$ )

$$
\mathcal{E}_{j+k m}(u, u) \leq \mathcal{E}_{j+k(m+1)}(u, u),
$$

and so it makes sense to define

$$
\mathcal{E}^{(j)}(u, v)=\lim _{m \rightarrow \infty} \mathcal{E}_{j+k m}(u, v)
$$

on $\operatorname{dom} \mathcal{E}^{(j)}$ defined to be the set of functions $u$ for which $\mathcal{E}^{(j)}(u, u)<\infty$. In fact the above argument shows that

$$
E_{m}(u, u)<E_{m+1}(\tilde{u}, \tilde{u}) \leq 2^{k-1} E_{m}(u, u),
$$


and so

$$
\mathcal{E}_{m}(u, u) \leq 2^{\frac{k-1}{k}} \mathcal{E}_{m+1}(u, u) \leq 2^{k-1} \mathcal{E}_{m+k}(u, u) .
$$

This implies

$$
2^{-\frac{(k-1)^{2}}{k}} \mathcal{E}^{(j+1)}(u, u) \leq \mathcal{E}^{(j)}(u, u) \leq 2^{\frac{k-1}{k}} \mathcal{E}^{(j+1)}(u, u),
$$

so $\operatorname{dom} \mathcal{E}^{(j)}$ is independent of $j$. Thus we denote it by $\operatorname{dom} \mathcal{E}$. Note that since the constant in (3.11) is less than one, nonconstant functions in dom $\mathcal{E}$ will have $E_{m}^{\prime}(u, u)$ going to infinity, and so will have infinite energy when viewed as functions on the circle.

Theorem 3.2. A function in dom $\mathcal{E}$, when considered as a function on $C$, is Hölder continuous of order $\frac{1}{2 k}$, and

$$
\left|u\left(x^{\prime}\right)-u\left(y^{\prime}\right)\right| \leq c \mathcal{E}^{(j)}(u, u)^{\frac{1}{2}}\left|x^{\prime}-y^{\prime}\right|^{\frac{1}{2 k}} \quad(\text { for any } j) .
$$

Proof. It suffices to show that (3.18) holds when $x^{\prime}, y^{\prime}$ are adjacent points in $V_{m}^{\prime}$ for some $m$. In that case $\left|x^{\prime}-y^{\prime}\right|$ is comparable to $2^{-m}$. From the definition (3.1) we have

$$
\left|u\left(x^{\prime}\right)-u\left(y^{\prime}\right)\right|^{2} \leq\left|x^{\prime}-y^{\prime}\right| E_{m}^{\prime}(u, u) .
$$

From the definition (3.11) we have

$$
E_{m}^{\prime}(u, u)=2^{\left(1-\frac{1}{k}\right) m} \mathcal{E}_{m}(u, u) \leq c\left|x^{\prime}-y^{\prime}\right|^{\frac{1}{k}-1} \mathcal{E}_{m}(u, u) .
$$

Combining (3.19) and (3.20) we obtain

$$
\left|u\left(x^{\prime}\right)-u\left(y^{\prime}\right)\right|^{2} \leq c\left|x^{\prime}-y^{\prime}\right|^{\frac{1}{k}} \mathcal{E}_{m}(u, u),
$$

which easily yields (3.18).

It follows that dom $\mathcal{E}$ embeds naturally in the continuous functions on $\mathcal{J}$, and $\operatorname{dom} \mathcal{E}$ modulo constants are a Hilbert space with inner product $\mathcal{E}^{(j)}$ for any $j$.

By combining (3.4) with the definition (3.11) we obtain

$$
\mathcal{E}_{m}(u \circ P, v \circ P)=2^{1+\frac{1}{k}} \mathcal{E}_{m-1}(u, v) .
$$

Theorem 3.3. If $u \in \operatorname{dom} \mathcal{E}$, then $u \circ P \in \operatorname{dom} \mathcal{E}$ and

$$
\mathcal{E}^{(j)}(u \circ P, v \circ P)=2^{1+\frac{1}{k}} \mathcal{E}^{(j-1)}(u, v) .
$$

It follows that

$$
\mathcal{E}^{(j)}\left(u \circ P^{(k)}, v \circ P^{(k)}\right)=2^{k+1} \mathcal{E}^{(j)}(u, v)
$$

so that there is a $k$-dimensional space of energies satisfying the invariance condition (3.24) under $P^{(k)}$. Moreover, if we define

$$
\mathcal{E}(u, v)=\frac{1}{k} \sum_{j=0}^{k-1} \mathcal{E}^{(j)}(u, v),
$$

then $\mathcal{E}$ satisfies the P-invariance condition

$$
\mathcal{E}(u \circ P, v \circ P)=2^{1+\frac{1}{k}} \mathcal{E}(u, v) .
$$

Proof. Replacing $m$ by $j+m k$ in (3.22) and passing to the limit we obtain (3.23). Iterating this $k$ times yields (3.24). It is not difficult to deduce from the discussion preceding Lemma 3.1 that all the energies $\mathcal{E}^{(j)}$ are distinct and linearly independent. Then (3.26) follows from (3.24) and (3.25). 


\section{LAPLACIANS}

In order to construct a Laplacian from the energy $\mathcal{E}$, we also need a measure. Since we want the Laplacian to reflect the dynamics of $P$ on $\mathcal{J}$, there are two natural choices. The simplest is the equilibrium measure $\mu$, which is just the pullback under $\phi: C \rightarrow \mathcal{J}$ of the normalized Lebesgue measure on $C$,

$$
\int_{\mathcal{J}} f d \mu=\int_{C} f \circ \phi d x
$$

For each interval $[a, b]$ in $C$, the image of $\phi([a, b])$ in $\mathcal{J}$ is assigned measure $b-a$. Since

$$
\int_{\mathcal{C}} f(2 x) d x=\int_{C} f(x) d x
$$

it follows that $\mu$ satisfies the $P$-invariance condition

$$
\int_{\mathcal{J}} f \circ P d \mu=\int_{\mathcal{J}} f d \mu .
$$

It is not difficult to see that (4.3) uniquely determines $\mu$ up to a constant multiple. Under any local one-to-one inverse of $P$, the measure is reduced by a factor of $\frac{1}{2}$.

It is easy to approximate $\mu$ by the discrete measure on $V_{m}^{\prime}$ that assigns equal weight $\frac{1}{2^{m} k}$ to each point so that on $V_{m}$ each point has weight $2^{-m}$. At first it appears that we could do a better job on $V_{m}$ by assigning weight to a point $x$ proportional to the length of the two intervals on either side of $x$, but in fact that would make no difference on $V_{m}^{\prime}$ since every point corresponds to $k$ points in $V_{m}$ with one representative of each weight.

If we look at a picture of $\mathcal{J}$ we see that $\mu$ assigns equal measure to loops that appear vastly different in size. If we want a measure that more accurately reflects the geometry of $\mathcal{J}$ in the Euclidean plane, we can use the conformal measure $\nu$ characterized by the identity

$$
\int_{\mathcal{J}}(f \circ P)\left|P^{\prime}\right|^{d} d \nu=\int_{\mathcal{J}} f d \nu
$$

where $d$ is the unique constant for which there exists a probability measure solution. It is known that $d$ is equal to the Hausdorff dimension of $\mathcal{J}$. Suppose $P: B \rightarrow A$ is one-to-one and $B$ is small enough that $P^{\prime}$ is approximately constant. Then by taking $f$ to be the characteristic function of $A$, (4.4) tells us that $\nu(A) \approx\left|P_{\mid B}^{\prime}\right|^{d} \nu(B)$. So when $\left|P^{\prime}\right|$ is larger the mapping $P$ multiplies the $\nu$ measure by a larger factor, and where it is smaller the factor is smaller. Since $\left|P^{\prime}(z)\right|=2|z|$ in all cases, the enlargement factor just depends on the distance to the origin. In fact, for $z$ inside the circle $|z|=\frac{1}{2}$, the measure is decreased.

To approximate $\nu$ by a discrete measure on $V_{m}$, we just have to assign weights $w_{m}(x)$ to all points $x \in V_{m}^{\prime}$. To have a probability measure we need

$$
\sum_{x \in V_{m}^{\prime}} w_{m}(x)=1
$$

To approximate (4.4) we want

$$
w_{m}(x) \approx|2 \phi(x)|^{d} w_{m-1}(2 x) .
$$


We can start with $w_{0}(x)=c$ for the points in $V_{0}^{\prime}$ that are identified with the unique point in $V_{0}$, and then use (4.6) to inductively define $w_{m}$ on $V_{m}$ (in terms of the parameter $d$ ). For large values of $d$

$$
\sum_{x \in V_{m}^{\prime}} w_{m}(x)
$$

will tend to 0 , and for small values of $d$ it will tend to $\infty$ as $m \rightarrow \infty$. The unique value $d$ is the one that lies in between, for which (4.7) remains bounded and has finite limit, and by adjusting the constant $c$ we can make the limit 1 . This gives us a slow trial and error algorithm to approximate both the dimension $d$ and the measure $\nu$.

Using the energy $\mathcal{E}$ and these measures, we construct the equilibrium Laplacian $\Delta_{\mu}$ and the conformal Laplacian $\Delta_{\nu}$ by (1.9) or (1.10). One could also construct Laplacians $\Delta_{\mu}^{(j)}$ and $\Delta_{\nu}^{(j)}$ using the energies $\mathcal{E}^{(j)}$ in place of $\mathcal{E}$.

The equilibrium Laplacian $\Delta_{\mu}$ satisfies the $P$-invariance condition

$$
\Delta_{\mu}(u \circ P)=2^{1+\frac{1}{k}}\left(\Delta_{\mu} u\right) \circ P
$$

as an immediate consequence of (4.3) and (3.26). The following important result is now obvious.

Theorem 4.1. If $u$ is an eigenfunction of $\Delta_{\mu}$ with eigenvalue $\lambda$, then $u \circ P^{(n)}$ is also an eigenfunction of $\Delta_{\mu}$ with eigenvalue $2^{n\left(1+\frac{1}{k}\right)} \lambda$. In particular, $2^{\left(1+\frac{1}{k}\right)} \Sigma_{\mu} \subseteq \Sigma_{\mu}$, where $\Sigma_{\mu}$ denotes the spectrum of $\Delta_{\mu}$.

Definition 4.2. An eigenfunction $u$ of $\Delta_{\mu}$ is called derived if there exists $u^{\prime}$ such that $u=u^{\prime} \circ P$ or primitive if no such $u^{\prime}$ exists. A derived eigenfunction is of class $D_{n}$ if $n$ is the largest integer such that $u=u^{\prime} \circ P^{(n)}$ for some eigenfunction $u^{\prime}$. A positive eigenvalue $\lambda \in \Sigma_{\mu}$ is called derived or primitive according to whether $2^{-\left(1+\frac{1}{k}\right)} \lambda$ is or is not in $\Sigma_{\mu}$, and a derived eigenvalue is of class $D_{n}$ if $n$ is the largest integer such that $2^{-n\left(1+\frac{1}{k}\right)} \lambda \in \Sigma_{\mu}$. Warning: the eigenspace of a derived eigenvalue may contain both derived and primitive eigenfunctions.

Theorem 4.3. An eigenfunction of $\Delta_{\mu}$ is derived if and only if it is even under $z \rightarrow-z$ :

$$
u(-z)=u(z) .
$$

Conversely, if an eigenfunction of $\Delta_{\mu}$ is odd,

$$
u(-z)=-u(z)
$$

then $u$ is primitive.

Proof. Suppose $u=u^{\prime} \circ P$ for some eigenfunction $u^{\prime}$. Then

$$
u(-z)=u^{\prime}(P(-z))=u^{\prime}(P(z))=u(z)
$$

since $P(-z)=P(z)$, so (4.9) holds for derived eigenfunctions. Since (4.10) is incompatible with (4.9), it implies that $u$ is primitive. Finally, suppose (4.9) holds. Define $u^{\prime}(z)=u\left(P^{-1} z\right)$. Note that (4.9) implies that $u^{\prime}$ is well defined, since both preimages of $z$ under $P$ differ by a minus sign. Then (4.8) shows that $u^{\prime}$ is an eigenfunction of $\Delta_{\mu}$, so $u$ is derived. 
If an eigenspace has multiplicity 1, then either (4.9) or (4.10) must hold, and this will distinguish the derived and the primitive. However, there are eigenspaces of higher multiplicity, and these split into direct sums of primitive and derived eigenfunctions using the usual even odd splitting of functions (obviously $z \rightarrow-z$ commutes with all Laplacians).

The equilibrium Laplacian $\Delta_{\mu}$ is defined entirely in terms of identifications on $C$ and the mapping $\phi$ from $C$ to $\mathcal{J}$, so it is essentially the same for all Julia sets with the same topology. For example, if $\mathcal{J}$ is a quasicircle, then $\Delta_{\mu}$ is just the ordinary Laplacian under the parameterization $\phi$. From this point of view, the conformal Laplacian is more interesting. However, the price we pay is that we cannot describe the structure of the spectrum of $\Delta_{\nu}$ in the same detail as for $\Delta_{\mu}$.

\section{Symmetries of THE EQUilibrium LAPLACIAN}

The rotation $z \rightarrow-z$ is an obvious symmetry of all Julia sets and all our Laplacians. In this section we describe two more symmetries of $\Delta_{\mu}$ that we will call vertical $R_{V}$ and horizontal $R_{H}$ reflections. In the case of the basilica $(k=2)$ these are exactly what they are, both on $\mathcal{J}$ and on $C$. In general they are different, but we will use the same terminology. The basic idea is that on the central circle we will take reflections and at junction points we will permute the loops that join.

First we describe $R_{H}$. In Figure 2.1 with $k=2$ a horizontal reflection maps vertices to vertices, and the same is true in Figure 2.2. However, in Figure 2.1 with $k=3$, if we reflect about the diameter through $\frac{3}{28}$ and $\frac{17}{28}$, we will permute $\frac{1}{14}$ and $\frac{1}{7}$ and also $\frac{4}{7}$ and $\frac{9}{14}$, but we will not permute $\frac{2}{7}$ and $\frac{11}{14}$. But the intervals $\left[\frac{1}{7}, \frac{2}{7}\right]$ and $\left[\frac{2}{7}, \frac{4}{7}\right]$ get mapped to two loops that join the central cycle at the same point. Since the order does not matter to the intrinsic geometry of $\mathcal{J}$, we can permute them by replacing $\frac{2}{7}$ by $\frac{3}{7}$, as shown in Figure 5.1 .

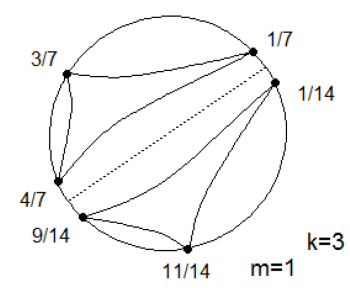

FIGURE 5.1
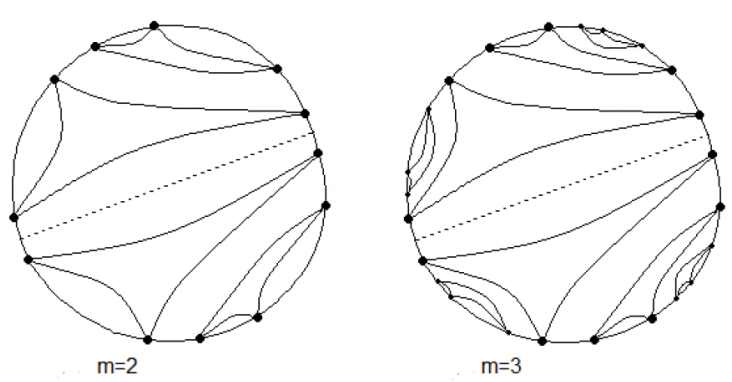

FiguRE 5.2

Now the reflection permutes all points. Of course we have to continue to permute the order of smaller loops to extend the reflection to the $V_{m}^{\prime}$ vertices shown in Figure 2.3 and now seen in Figure 5.2.

The same pattern persists for higher values of $m$ within the loops $\left[\frac{1}{7}, \frac{4}{7}\right]$ and $\left[\frac{9}{14}, \frac{1}{14}\right]$. These loops are isometric to each other if we do the appropriate permutation of subloops. However, something different has to be done in the interval, 
$\left[\frac{1}{14}, \frac{1}{7}\right]$ and $\left[\frac{4}{7}, \frac{9}{14}\right]$ that map to the central circle and the other loops that join it. At level $m=4$ we subdivide $\left[\frac{1}{14}, \frac{1}{7}\right]$, as shown in Figure 5.3.

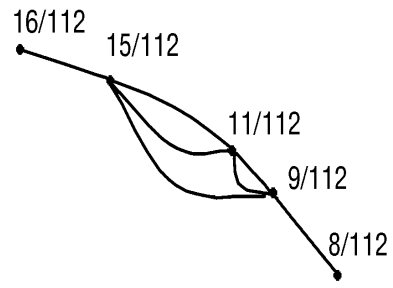

Figure 5.3

$R_{H}$ will permute the intervals $\left[\frac{8}{112}, \frac{9}{112}\right]$ and $\left[\frac{15}{112}, \frac{16}{112}\right]$ (with appropriate subloop swaps as these intervals subdivide for higher values of $m$ ), but it will preserve the loops $\left[\frac{9}{112}, \frac{11}{112}\right]$ and $\left[\frac{11}{112}, \frac{15}{112}\right]$, acting as a reflection on each one in a similar fashion: every time you subdivide a loop into two outer intervals and two inner loops, permute the outer intervals and preserve the inner loops.

To describe $R_{V}$ we want a modified reflection about the perpendicular diameter through $\frac{5}{14}$ and $\frac{12}{14}$. This will permute the intervals $\left[\frac{1}{14}, \frac{1}{7}\right]$ and $\left[\frac{4}{7}, \frac{9}{14}\right]$ with the appropriate swapping of subloops. However, the loops $\left[\frac{1}{7}, \frac{2}{7}\right],\left[\frac{2}{7}, \frac{4}{7}\right],\left[\frac{9}{14}, \frac{11}{14}\right]$ and $\left[\frac{11}{14}, \frac{15}{14}\right]$ are all preserved. When they are subdivided the rule is the same as for $R_{H}$ : the outer two intervals are permuted while the inner two loops are preserved.

It is not hard to see that $R_{H}$ and $R_{V}$ commute with $\Delta_{\mu}$, so eigenfunctions may be sorted according to whether they are even or odd. We write the $R_{V}$ symmetry first, so +- symmetry type means $u \circ R_{V}=+u$ and $u \circ R_{H}=-u$, etc. Functions of type ++ and -- satisfy (4.9), and functions of type +- and -+ satisfy (4.10). We call the +- type horizontal $(H)$ and the -+ type vertical $(V)$. By Theorem 4.3 we may sort the primitive eigenfunctions into $H$ and $V$ types. The key observation is that we may decompose $\mathcal{J}$ into horizontal (resp. vertical) segments joined at fixed points of $R_{H}$ (resp. $R_{V}$ ), such that the $H$ eigenfunctions behave independently on the segments: they can be multiplied by a different constant on each segment and remain eigenfunctions. In particular, if the eigenspace has multiplicity one, then the eigenfunction must be supported in one segment.

It is easy to see the segments on the basilica. The fixed points of $R_{V}$ are the junction points along the horizontal axis. In the $C$ parameter they are $\frac{1}{3} \sim \frac{2}{3}, \frac{1}{6} \sim$ $\frac{5}{6}, \frac{1}{12} \sim \frac{11}{12}, \frac{5}{12} \sim \frac{7}{12}, \ldots$ (in general $\frac{1}{3 \cdot 2^{m}} \sim 1-\frac{1}{3 \cdot 2^{m}}, \frac{1}{2}-\frac{1}{3 \cdot 2^{m}} \sim \frac{1}{2}+\frac{1}{3 \cdot 2^{m}}$ ), so the vertical segments are just the intersection of $\mathcal{J}$ with the vertical strips bounded by the vertical lines through the fixed points as shown in Figure 5.4.

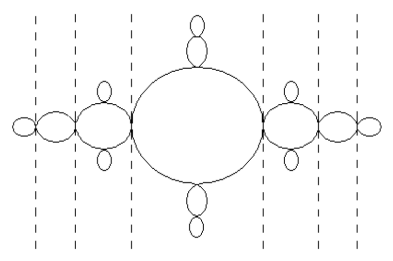

FiguRE 5.4 
Similarly, the fixed points of $R_{H}$ are the junction points along the vertical axis, with $C$ parameters $\frac{5}{24} \sim \frac{7}{24}, \frac{17}{24} \sim \frac{19}{24}, \frac{23}{96} \sim \frac{25}{96}, \frac{71}{96} \sim \frac{73}{96} \ldots$ (in general $\frac{1}{4}-\frac{1}{6 \cdot 4^{m}} \sim$ $\left.\frac{1}{4}+\frac{1}{6 \cdot 4^{m}}, \frac{3}{4}-\frac{1}{6 \cdot 4^{m}} \sim \frac{3}{4}+\frac{1}{6 \cdot 4^{m}}\right)$. The horizontal strips and segments are shown in Figure 5.5.

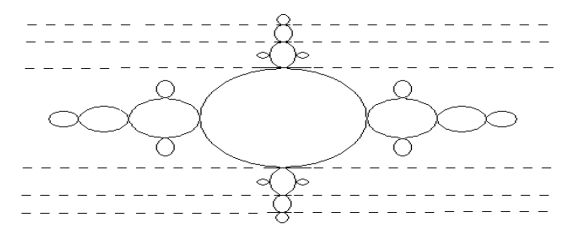

FigURE 5.5

The vertical segments are also clearly visible in the $C$ parameter space in Figure 2.2. Note that $R_{H}$ preserves the central vertical segment and permutes the other vertical segments in left-right pairs, while $R_{V}$ preserves all vertical segments. Similarly, $R_{V}$ preserves the central horizontal segment and permutes the other horizontal segments in up-down pairs, while $R_{H}$ preserves all horizontal segments.

In the general cases, the central vertical segment corresponds to the pair of intervals $\left[\frac{1}{2\left(2^{k}-1\right)}, \frac{1}{2^{k}-1}\right]$ and $\left[\frac{1}{2}+\frac{1}{2\left(2^{k}-1\right)}, \frac{1}{2}+\frac{1}{2^{k}-1}\right]$ between the points of $V_{1}^{\prime}$. Each time we subdivide a loop we add a vertical segment corresponding to the pair of small intervals at the ends of the loop. In terms of $\mathcal{J}$, a vertical segment contains a circle together with all loops that join it except at the midpoints. Similarly, a horizontal segment contains a circle together with all loops that join it except at the midpoints. In particular, the central horizontal segment in $C$ parameters is just the complement of the two intervals $\left[\frac{2^{k}+1}{2^{k+1}\left(2^{k}-1\right)}, \frac{2^{k+1}-1}{2^{k+1}\left(2^{k}-1\right)}\right]$ and $\left[\frac{1}{2}+\frac{2^{k}+1}{2^{k+1}\left(2^{k}-1\right)}, \frac{1}{2}+\right.$ $\frac{2^{k+1}-1}{2^{k+1}\left(2^{k}-1\right)}$. Again $R_{H}$ preserves the central vertical segment and permutes the other vertical segments in pairs, etc.

Theorem 5.1. Let $u$ be an eigenfunction of $\Delta_{\mu}$ that is odd with respect to $R_{H}$ (resp. $R_{V}$ ). If $\tilde{u}$ is the restriction of $u$ to any horizontal (resp. vertical) segment, defined to be equal to zero outside that segment, then $\tilde{u}$ is also an eigenfunction. In particular, if $\lambda$ is any primitive eigenvalue of multiplicity 1 , then the corresponding eigenfunction is supported on the central horizontal or vertical segment, depending on its $H$ or $V$ type.

Proof. The eigenvalue equation for $\tilde{u}$ is obvious at all points except the endpoints of the segment. Because $u$ is odd, $\tilde{u}$ vanishes at the endpoints. This also implies $\Delta_{\mu} \tilde{u}$ is zero at the endpoints. Thus the eigenvalue equation is trivially true at the endpoints $(0=\lambda 0)$. If the multiplicity of the eigenvalue is $1, u$ must vanish identically on all but one segment. If this were not a central segment, then we could obtain a linearly independent eigenfunction by composing $u$ with one of the reflections.

We will give more applications of this result in section 7 . 


\section{NumericAl DATA}

1. Conformal measure.

We present numerical results relevant to the computation of $\nu$. As Hausdorff dimension is used in (4.6) to derive $\nu$ its computation is given for each of the examples we will discuss: 2 quasicircles $(c=.2$ and $c=.33-.25 i)$, the standard basilica $(c=-1)$, one other $k=2$ type Julia set $(c=-1+.15 i)$ and the standard rabbit $(c=-.122+.745 i)$. To help build intuition we give the conformal measure, the corresponding cumulative distribution function, and $J^{\prime}(z)=2|z|$ on the standard basilica. For more computational results see [Flo08].

(i.) Computation of Hausdorff dimension $d$.

To approximate the Hausdorff dimension of the Julia set corresponding to $z^{2}+c$ we calculate the sum of weights assigned by equation (4.6) for $d$ chosen at regular intervals in $[1,2]$ on graph levels 1-22 (1-21 in the case of rabbit). If $d$ is chosen too large, the sequence will diverge, and for $d$ too small, the sequence tends to 0 . Given this we refine the grid. Continuing this process allows us to estimate $d$ to the desired accuracy. For $c=.2$ we estimate $d=1.0257$, using the sequence of graphs in Figure 6.1.

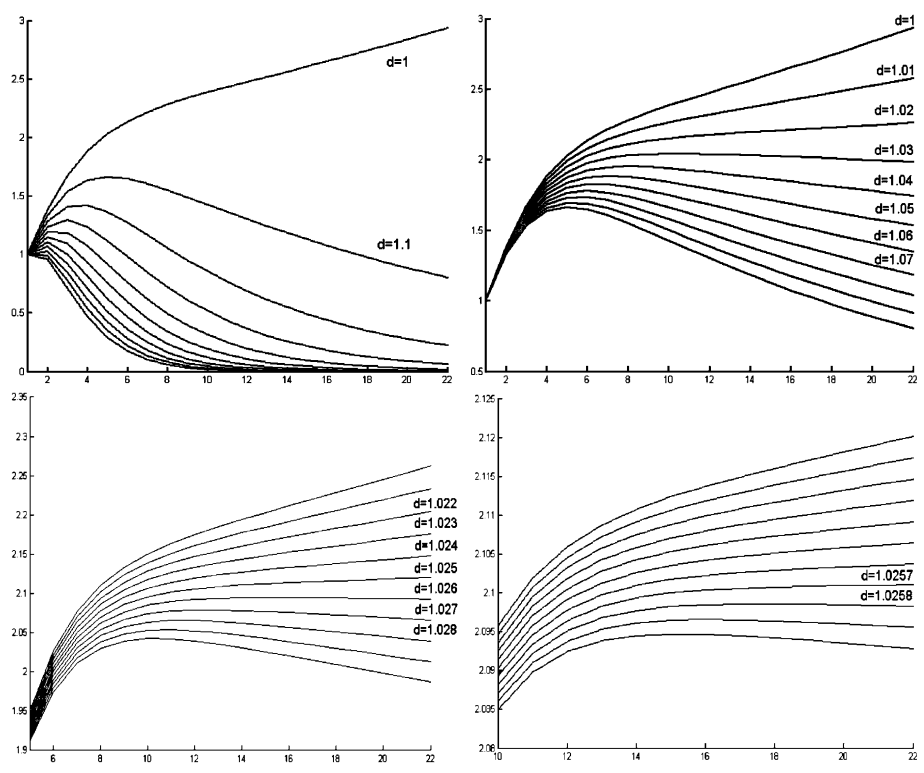

Figure 6.1. $c=.2$ Ranges of $d$ from top left to bottom right: $1-2$ in increments of .1, 1-1.1 by .01, $d$ ranges $1.02-1.03$ by $.001,1.025-1.026$ by .0001 .

For $c=.33-.25 i$ we estimate $d=1.1735$ in Figure 6.2.

For the basilica, $c=-1$, we approximate $d=1.2683$ in Figure 6.3.

Next we examine $c=-1+.15 i$ and estimate $d=1.3052$ in Figure 6.4.

Next we examine $c=-.122+.745 i$, the rabbit. We estimate $d=1.39$ in Figure 6.5. Because of the cycles in data, we would need greater computational power to improve this estimate. 


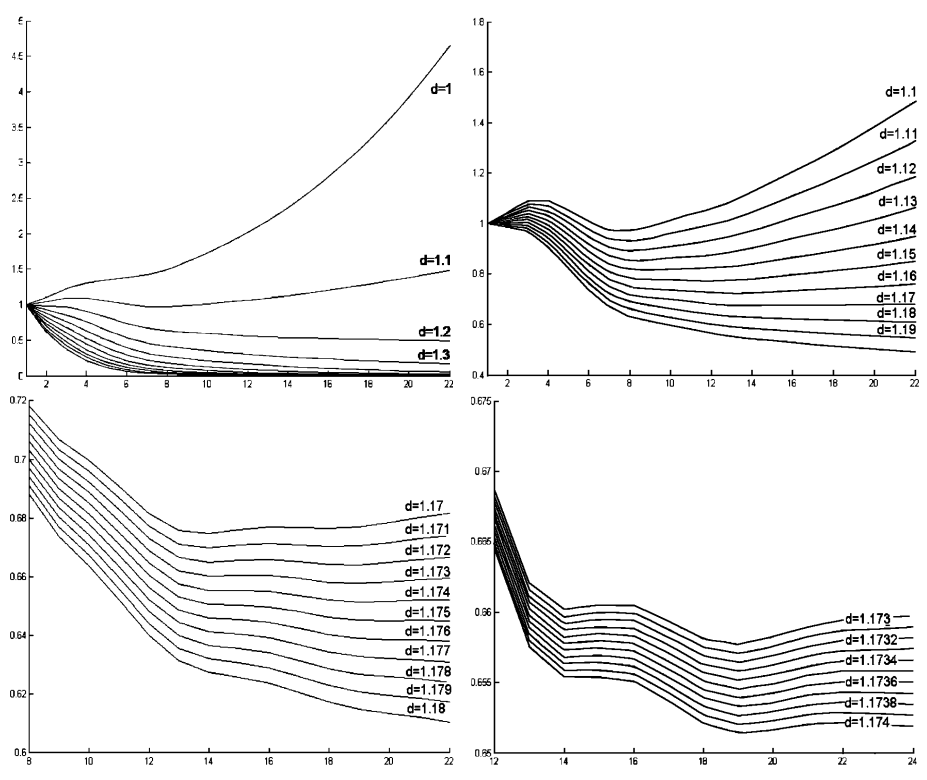

Figure 6.2. $c=.33-.25 i$ Ranges of $d$ from top left to bottom right: 1-2 in increments of .1, 1.1-1.2 in increments of .01, 1.17-1.18 in increments of .001, 1.173-1.174 in increments of .0001.

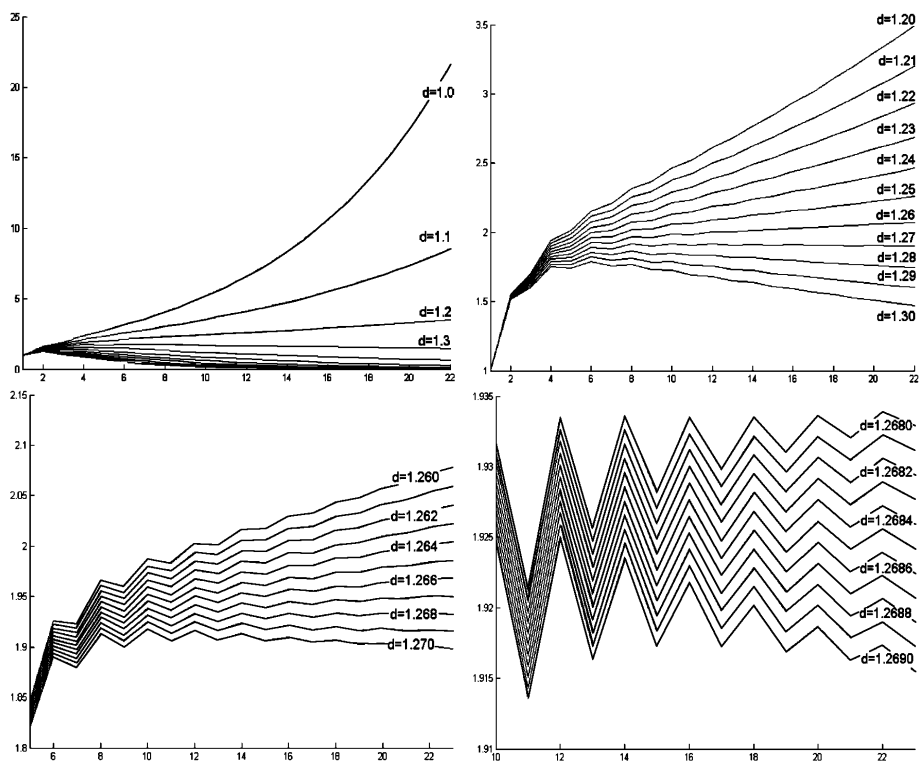

Figure 6.3. $c=.-1$ Ranges of $d$ from top left to bottom right: 1-2 in increments of .1, 1.2-1.3 in increments of .01, 1.261.27 in increments of .001, 1.268-1.269 in increments of .0001.

We note that these results correspond to our expectations. The Hausdorff dimension falls between 1 and 2 , and increases as we increase 


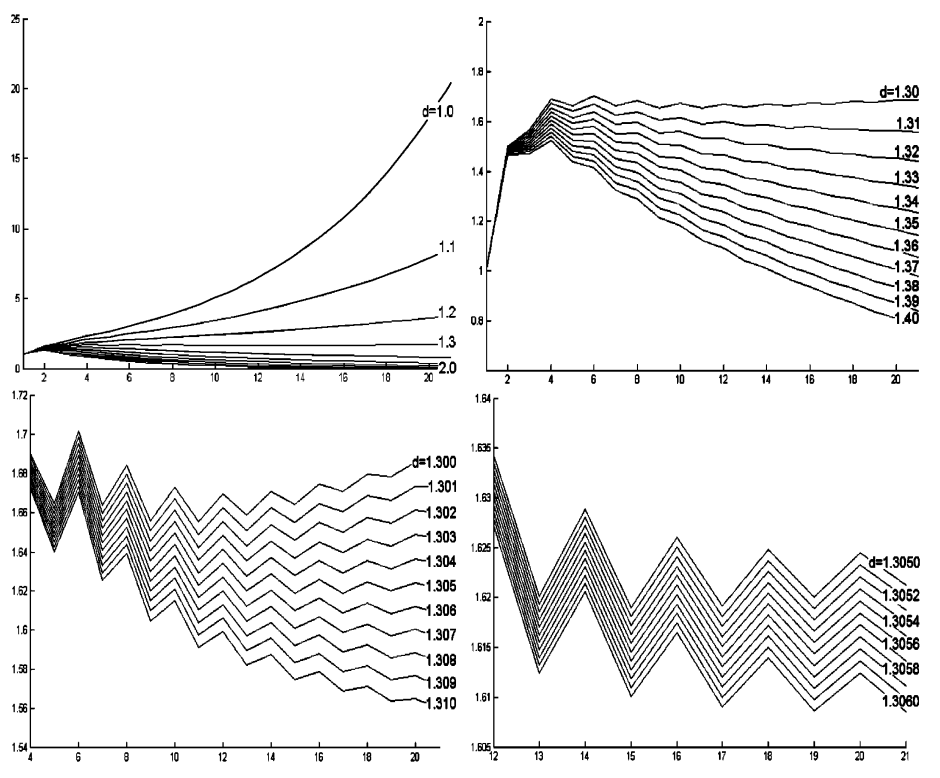

Figure 6.4. $c=-1+.15 i$ Ranges of $d$ from top left to bottom right: 1-2 in increments of .1, 1.3-1.4 in increments of .01, 1.30-1.31 in increments of .001, 1.305-1.306 in increments of .0001.

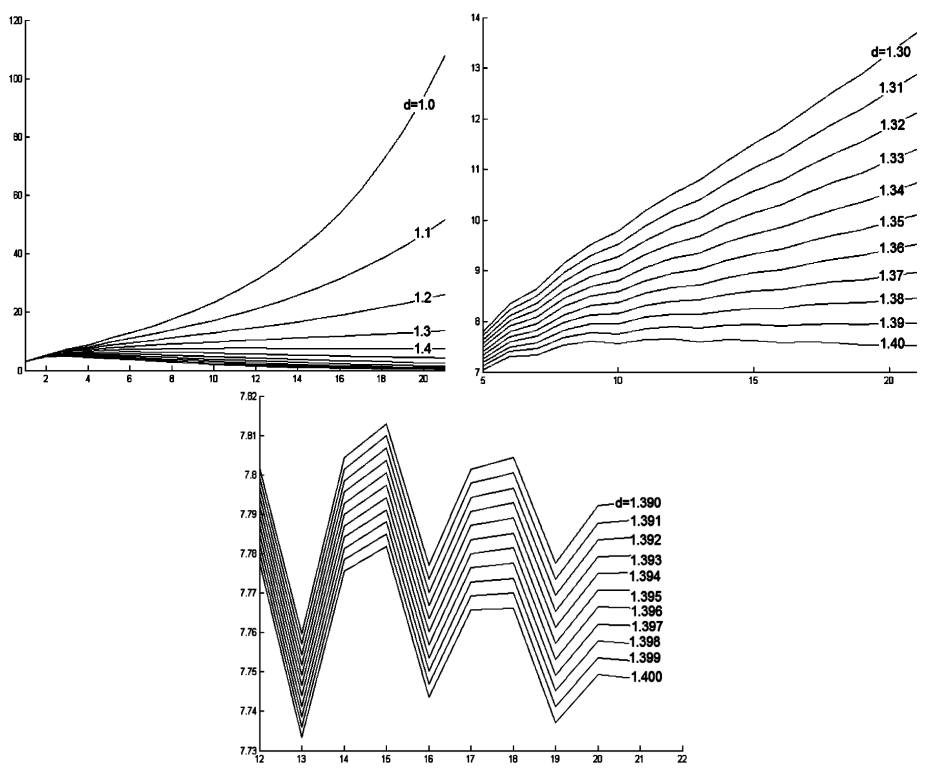

Figure 6.5. $c=-.122+.745 i$ Ranges of $d$ from left to right: 1-2 in increments of .1, 1.3-1.4 in increments of .01, 1.39-1.40 in increments of .001 . 
topological complexity, and for the $k=1$ and $k=2$ examples the set that intutitively looks more complex in fact has a higher estimated Hausdorff dimension.

(ii.) Data relevant to the computation of $\nu$ on the standard basilica, using $d=1.2683$.

Once we have an estimate of the Hausdorff dimension we use it to construct the conformal measure as in equation (4.6). This is pictured in Figure 6.6.
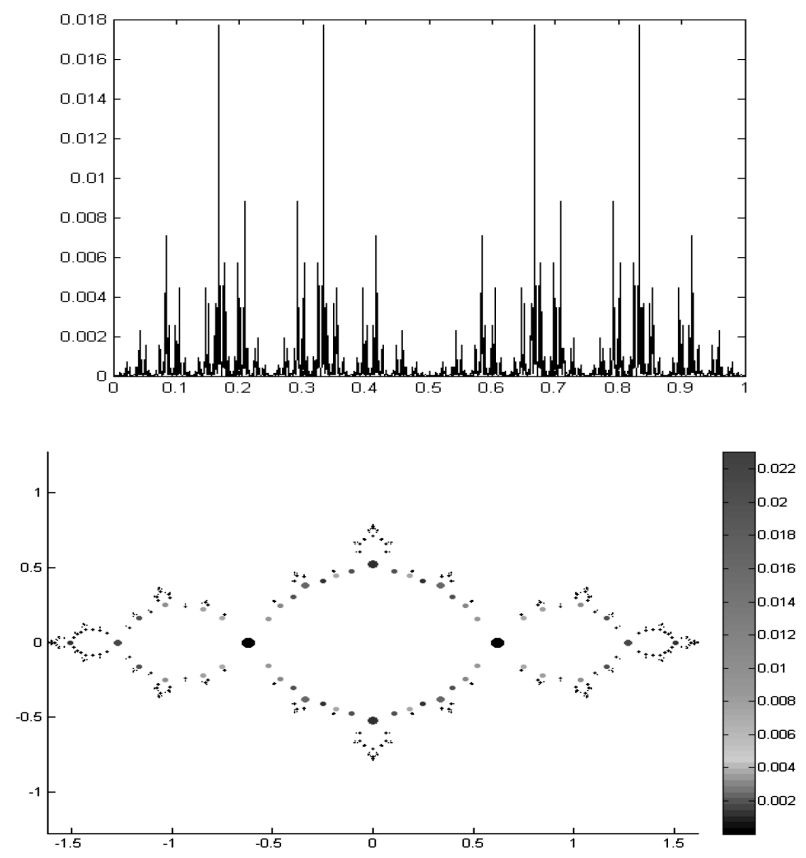

Figure 6.6. Top: $\nu$ with respect to ray parameterization. Bottom: approximation to $\nu$ by pointmasses.

We also give the cumulative distribution function for $\nu$ and the graph of $\left|J^{\prime}(z)\right|=2|z|$ as pointmasses (for comparsion to $\nu$ ). This is shown in Figure 6.7.

2. Spectra - Equilibrium measure.

(i.) Computation of the spectra.

Our program is divided into two pieces. First we set up the problem by computing the $m$-th level graph approximation in $C$ and in the ray parameter, as per the discussion in section 2 , recording the appropriate identifications. We then calculate weights given the choice of measure. Using this information we construct the $m$-th level Laplacian matrix as in (1.11). We then use Matlab(R2007b)'s Eig function to find the 

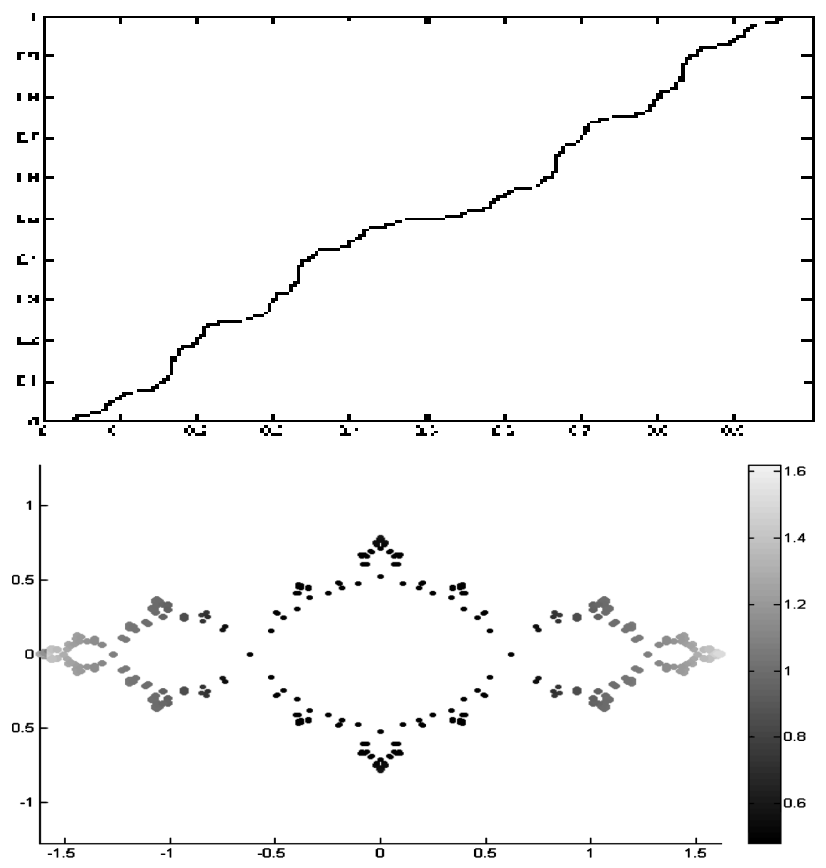

Figure 6.7. Top: $\nu([0, x])$. Bottom: approximation to $\left|J^{\prime}(z)\right|=$ $2|z|$ by pointmasses.

eigenvalues and eigenvectors of our Laplacian matrix. These results are given below.

(ii.) Computed eigenvalues and eigenfunctions for the basilica $c=-1$ ( $k=$ 2).

In Table 1 we give the computed eigenvalues on the last four computed levels of the graph $(10-13)$, a predicted value, $D_{n}\left(\right.$ Type $\left._{m}\right)$, and $2^{3 n / 2} *$ $\lambda_{\text {Type }_{m}}$. In Figure 6.9 there are pictures of the first 16 eigenfunctions as well as more detailed views of a few functions that show particularly interesting behavior.

We give the following for interpreting the tables. $D_{n}\left(\right.$ Type $\left._{m}\right)$, where Type is $V$ or $H$ depending on whether the function is vertical or horizontal, $m$ refers to the number of the primitive eigenfunction from which ours derives, and $n$ is the number of derivations required to reach the current eigenfunction. $2^{3 n / 2} * \lambda_{\text {Type }_{m}}$ is the eigenvalue predicted by the $P$-invariance condition on $\Delta_{\mu}$ given in (4.8). As the eigenvalue of $H 1$ and $V 1$ are known to have a high degree of accuracy, $2^{3 n / 2} * \lambda_{\text {Type }_{m}}$ will predict the eigenvalues of their derived eigenfunction with high accuracy. We then interpolate linearly between these values to obtain a prediction for arbitrary eigenfunctions. It should be noted that comparing eigenvalues predicted from the computation and by the equation $2^{3 n / 2} * \lambda_{\text {Type }_{m}}$ can alert us to approximation errors. 
TABLE 1. Computed actual eigenvalues of $\Delta_{\mu} c=-1$

\begin{tabular}{|c|c|c|c|c|c|c|c|}
\hline$\#$ & Level 10 & Level 11 & Level 12 & Level 13 & Predicted & $D_{n}\left(\right.$ Type $\left._{m}\right)$ & $2^{3 n / 2} * \lambda_{\text {Type }_{m}}$ \\
\hline 1 & 0 & 0 & 0 & 0 & - & - & - \\
\hline 2 & 39.868 & 39.869 & 39.870 & 39.870 & - & H1 & - \\
\hline 3 & 112.757 & 112.765 & 112.768 & 112.769 & 112.769 & D1(H1) & 112.769 \\
\hline 4 & 196.499 & 196.537 & 196.542 & 196.547 & 196.548 & V1 & - \\
\hline 5 & 274.985 & 275.045 & 275.060 & 275.068 & 275.071 & $\mathrm{H} 2$ & - \\
\hline 6 & 318.848 & 318.924 & 318.946 & 318.956 & 318.960 & D2(H1) & 318.960 \\
\hline $7-8$ & 555.662 & 555.784 & 555.890 & 555.905 & 555.918 & $\mathrm{D} 1(\mathrm{~V} 1)$ & 555.918 \\
\hline 9 & 777.435 & 777.774 & 777.946 & 777.988 & 778.012 & $\mathrm{D} 1(\mathrm{H} 2)$ & 778.010 \\
\hline 10 & 821.559 & 821.962 & 822.145 & 822.196 & 822.225 & H3 & - \\
\hline 11 & 901.338 & 901.838 & 902.054 & 902.116 & 902.154 & D3(H1) & 902.154 \\
\hline 12 & 1087.702 & 1088.651 & 1088.887 & 1089.006 & 1089.055 & $\mathrm{H} 4$ & - \\
\hline 13 & 1131.912 & 1132.953 & 1133.205 & 1133.335 & 1133.389 & $\mathrm{~V} 2$ & - \\
\hline $14-16$ & 1569.255 & 1571.648 & 1571.996 & 1572.294 & 1572.375 & $\mathrm{D} 2(\mathrm{~V} 1)$ & 1572.375 \\
\hline 17 & 2195.019 & 2198.918 & 2199.878 & 2200.364 & 2200.499 & $\mathrm{D} 2(\mathrm{H} 2)$ & 2200.544 \\
\hline 18 & 2242.639 & 2246.649 & 2247.673 & 2248.172 & 2248.333 & $\mathrm{H} 5$ & - \\
\hline 19 & 2319.552 & 2323.720 & 2324.859 & 2325.378 & 2325.568 & D1(H3) & 2325.521 \\
\hline 20 & 2430.056 & 2434.671 & 2435.908 & 2436.483 & 2436.705 & V3 & - \\
\hline 21 & 2501.764 & 2506.543 & 2507.895 & 2508.489 & 2508.744 & H6 & - \\
\hline 22 & 2544.471 & 2549.369 & 2550.784 & 2551.393 & 2551.678 & D4(H1) & 2551.678 \\
\hline 23 & 3069.346 & 3074.711 & 3077.393 & 3078.061 & 3078.503 & H7 & - \\
\hline 24 & 3071.110 & 3076.486 & 3079.169 & 3079.838 & 3080.381 & D1(H4) & 3080.173 \\
\hline $25-26$ & 3195.816 & 3201.531 & 3204.476 & 3205.187 & 3205.856 & $\mathrm{D} 1(\mathrm{~V} 2)$ & 3205.554 \\
\hline $27-32$ & 4430.630 & 4438.524 & 4445.292 & 4446.275 & 4447.347 & D3(V1) & 4447.347 \\
\hline 33 & 6186.513 & 6208.450 & 6219.478 & 6222.195 & 6223.763 & D3(H2) & 6224.079 \\
\hline 34 & 6229.699 & 6252.139 & 6263.256 & 6266.035 & 6267.683 & H8 & - \\
\hline 35 & 6319.742 & 6343.141 & 6354.483 & 6357.380 & 6359.121 & D1(H5) & 6358.792 \\
\hline 36 & 6377.333 & 6401.701 & 6413.067 & 6416.084 & 6417.911 & V4 & - \\
\hline 37 & 6480.064 & 6505.510 & 6517.154 & 6520.303 & 6522.231 & H9 & - \\
\hline 38 & 6534.651 & 6560.683 & 6572.474 & 6575.695 & 6577.712 & D2(H3) & 6577.565 \\
\hline $39-40$ & 6844.940 & 6873.236 & 6886.290 & 6889.789 & 6891.977 & D1(V3) & 6891.414 \\
\hline 41 & 7045.133 & 7076.056 & 7089.575 & 7093.397 & 7095.727 & D1(H6) & 7095.079 \\
\hline 42 & 7086.397 & 7117.912 & 7131.514 & 7135.409 & 7137.831 & H10 & - \\
\hline 43 & 7164.472 & 7196.852 & 7210.705 & 7214.706 & 7217.234 & D5(H1) & 7217.234 \\
\hline 44 & 7376.824 & 7413.193 & 7427.195 & 7431.687 & 7434.383 & H11 & - \\
\hline 45 & 7432.363 & 7469.456 & 7483.616 & 7488.196 & 7491.006 & V5 & - \\
\hline 46 & 8619.797 & 8681.423 & 8696.596 & 8704.182 & 8707.556 & $\mathrm{D} 1(\mathrm{H} 7)$ & 8706.070 \\
\hline 47 & 8622.301 & 8683.944 & 8699.133 & 8706.721 & 8710.204 & H12 & - \\
\hline 48 & 8624.752 & 8686.412 & 8701.615 & 8709.206 & 8712.799 & D2(H4) & 8712.045 \\
\hline $49-51$ & 8971.421 & 9039.132 & 9055.298 & 9063.627 & 9067.478 & $\mathrm{D} 2(\mathrm{~V} 2)$ & 9066.677 \\
\hline 52 & 11065.940 & 11185.447 & 11204.938 & 11219.571 & 11224.478 & V6 & - \\
\hline 53 & 11066.291 & 11185.804 & 11205.297 & 11219.931 & 11224.977 & H13 & - \\
\hline 54-64 & 12374.923 & 12531.714 & 12554.041 & 12573.185 & 12578.996 & $\mathrm{D} 4(\mathrm{~V} 1)$ & 12578.996 \\
\hline
\end{tabular}

Eigenvalue counting functions $N(x)$ and Weyl ratios $W(x)$ of $\Delta_{\mu}$ for $c=-1$ are illustrated in Figure 6.8. 


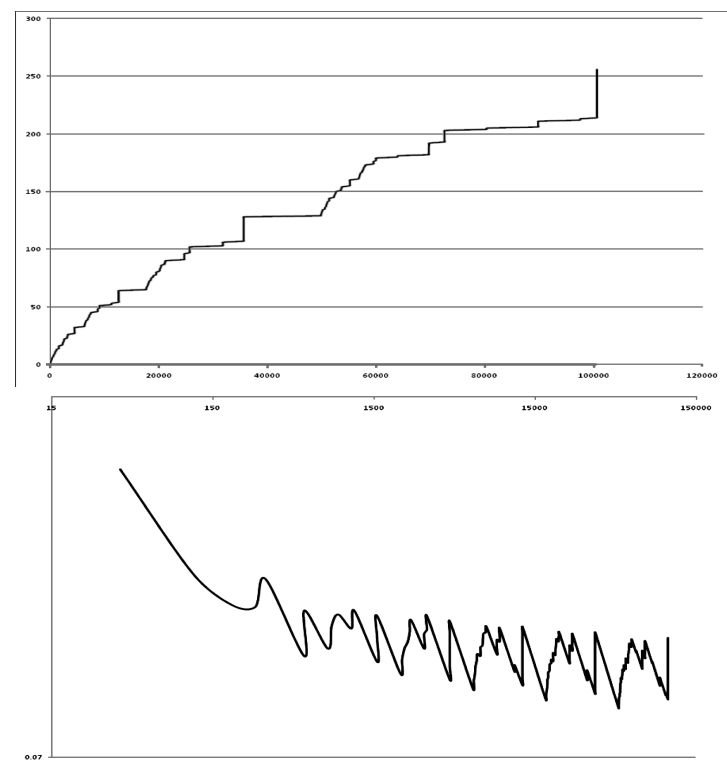

Figure 6.8. Top: Eigenvalue counting function and Bottom: Weyl ratio for predicted eigenvalues of $\Delta_{\mu}$ for $c=-1$. 

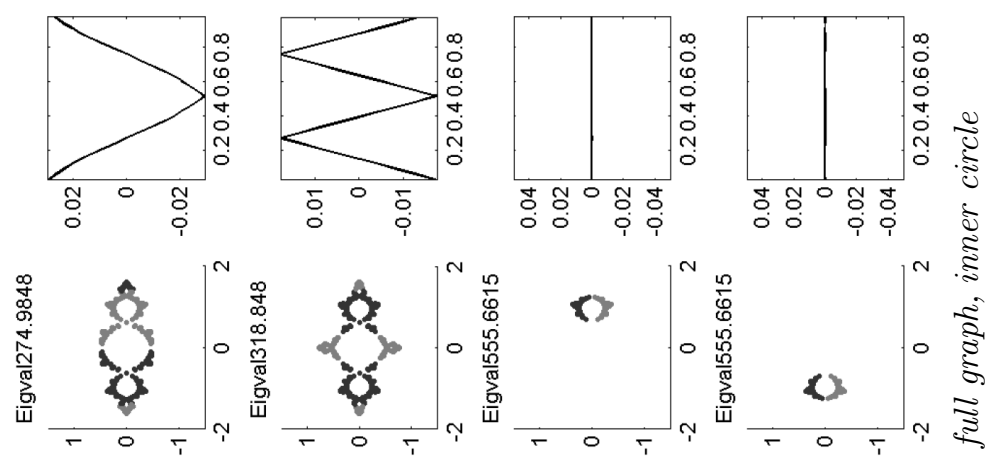

हี

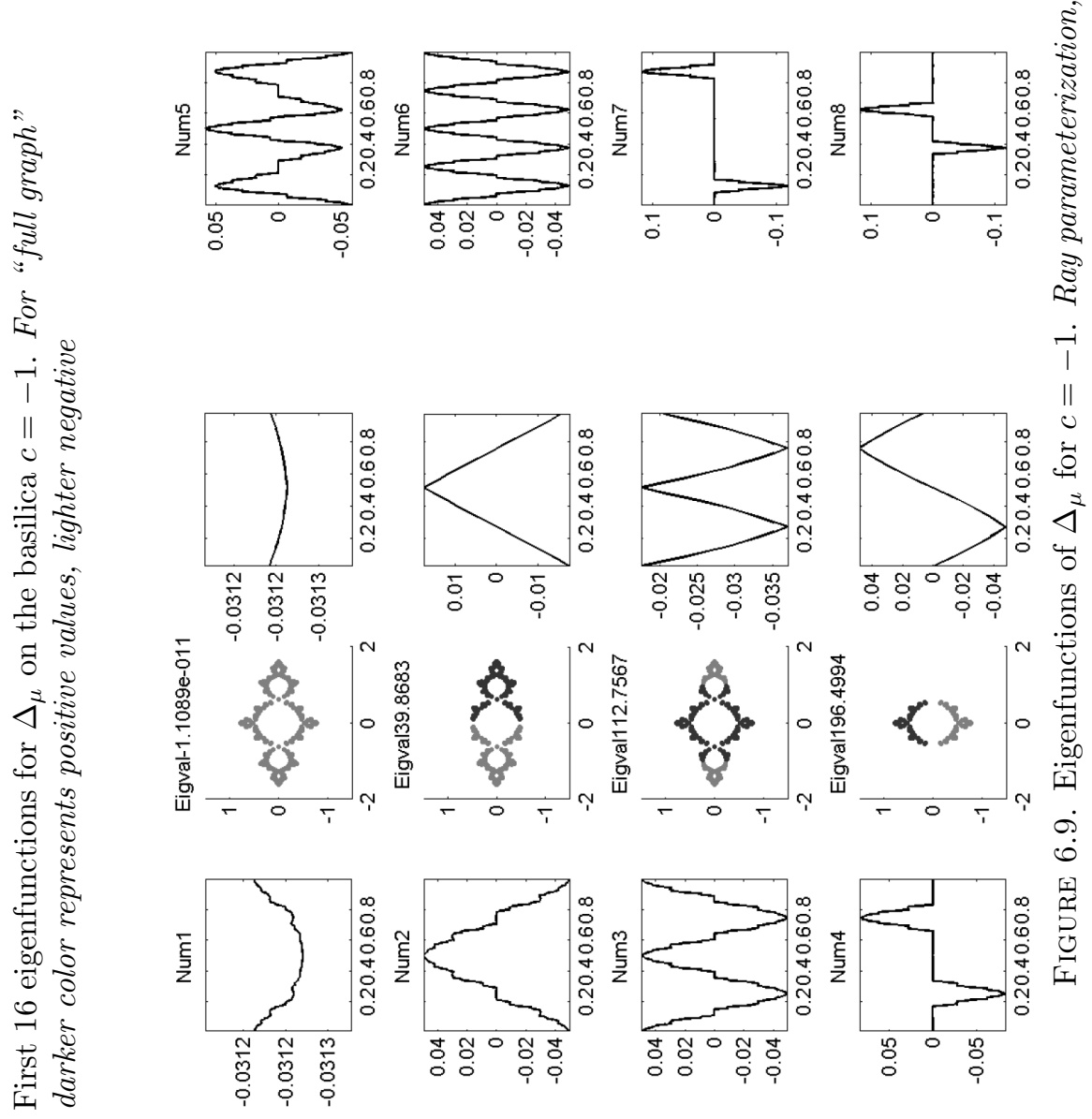



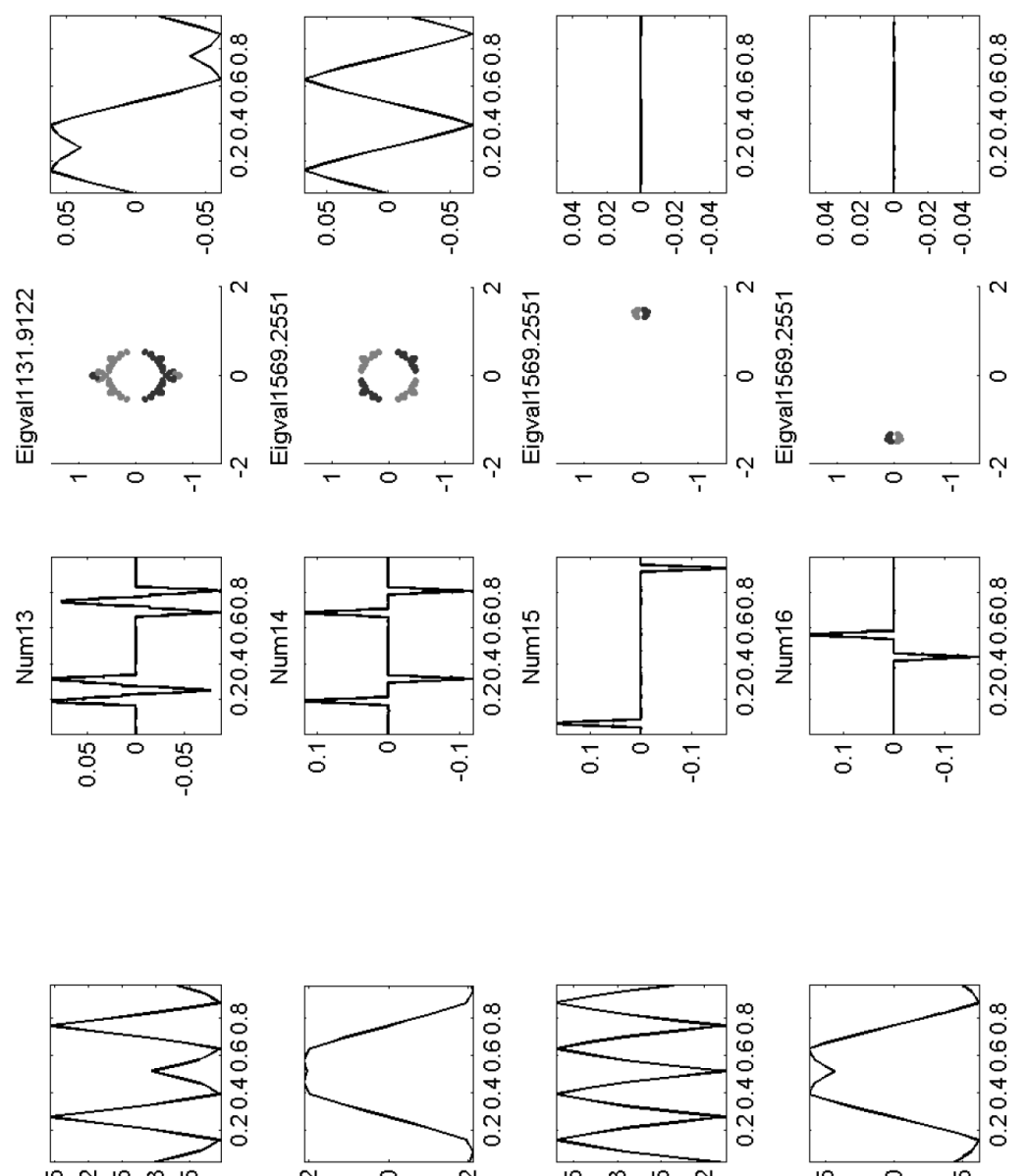

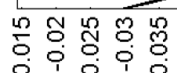
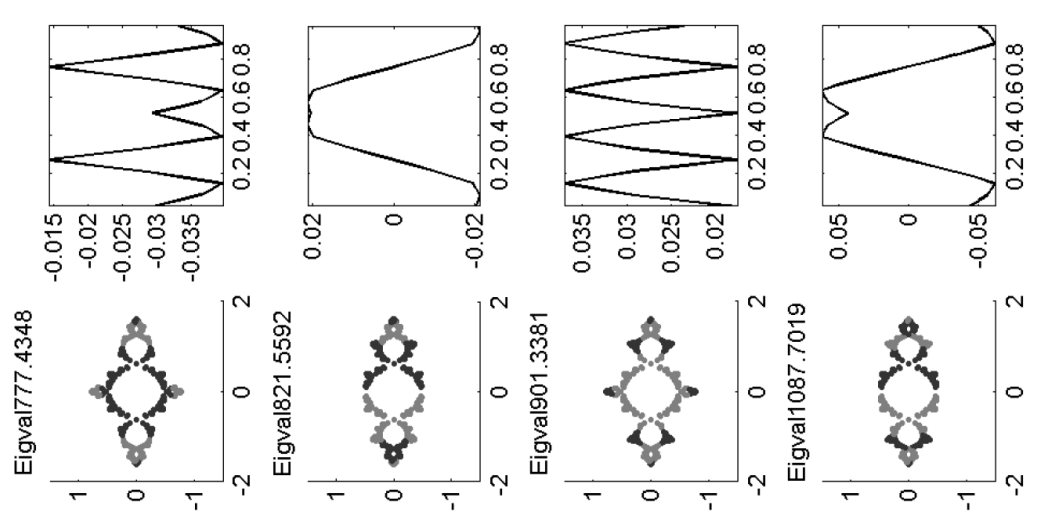

ปू.

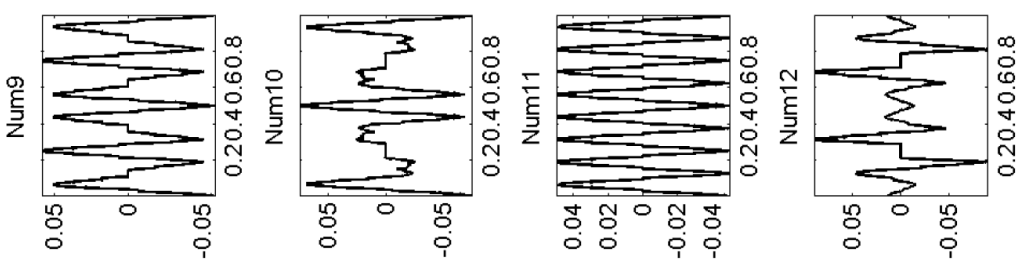


(iii.) Computed eigenvalues and eigenfunctions for the rabbit $c=-.122+$ $i .745(k=3)$.

For $c=-.122+i .745$ we give the computed eigenvalues on several levels of the graph, a predicted value, $D_{n}\left(\right.$ Type $\left._{m}\right)$, and $2^{4 n / 3} * \lambda_{\text {Type }_{m}}$. The labeling scheme in Table 2 is the same as that in the previous table. $2^{4 n / 3} * \lambda_{\text {Type }_{m}}$ is the eigenvalue predicted by the $P$-invarience condition on $\Delta_{\mu}$ given in (4.8). For Table 2, the predictions are based on the ratios of differences of successive terms, using the equation $=$ $V+(D) * R /(1-R)$ where $V$ is the last computed value and $D$ is the difference between $V$ and the value at the prior level. As the ratios between successive differences oscillate with period 3, when there is sufficient data (namely for the first 32 eigenfunctions) we take $R$ to be the average of the last 3 ratios. For $32-64$ we take $R$ to be the ratio of two.

TABLE 2. Computed actual eigenvalues of $\Delta_{\mu} c=-.122+$ $.745 i$

\begin{tabular}{|c|c|c|c|c|c|c|c|}
\hline \# & Level 8 & Level 9 & Level 10 & Level 11 & Predicted & $D_{n}\left(\right.$ Type $\left._{m}\right)$ & $2^{4 n / 3} * \lambda_{\text {Type }_{m}}$ \\
\hline 1 & 0.000 & 0.000 & 0.000 & 0.000 & 0.000 & - & - \\
\hline 2 & 165.721 & 165.824 & 165.883 & 165.904 & 165.923 & H1 & - \\
\hline 3 & 416.734 & 417.592 & 417.851 & 417.998 & 418.031 & D1(H1) & 418.052 \\
\hline 4 & 511.495 & 512.956 & 513.414 & 513.584 & 513.724 & H2 & - \\
\hline 5 & 1044.099 & 1050.103 & 1052.266 & 1052.919 & 1053.731 & D2(H1) & 1053.424 \\
\hline 6 & 1130.963 & 1137.403 & 1140.071 & 1140.884 & 1141.822 & H3 & - \\
\hline 7 & 1281.905 & 1288.888 & 1292.567 & 1293.721 & 1295.003 & $\mathrm{D} 1(\mathrm{H} 2)$ & 1294.150 \\
\hline 8 & 1714.862 & 1725.744 & 1733.302 & 1735.285 & 1738.810 & V1 & - \\
\hline 9 & 2461.466 & 2485.993 & 2499.697 & 2504.461 & 2508.700 & $\mathrm{H} 4$ & - \\
\hline 10 & 2603.427 & 2630.964 & 2646.093 & 2651.543 & 2655.987 & D3(H1) & 2654.462 \\
\hline 11 & 2805.593 & 2839.746 & 2855.919 & 2862.590 & 2866.231 & H5 & - \\
\hline 12 & 2815.301 & 2849.849 & 2866.075 & 2872.798 & 2876.407 & D1(H3) & 2874.848 \\
\hline 13 & 3151.277 & 3200.372 & 3217.576 & 3226.638 & 3228.738 & H6 & - \\
\hline 14 & 3180.544 & 3230.197 & 3247.793 & 3257.065 & 3259.212 & D2(H2) & 3261.054 \\
\hline $15-16$ & 4232.083 & 4321.182 & 4348.603 & 4367.648 & 4368.480 & D1(V1) & 4372.645 \\
\hline 17 & 5984.182 & 6202.505 & 6264.310 & 6298.841 & 6303.698 & D1(H4) & 6310.847 \\
\hline 18 & 6092.709 & 6321.151 & 6386.209 & 6421.640 & 6427.175 & $\mathrm{H} 7$ & - \\
\hline 19 & 6309.295 & 6560.226 & 6629.613 & 6667.737 & 6673.458 & $\mathrm{D} 4(\mathrm{H} 1)$ & 6688.825 \\
\hline 20 & 6421.773 & 6685.026 & 6758.788 & 6797.444 & 6804.497 & $\mathrm{H} 8$ & - \\
\hline 21 & 6764.448 & 7069.650 & 7155.712 & 7196.465 & 7207.290 & D1(H5) & 7213.276 \\
\hline 22 & 6773.988 & 7080.199 & 7166.696 & 7207.502 & 7218.460 & H9 & - \\
\hline 23 & 6786.602 & 7094.113 & 7181.169 & 7222.056 & 7233.172 & D2(H3) & 7244.163 \\
\hline 24 & 6988.374 & 7319.172 & 7416.793 & 7458.105 & 7473.183 & $\mathrm{~V} 2$ & - \\
\hline 25 & 7309.903 & 7685.383 & 7798.488 & 7840.769 & 7862.147 & H10 & - \\
\hline 26 & 7532.884 & 7940.721 & 8064.431 & 8107.784 & 8133.227 & D1(H6) & 8130.618 \\
\hline 27 & 7572.136 & 7986.284 & 8110.729 & 8154.747 & 8180.088 & H11 & - \\
\hline 28 & 7596.424 & 8014.467 & 8139.587 & 8183.926 & 8209.368 & D3(H2) & 8217.340 \\
\hline $29-32$ & 9795.501 & 10664.182 & 10888.697 & 10957.794 & 11022.945 & D2(V1) & 11018.375 \\
\hline 33 & 13391.179 & 15079.194 & 15629.334 & 15785.073 & 15870.193 & $\mathrm{D} 2(\mathrm{H} 4)$ & 15902.339 \\
\hline 34 & 13478.796 & 15187.726 & 15748.620 & 15907.468 & 15995.592 & H12 & - \\
\hline 35 & 13607.028 & 15352.665 & 15928.303 & 16092.240 & 16183.641 & $\mathrm{D} 1(\mathrm{H} 7)$ & 16181.519 \\
\hline 36 & 13687.105 & 15462.125 & 16050.142 & 16214.973 & 16309.161 & H13 & - \\
\hline 37 & 14014.754 & 15898.428 & 16530.733 & 16705.579 & 16809.660 & D5(H1) & 16854.782 \\
\hline 38 & 14082.089 & 15979.695 & 16621.093 & 16798.785 & 16905.929 & H14 & - \\
\hline 39 & 14250.885 & 16181.855 & 16845.211 & 17031.078 & 17145.843 & D1(H8) & 17128.485 \\
\hline 40 & 14708.995 & 16744.411 & 17478.016 & 17681.173 & 17821.144 & V3 & - \\
\hline
\end{tabular}


TABle 2. Computed actual eigenvalues of $\Delta_{\mu} c=-.122+$ $.745 i$

\begin{tabular}{cccccccc}
\hline$\#$ & Level 8 & Level 9 & Level 10 & Level 11 & Predicted & $D_{n}\left(\right.$ Type $\left._{m}\right)$ & $2^{4 n / 3} * \lambda_{\text {Type }_{m}}$ \\
\hline 41 & 14834.130 & 16889.618 & 17640.765 & 17850.884 & 17998.458 & $\mathrm{H} 15$ & - \\
42 & 14959.402 & 17045.341 & 17814.402 & 18031.263 & 18185.257 & $\mathrm{D} 2(\mathrm{H} 5)$ & 18176.317 \\
43 & 14977.379 & 17068.516 & 17840.036 & 18057.965 & 18212.708 & $\mathrm{H} 16$ & - \\
44 & 14977.979 & 17069.379 & 17840.983 & 18058.943 & 18213.698 & $\mathrm{D} 1(\mathrm{H} 9)$ & 18161.766 \\
45 & 14987.186 & 17082.284 & 17855.123 & 18073.616 & 18228.584 & $\mathrm{H} 17$ & - \\
46 & 15001.932 & 17101.165 & 17876.044 & 18095.412 & 18251.028 & $\mathrm{D} 3(\mathrm{H} 3)$ & 18254.146 \\
$47-48$ & 15416.746 & 17609.599 & 18443.159 & 18689.148 & 18868.023 & $\mathrm{D} 1(\mathrm{~V} 2)$ & 18793.248 \\
49 & 16085.687 & 18419.802 & 19365.950 & 19650.959 & 19882.827 & $\mathrm{D} 1(\mathrm{H} 10)$ & 19757.500 \\
50 & 16281.024 & 18665.713 & 19648.130 & 19944.139 & 20192.717 & $\mathrm{H} 18$ & - \\
51 & 16525.107 & 18981.677 & 20009.364 & 20321.092 & 20589.538 & $\mathrm{D} 2(\mathrm{H} 6)$ & 20487.873 \\
52 & 16574.303 & 19050.999 & 20089.820 & 20403.281 & 20675.662 & $\mathrm{H} 19$ & - \\
53 & 16592.597 & 19080.588 & 20124.174 & 20437.758 & 20711.110 & $\mathrm{D} 1(\mathrm{H} 11)$ & 20548.674 \\
54 & 16597.938 & 19088.831 & 20133.808 & 20447.430 & 20721.151 & $\mathrm{H} 20$ & - \\
55 & 16636.257 & 19141.788 & 20195.192 & 20510.474 & 20787.384 & $\mathrm{D} 4(\mathrm{H} 2)$ & 20706.400 \\
56 & 17991.470 & 20860.052 & 22227.106 & 22606.649 & 23054.707 & $\mathrm{H} 21$ & - \\
57 & 18220.720 & 21192.118 & 22619.110 & 23012.838 & 23486.608 & $\mathrm{~V} 4$ & - \\
$58-64$ & 20748.808 & 24683.116 & 26872.054 & 27437.798 & 28376.990 & $\mathrm{D} 3(\mathrm{~V} 1)$ & 27764.564
\end{tabular}

Eigenvalue counting functions $N(x)$ and Weyl ratios $W(x)$ of $\Delta_{\mu}$ for $c=-.122+754 i$ are illustrated in Figure 6.10.

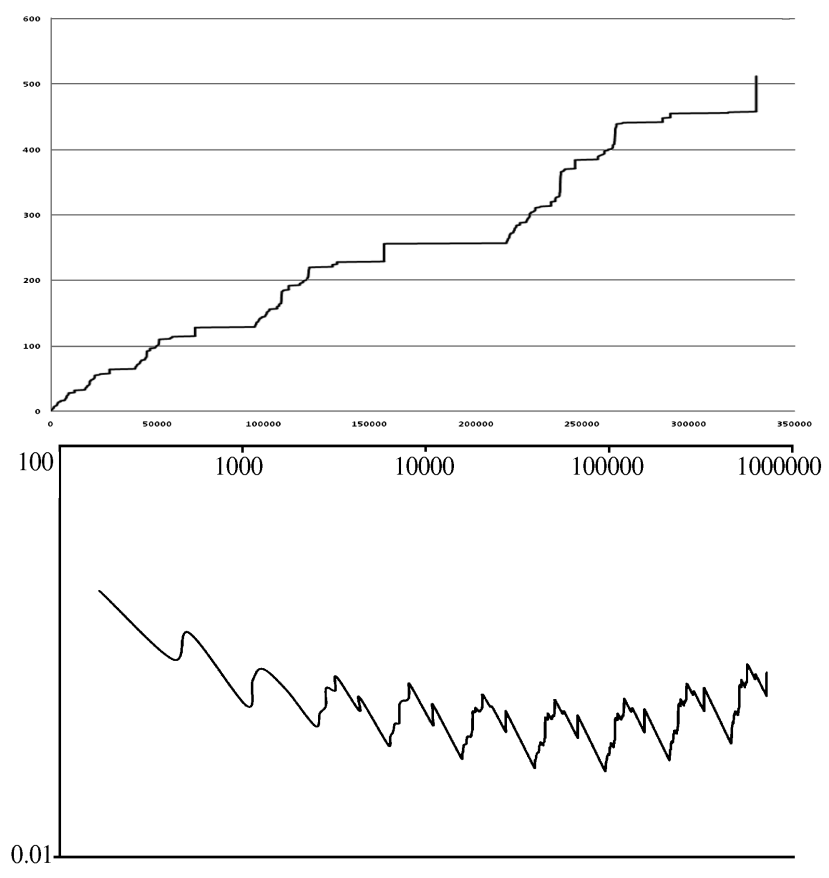

Figure 6.10. Top: Eigenvalue counting function. Bottom:

Weyl ratio for level 10 eigenvalues of $\Delta_{\mu}$ for $c=-.122+754 i$. 

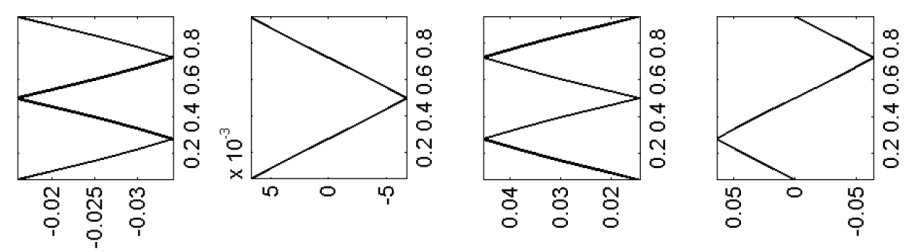

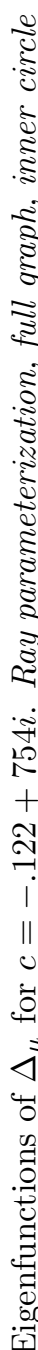

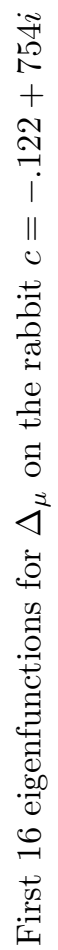
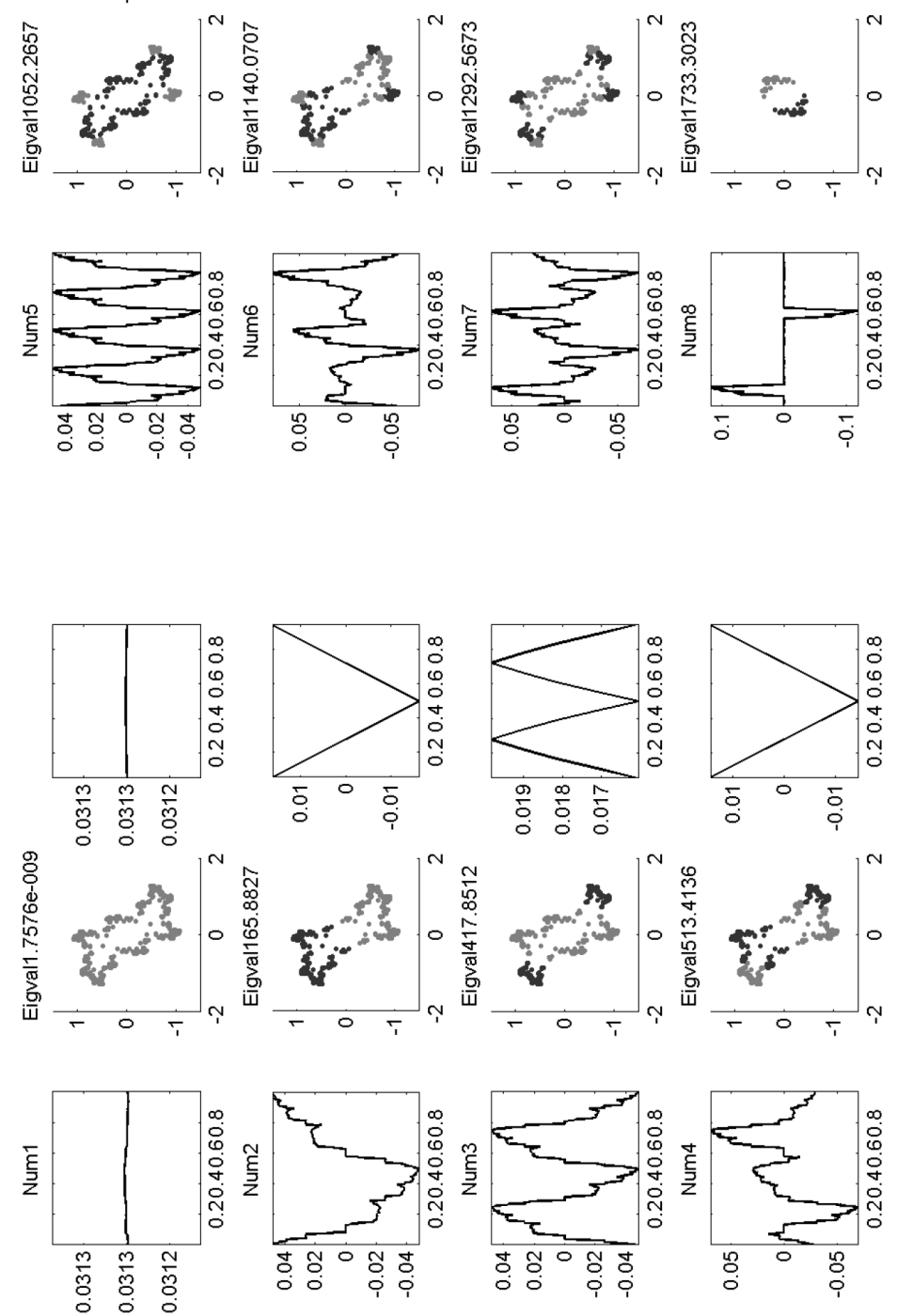

7 

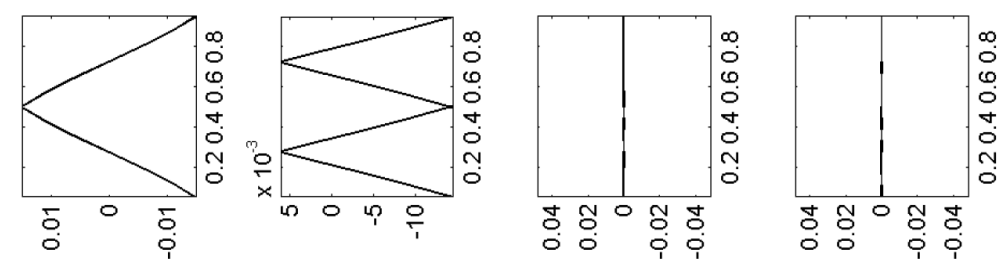

है
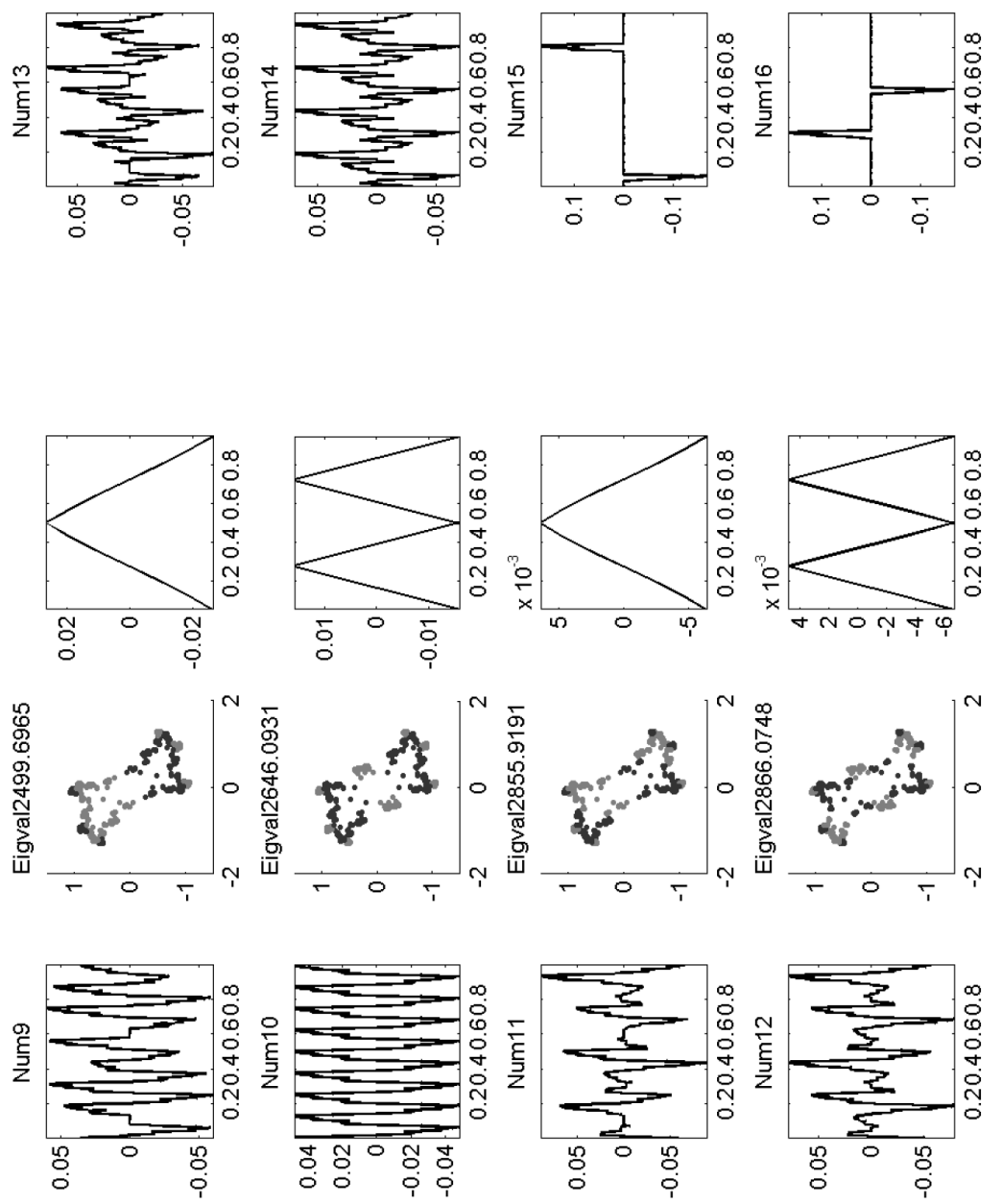
3. Computed eigenvalues for conformal measure.

(i.) Spectrum of $\Delta_{\nu}$ for $c=.33-i .25$.

TABLE 3. Computed actual eigenvalues of $\Delta_{\nu} c=.33-i .25$

\begin{tabular}{|c|c|c|c|c|}
\hline \# & Level 6 & Level 7 & Level 8 & Level 9 \\
\hline 1 & 0 & 0 & 0 & 0 \\
\hline 2 & 32.147045 & 32.493944 & 33.28344 & 33.567551 \\
\hline 3 & 48.213964 & 46.998041 & 45.831625 & 45.762296 \\
\hline 4 & 122.46591 & 117.836174 & 120.090215 & 124.059287 \\
\hline 5 & 221.515707 & 229.782687 & 217.016349 & 207.709143 \\
\hline 6 & 348.275565 & 349.237686 & 336.490519 & 334.507733 \\
\hline 7 & 402.528864 & 398.717183 & 382.160799 & 377.698993 \\
\hline 8 & 536.065552 & 513.365788 & 488.130227 & 493.410108 \\
\hline 9 & 876.420583 & 963.36787 & 974.702375 & 907.075854 \\
\hline 10 & 1038.939947 & 1081.255888 & 1074.177368 & 1015.481251 \\
\hline 11 & 1196.992559 & 1324.534496 & 1339.064483 & 1258.409975 \\
\hline 12 & 1432.349755 & 1505.609269 & 1499.557881 & 1415.362104 \\
\hline 13 & 71.287945 & 1844.610709 & 1820.091867 & 1725.159848 \\
\hline 14 & 31.736137 & 2084.511213 & 2022.130546 & 1901.094616 \\
\hline 15 & 2160.282968 & 2271.38594 & 2210.748616 & 2097.581584 \\
\hline 16 & 2390.670583 & 2470.446017 & 2349.046872 & 2207.099921 \\
\hline 17 & 2862.214873 & 102.18503 & 3204.397413 & 3132.506021 \\
\hline 18 & 3032.334758 & 183.224372 & 3240.198287 & 3155.133143 \\
\hline 19 & 3653.187355 & 4644.951507 & 4987.653933 & 4965.215177 \\
\hline 20 & 3891.907398 & 4802.664106 & 5055.47932 & 5007.433061 \\
\hline 21 & 4087.049651 & 5244.542959 & 5845.797103 & 5916.637283 \\
\hline 22 & 4310.870181 & 5609.431657 & 6072.135662 & 6109.213514 \\
\hline 23 & 4967.917359 & 5919.356572 & 6483.155425 & 6418.244519 \\
\hline 24 & 5071.33176 & 6372.640313 & 6811.332993 & 6679.5298 \\
\hline 25 & 5773.539558 & 6781.306359 & 7446.945858 & 7525.924043 \\
\hline 26 & 5832.68090 & & 7726.3391 & 7760.311456 \\
\hline 27 & 6782.990767 & 7855.5563 & 8626.395028 & 372.994018 \\
\hline 28 & 6819.313 & 8073.9 & 37 & 69.2139 \\
\hline 29 & & & & 3086 \\
\hline 30 & 1 & & & 987 \\
\hline 31 & 2.400 & & & 10592.24438 \\
\hline 32 & 395 & & & 10693.5242 \\
\hline 33 & & & & 11643.80223 \\
\hline 34 & 8534 & & 04 & 11671.35646 \\
\hline 35 & .63625 & 4 & 44 & 15009.53452 \\
\hline 36 & 10669.67764 & 3756403 & 14939.97457 & 15012.16227 \\
\hline 37 & 11260.96297 & 14359.76031 & 16166.92427 & 17851.6214 \\
\hline 38 & 11261.7744 & 4410.78132 & 16188.82351 & 17853.97145 \\
\hline 39 & 13198.53503 & 6700.91335 & 9154.70775 & 21239.4282 \\
\hline 40 & 13198.58502 & 16720.31099 & 19166.86589 & 21246.03279 \\
\hline 41 & 14397.21269 & 17906.69673 & 22138.63227 & 23102.63183 \\
\hline 42 & 14397.23309 & & 22171.82231 & 23116.20459 \\
\hline 43 & 15220.09027 & & 24061.96944 & 25736.81951 \\
\hline 44 & & & 24169750 & 25770.33133 \\
\hline 45 & & & & 278 \\
\hline 46 & 296 & & 8226 & 6.64662 \\
\hline 47 & 281 & & 26913.9 & 28601.6936 \\
\hline 48 & 5 & & 17 & 28754.16174 \\
\hline 49 & 9063 & 6 & 8477 & 30793.53445 \\
\hline 50 & 907 & 25700 . & 5876 & 30841.63357 \\
\hline 51 & 22783.03703 & & & 34134.31846 \\
\hline 52 & 22783.03703 & 26553.31359 & 31466.70217 & 34161.15367 \\
\hline 53 & 23433.84958 & 28213.6788 & 33262.0093 & 37167.81211 \\
\hline 54 & 23433.84958 & 28215.58649 & 33403.19865 & 37198.58281 \\
\hline 55 & 25351.5334 & 29443.75655 & 33931.28181 & 40833.49261 \\
\hline 56 & 25351.5334 & 29444.3795 & 34027.61129 & 40929.61047 \\
\hline 57 & 27715.38255 & 33742.34761 & 40150.23673 & 42690.50214 \\
\hline 58 & & 33742.47023 & 40197.5881 & 43007.41972 \\
\hline 59 & & & & \\
\hline 60 & & & & \\
\hline 61 & & & & 46847.27639 \\
\hline 62 & 30433.62543 & 38115.31519 & 43808.84607 & 46912.24644 \\
\hline 63 & 32301.2602 & 39168.36533 & 45119.54855 & 49225.86082 \\
\hline 64 & 32301.2602 & 39168.38327 & 45129.02125 & 49257.94419 \\
\hline
\end{tabular}



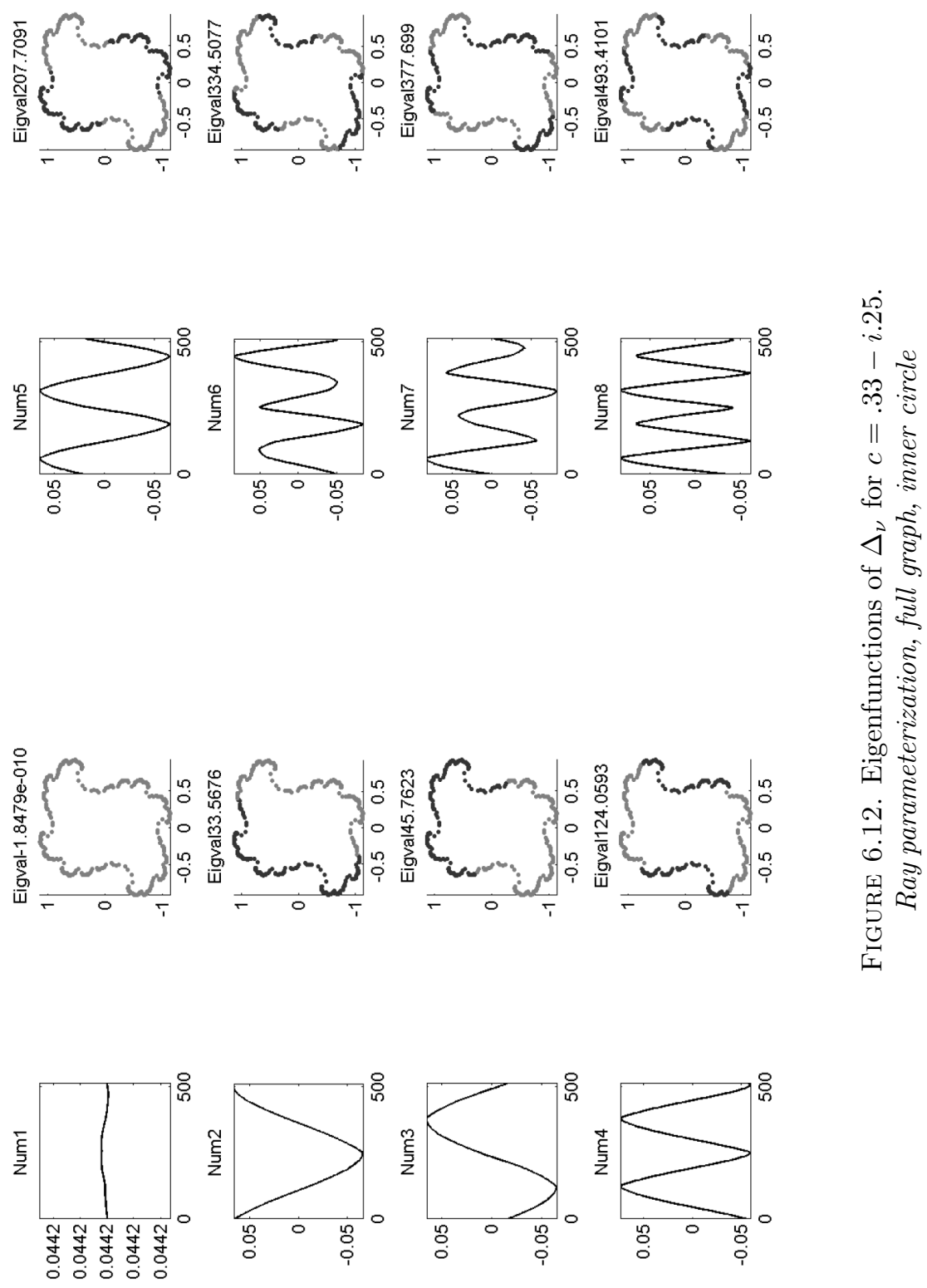
(ii.) Spectrum of $\Delta_{\nu}$ for $c=-1$

TABle 4. Computed actual eigenvalues of $\Delta_{\nu} c=-1$

\begin{tabular}{cccccc}
\hline$\#$ & Level 9 & Level 10 & Level 11 & Level 12 & Level 13 \\
\hline 1 & 0 & 0 & 0 & 0 & 0 \\
2 & 60.993 & 59.761 & 60.776 & 59.949 & 60.628867 \\
3 & 182.781 & 184.375 & 183.037 & 184.066 & 183.189141 \\
4 & 250.142 & 254.048 & 250.815 & 253.467 & 251.225964 \\
5 & 408.589 & 416.593 & 412.107 & 416.718 & 413.460382 \\
6 & 607.593 & 598.198 & 608.204 & 601.322 & 607.494978 \\
7 & 937.63 & 901.597 & 930.195 & 905.794 & 925.308868 \\
8 & 937.63 & 901.597 & 930.195 & 905.794 & 925.308868 \\
9 & 1022.231 & 1029.651 & 1024.59 & 1028.42 & 1025.052159 \\
10 & 1160.736 & 1161.321 & 1166.692 & 1164.51 & 1167.417114 \\
11 & 1347.226 & 1413.452 & 1361.854 & 1403.747 & 1368.444548 \\
12 & 1565.244 & 1581.749 & 1568.929 & 1578.585 & 1569.386446 \\
13 & 2071.629 & 2027.882 & 2086.665 & 2050.588 & 2085.253372 \\
14 & 2108.431 & 2059.044 & 2127.713 & 2086.337 & 2126.473126 \\
15 & 2698.402 & 2748.683 & 2759.452 & 2776.753 & 2775.995933 \\
16 & 2773.467 & 2822.446 & 2847.363 & 2860.13 & 2866.581565 \\
17 & 3958.772 & 4236.727 & 4115.627 & 4272.308 & 4163.937776 \\
18 & 4109.522 & 4236.727 & 4226.164 & 4281.382 & 4255.618846 \\
19 & 4496.357 & 4261.632 & 4453.202 & 4281.382 & 4418.306492 \\
20 & 4496.357 & 4286.489 & 4453.202 & 4305.384 & 4418.306492 \\
21 & 4804.91 & 4973.398 & 4832.476 & 4938.417 & 4844.527057 \\
22 & 4804.91 & 4973.398 & 4832.476 & 4938.417 & 4844.527057 \\
23 & 5043.498 & 5167.456 & 5164.223 & 5190.534 & 5182.9662 \\
24 & 5299.332 & 5320.95 & 5399.135 & 5357.508 & 5401.490376 \\
25 & 5580.047 & 5597.046 & 5600.197 & 5604.838 & 5599.106515 \\
26 & 5639.196 & 5748.875 & 5760.21 & 5807.467 & 5798.602469 \\
27 & 6059.609 & 6120.725 & 6332.735 & 6159.407 & 6295.939516 \\
28 & 6368.979 & 6120.725 & 6332.735 & 6159.407 & 6295.939516 \\
29 & 6368.979 & 6835.93 & 6445.912 & 6786.398 & 6521.592066 \\
30 & 6687.094 & 7258.7 & 7094.039 & 7280.802 & 7156.022336 \\
31 & 8032.96 & 8759.128 & 8643.513 & 8934.386 & 8767.016104 \\
32 & 8760.168 & 9213.677 & 9169.495 & 9352.056 & 9219.785067
\end{tabular}

Eigenvalue counting functions $N(x)$ and Weyl ratios $W(x)$ of $\Delta_{\nu}$ for $c=-1$ are illustrated in Figure 6.13. 


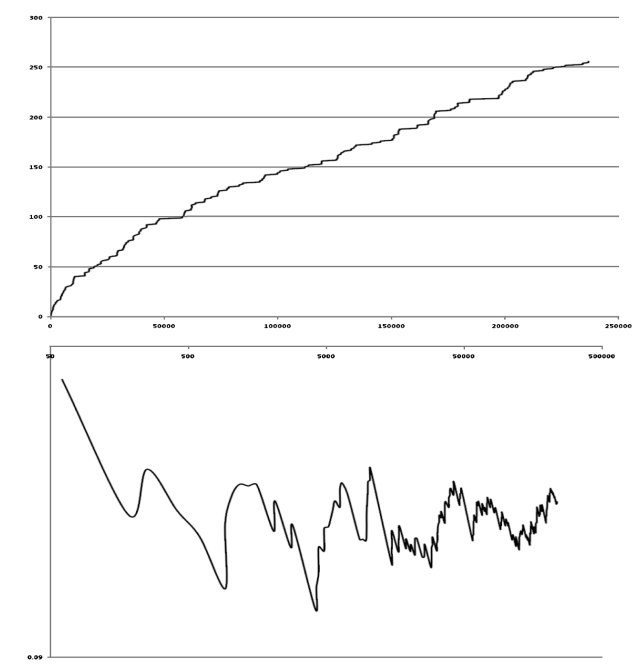

Figure 6.13. Top: Eigenvalue counting function. Bottom: Weyl ratio for level 13 eigenvalues of $\Delta_{\mu}$ for $c=-1$. 

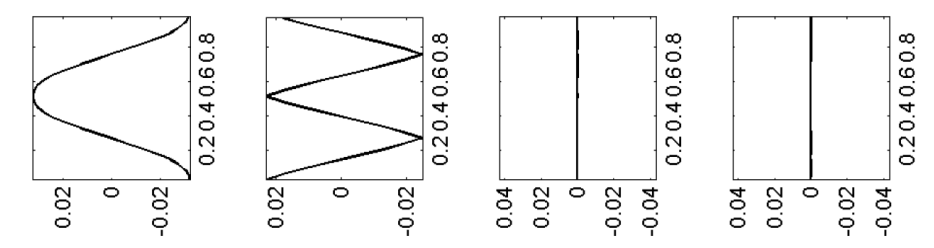

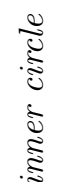
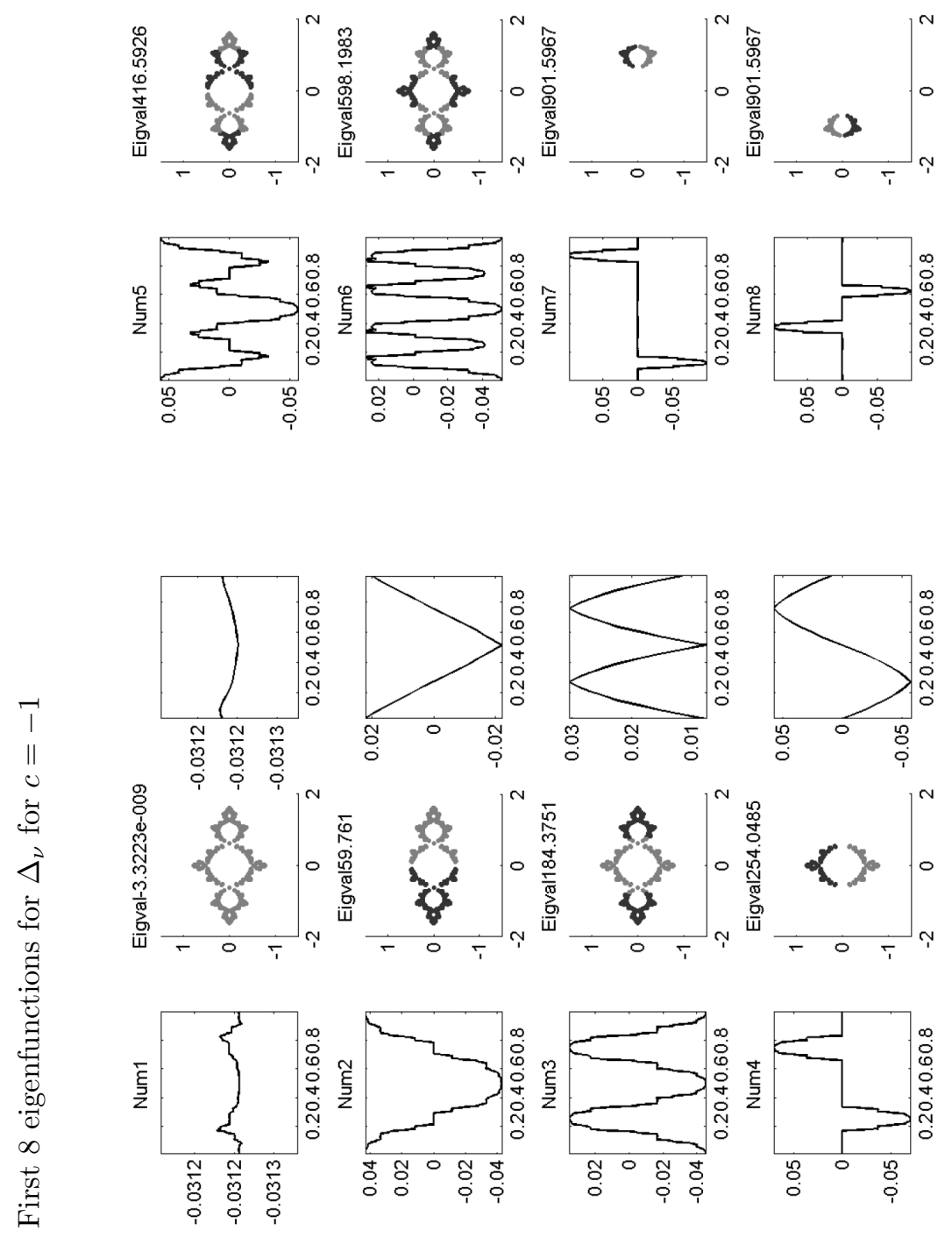

5
है
है

胥

हี

(1)

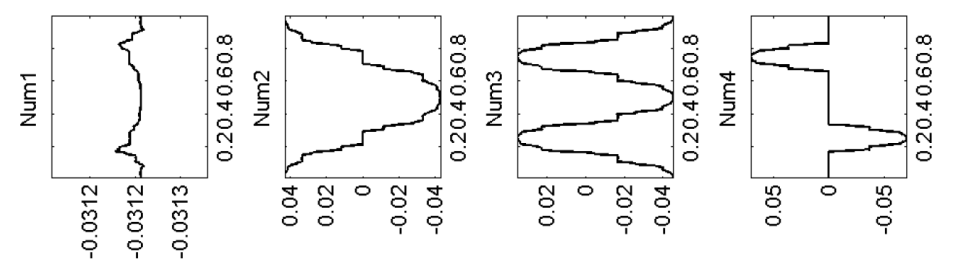


(iii.) Spectrum of $\Delta_{\nu}$ for $c=-.122+.745 i$

TABLE 5. Computed actual eigenvalues of $\Delta_{\nu} \mathrm{c}=-.122+754 \mathrm{i}$

\begin{tabular}{ccccc}
\hline$\#$ & Level 7 & Level 8 & Level 9 & Level 10 \\
\hline 1 & 0 & 0 & 0 & 0 \\
2 & 261.008937 & 256.664994 & 253.711999 & 259.52622 \\
3 & 813.033353 & 835.841454 & 818.641623 & 816.925874 \\
4 & 1050.51323 & 1090.320889 & 1050.878724 & 1063.443478 \\
5 & 1864.503404 & 1905.595532 & 1892.242852 & 1857.100836 \\
6 & 1932.097398 & 1996.110259 & 2032.413754 & 1954.662605 \\
7 & 2961.599964 & 2959.060298 & 3046.176418 & 3071.876939 \\
8 & 3556.548564 & 3414.215759 & 3472.053116 & 3546.823528 \\
9 & 4403.60581 & 4527.32098 & 4691.691621 & 4634.515807 \\
10 & 4717.04317 & 4850.516649 & 5024.370658 & 5072.002514 \\
11 & 6084.753065 & 5515.328777 & 5643.478349 & 5826.623251 \\
12 & 6138.707034 & 5540.670949 & 5671.383262 & 5881.593203 \\
13 & 6699.292031 & 7542.517574 & 7719.424833 & 7724.245096 \\
14 & 7090.00968 & 7732.659114 & 7854.390363 & 7965.143787 \\
15 & 7754.620735 & 11083.54177 & 12206.02323 & 11921.05164 \\
16 & 8059.503222 & 11769.58648 & 12345.74849 & 11992.92658
\end{tabular}

Because of the large differences in weight between points of a given level (as seen in Figure 6.10), our computational methods lose accuracy in this case. 

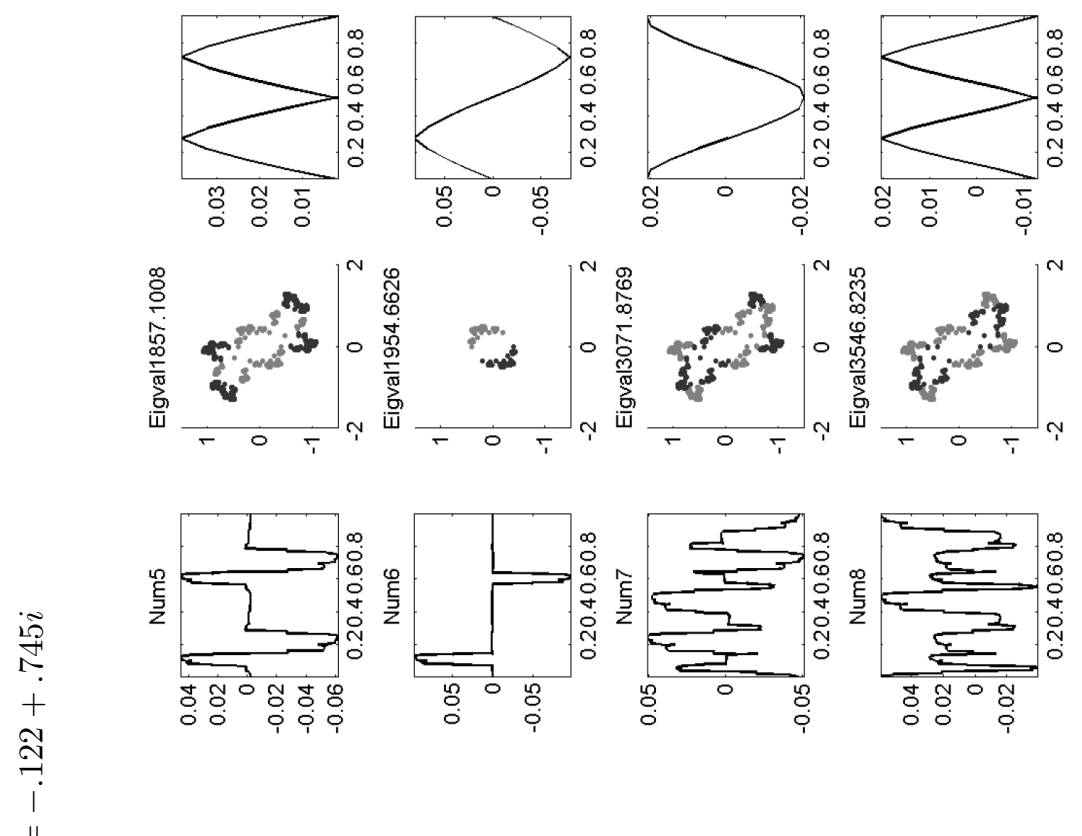

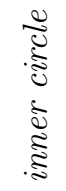

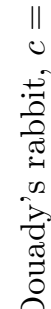
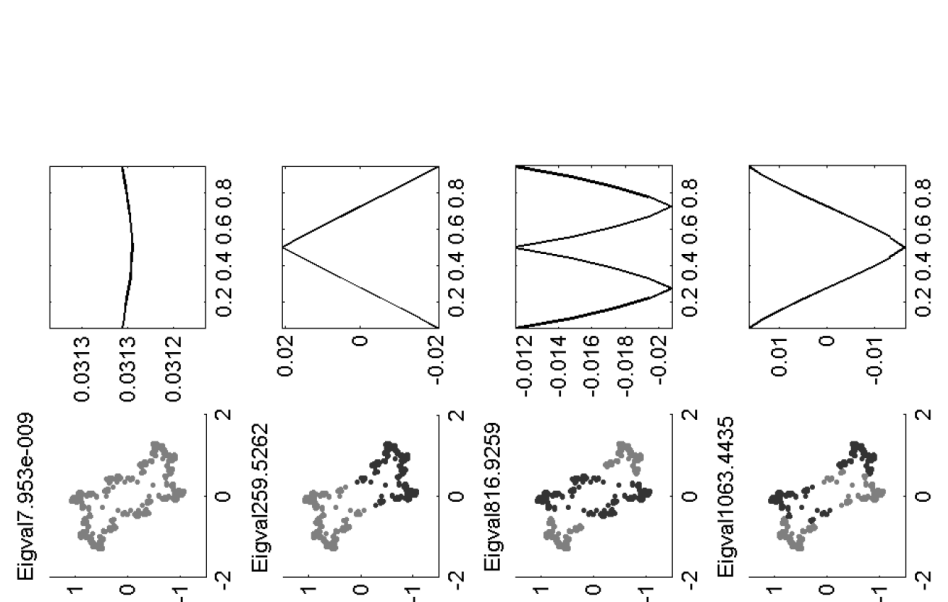

है

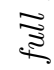

(ี)

है

ثี

4

$\stackrel{20}{0}$

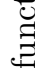

$\underset{8}{.0}$

蒡
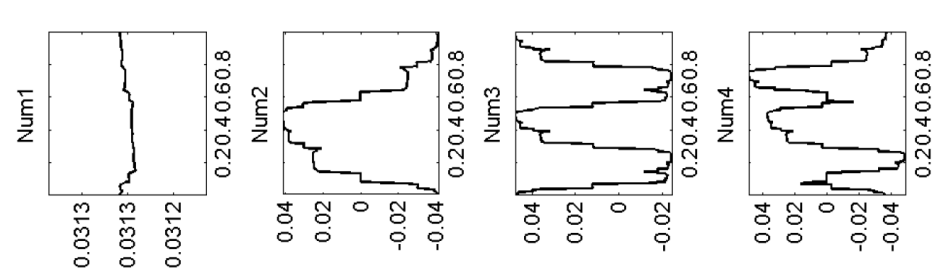


\section{Structure of the spectrum}

There is obviously a great deal of structure to the spectrum of the equilibrium Laplacian $\Delta_{\mu}$. As noted in [RT09] for the basilica, the eigenvalue counting function has the asymptotic behavior

$$
N(t) \sim t^{\frac{k}{k+1}} \text { as } t \rightarrow \infty
$$

and moreover the Weyl ratio $W(t)=\frac{N(t)}{t^{\frac{k}{k+1}}}$ approaches a multiplicatively periodic function

$$
W(t)=\varphi(t)+o(t) \text { as } t \rightarrow \infty
$$

with

$$
\varphi\left(2^{1+\frac{1}{k}} t\right)=\varphi(t)
$$

Here $\varphi$ is bounded and bounded away from 0. This follows from results of [KL93] in the general case by the same argument as in RT09. Informally, if we compare the eigenfunction $u(x)$ and $u(2 x)$, then we double the number of eigenvalues and multiply the eigenvalue by $2^{1+\frac{1}{k}}$ and by $2^{\left(1+\frac{1}{k}\right)\left(\frac{k}{k+1}\right)}=2$. We see this asymptotic periodic behavior in the graphs of $W(t)$ in Figures 6.8 and 6.10. In contrast, Figure 6.13 shows that this is not the case for the conformal Laplacian. The data suggests the following:

Conjecture 7.1. If $\lambda$ is an eigenvalue of $\Delta_{\mu}$, then

$$
N\left(2^{1+\frac{1}{k}} \lambda\right)=2 N(\lambda) \quad \text { or } \quad 2 N(\lambda)-1 .
$$

Note that $N(\lambda)$ is the number of the last eignfunction in the $\lambda$-eigenspace. For example, for the basilica $\lambda_{4}=196.54 \ldots, \lambda_{7}=\lambda_{8}=555.89 \approx 2^{\frac{3}{2}} \lambda_{4}, \lambda_{14}=\lambda_{15}=$ $\lambda_{16}=1572 \approx 2^{3} \lambda_{4}, \lambda_{27}, \ldots, \lambda_{32}=4445.29 \approx 2^{\frac{9}{2}} \lambda_{4}, \lambda_{54}, \ldots, \lambda_{64}=12554.04 \approx$ $2^{6} \lambda_{4}, \lambda_{107}, \ldots, \lambda_{128}=35445.04 \approx 2^{\frac{15}{2}} \lambda_{4}, \lambda_{214}, \ldots, \lambda_{256}=98999.38 \approx 2^{9} \lambda_{4}$. Also, $\lambda_{13}=1133.2, \ldots, \lambda_{25}=\lambda_{26}=2^{\frac{3}{2}} \lambda_{13}, \lambda_{49}=\lambda_{50}=\lambda_{51}=9.055 .3 \approx 2^{3} \lambda_{13}$, $\lambda_{97}, \ldots, \lambda_{102}=25566.53 \approx 2^{\frac{9}{2}} \lambda_{13} \lambda_{193}, \ldots, \lambda_{203}=71771.37 \approx 2^{6} \lambda_{13}$. In the first sequence we always have $N\left(2^{\frac{3}{2}} \lambda\right)=2 N(\lambda)$, while the second sequence alternates between that and $N\left(2^{\frac{3}{2}} \lambda\right)=2 N(\lambda)-1$.

We can speculate some more as to when the two alternatives in (7.4) occur. Suppose $\lambda$ is a primitive $H$ eigenvalue. Then the values $N\left(2^{\left(1+\frac{1}{k}\right) j} \lambda\right)=b_{j}$ will follow the pattern $b_{j+1}=2 b_{j}-\epsilon_{j}\left(\epsilon_{j}=0\right.$ or 1$)$ with $\epsilon_{j}$ periodic of period $k$. Moreover, $\left(\epsilon_{0}, \epsilon_{1}, \ldots, \epsilon_{k-1}\right)$ will depend on $\#(\lambda) \bmod 2^{k}$. Table 7.1 shows the correspondence for $k=2$ and 3 .

For $\lambda$ a primitive $V$ eigenfunction a similar statement holds, but the correspondence is different. We don't have a lot of data, but it appears that $b_{j}=2^{j} b_{0}$ when $\#(\lambda) \equiv 0 \bmod 2^{k}$. We note that eigenvalue \#53 for the basilica is a primitive eigenvalue of $H$ type, but its derived spaces have multiplicities equal to a $V$ type, and its $\epsilon$ sequence is $(0,1)$, which is the same as for $V$ types.

Assuming the above is correct, we may compute values for the Weyl ratio $W\left(2^{\left(1+\frac{1}{k}\right) j}\right) \lambda$ and $\varphi(\lambda)$ for these eigenvalues. For example, $\lambda_{2^{k}}$ is the first primitive $V$ eigenvalue, with $N\left(2^{\left(1+\frac{1}{k}\right) j} \lambda_{2^{k}}\right)=2^{j} 2^{k}$ and $W\left(2^{\left(1+\frac{1}{k}\right) j} \lambda_{2^{k}}\right)=\frac{2^{k}}{\lambda_{2^{k}}^{\left(\frac{k}{k+1}\right)}}=\varphi\left(\lambda_{2^{k}}\right)$. 
TABLE 7.1. $\left(\epsilon_{0}, \epsilon_{1}, \ldots, \epsilon_{k-1}\right)$ given $\#(\lambda) \bmod 2^{k}, k=2,3$.

\begin{tabular}{cccc}
$\#(\lambda) \bmod 4$ & $\left(\epsilon_{0}, \epsilon_{1}\right)$ & $\#(\lambda) \bmod 8$ & $\left(\epsilon_{0}, \epsilon_{1}, \epsilon_{2}\right)$ \\
\hline 0 & $(0,0)$ & 0 & $(0,0,0)$ \\
1 & $(1,1)$ & 1 & $(1,1,1)$ \\
2 & $(1,0)$ & 2 & $(1,0,0)$ \\
3 & $(0,1)$ & 3 & $(1,0,1)$ \\
& & 4 & $(1,0,0)$ \\
& & 5 & $(0,1,1)$ \\
& & 6 & $(0,1,0)$ \\
& & 7 (no data) & $(0,0,1)$
\end{tabular}

From the multiplicity formula in Theorem 7.3 we find

$$
\varphi^{-}\left(\lambda_{2^{k}}\right)=\lim _{\epsilon \rightarrow 0^{+}} \varphi\left(\lambda_{2^{k}}-\epsilon\right)=\frac{2^{k}-\left(\frac{2^{k}-2}{2^{k}-1}\right)}{\left(\lambda_{2^{k}}\right)^{\frac{k}{k+1}}} .
$$

In Figures 6.8 and 6.10 we see these values as local maxima and minima. The same will be true for other primitive $V$ eigenspaces, but quantitatively the effects may be much smaller. On the other hand, $\lambda_{2}$ is the first primitive $H$ eigenvalue, with $N\left(2^{\left(1+\frac{1}{k}\right) j} \lambda_{2}\right)=2 \cdot 2^{j}$ and $W\left(2^{\left(1+\frac{1}{k}\right) j} \lambda_{2}\right)={\frac{2}{\lambda_{2}}}^{\left(\frac{k}{k+1}\right)}=\varphi\left(\lambda_{2}\right)$, but this appears to be a local minimum, and similarly for the other primitive $H$ eigenvalues.

Conjecture 7.2. There are pairs of consecutive primitive eigenvalues $\left(H_{m}, V_{n}\right)$ or $\left(V_{n}, H_{m}\right)$ for every $n$ and the appropriate $m$, and those are the only pairs of consecutive primitive eigenvalues. Then $D_{j}\left(H_{m}\right), D_{j}\left(V_{n}\right)$ are consecutive eigenvalues for all $j$. In the case $n=1, \lambda * 2^{k+j}$ is $D_{j}\left(V_{1}\right)$.

We can explain the multiplicities as follows.

Theorem 7.3. Let $\lambda$ be a simple primitive eigenvalue associated to a $V$ eigenfunction, and let $2^{n\left(1+\frac{1}{k}\right)} \lambda$ be the $n$-th derived eigenvalue. Then the multiplicity mult $\left(2^{n\left(1+\frac{1}{k}\right)} \lambda\right)$ of the eigenspace is at least

$$
2^{j}\left(2^{m k}-\frac{2^{m k}-1}{2^{k}-1}\right), \quad n=m k+j, j=0,1, \ldots, k-1 .
$$

Proof. The $\lambda$-eigenfunction $u(x)$ is supported in the vertical segment and has symmetry type -+ . Then $u(2 x)$ is supported in the two vertical segments that are separated by the central vertical segment, so we can restrict it to either vertical segment and still have an eigenfunction. Thus the multiplicity is at least 2 when $n=1$. A similar argument shows that the number of vertical segments doubles as $n$ increases up to $k-1$, since each vertical segment gets mapped to two distinct vertical segments under $P^{-1}$. In terms of the parameterization, the central vertical segment is the union of two intervals, $\left[\frac{1}{2\left(2^{k}-1\right)}, \frac{2}{2\left(2^{k}-1\right)}\right] \cup\left[\frac{2^{k}}{2\left(2^{k}-1\right)}, \frac{2^{k}+1}{2\left(2^{k}-1\right)}\right]$. After $n$ iterations of $P^{-1}$ this is mapped into the union of $2^{m+1}$ intervals $\left[\frac{1+l(2 k-1)}{2^{n+1}\left(2^{k}-1\right)}, \frac{2+l(2 k-1)}{2^{n+1}\left(2^{k}-1\right)}\right]$ for $0 \leq l<2^{n+1}$. Note that these are exactly the shortest intervals in $\Gamma_{n+1}^{\prime}$. For $n<k$ they pair up to give $2^{n}$ vertical segments. However, when $n=k$ there are $2^{k}-2$ pairs of intervals that make up vertical segments, but the 4 intervals corresponding to $l=1,2,2^{k}, 2^{k}+1$ that lie in the central vertical segment form a single connected 
region (in this case the intersection of the central vertical segment and the central horizontal segment).

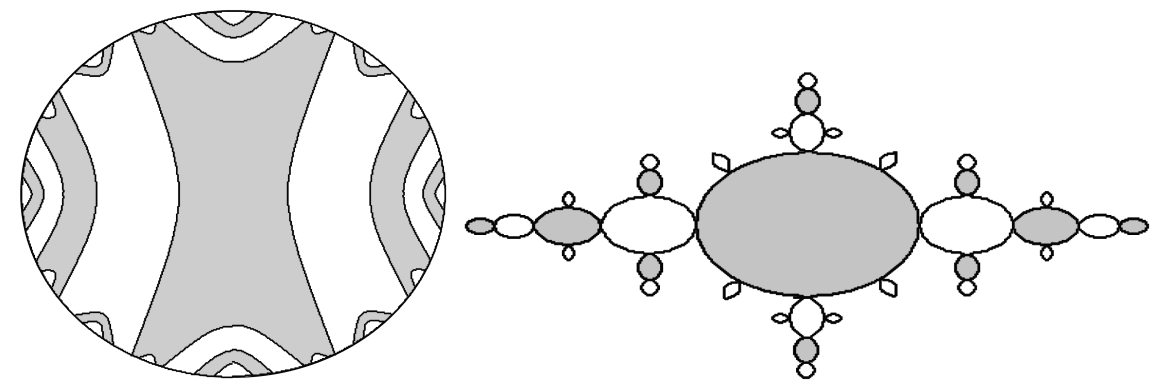

Figure 7.1. Left: The 11 connected components for $k=2, n=4$.

Right: The corresponding regions on the basilica.

Thus the lower bound for multiplicity when $n=k$ is $2^{k}-1$. The pattern then repeats, with

$$
\#(m k+j)=\left\{\begin{array}{cc}
2 \#(m k+j-1) & \text { if } j=1, \ldots, k-1 \\
2 \#(m k+j-1)-1 & \text { if } j=0
\end{array}\right.
$$

where \# $(n)$ denotes the number of connected components in the support of $u\left(P^{n} x\right)$. See Figures 7.1 and 7.2. Then \#(n) is given explicitly by (7.5).

There are few examples of eigenfunctions that display more complex behavior than outlined above, most notably 212-213 on the basilica. As the study of these examples is ongoing, more details may be found online [Flo08.

An immediate consequence of the high multiplicities in Theorem 7.3 is the following observation about the periodic function $\varphi$ in the Weyl ratio (7.2).

Corollary 7.4. The function $\varphi$ in (7.2) is discontinuous; hence in particular it is not constant.

Proof. Let $\lambda$ be a simple primitive eigenvalue of $V$ type. Then (7.5) implies $N\left(2^{n\left(1+\frac{1}{k}\right)} \lambda\right)-N\left(2^{n\left(1+\frac{1}{k}\right)} \lambda-\epsilon\right) \geq(7.5)$ for any $\epsilon>0$. For simplicity take $n=m k$. Then $W\left(2^{m(k+1)} \lambda\right)-W\left(2^{m(k+1)} \lambda-\epsilon\right) \geq \frac{2^{m k}-\frac{2^{m k}-1}{2^{k}-1}}{\left(2^{m(k+1)} \lambda\right)^{k+1}}>\frac{2^{m k}\left(\frac{2^{k}-2}{2^{k}}\right)}{2^{m k} \lambda^{k+1}}$, which implies that $\varphi$ has a jump discontinuity at $t=\lambda$ of at least $\left(2^{k}-2\right) 2^{-k} \lambda^{-\left(\frac{k}{k+1}\right)}$.

We may interpret the asymptotic behavior (7.1) as saying that $\mathcal{J}$ is a space of dimension $k$ in the resistance metric and $\Delta_{\mu}$ is an operator of order $k+1$. The resistance metric $R(x, y)$ is defined by

$$
R(x, y)^{-1}=\inf \{\mathcal{E}(u, u): u(x)=0 \quad \text { and } \quad u(y)=1\} .
$$

While it is tricky to compute $R(x, y)$ exactly, it is not difficult to obtain order of magnitude estimates. We claim that if $x$ and $y$ are adjacent vertices in $\Gamma_{m}$, then $R(x, y)$ is comparable to $2^{-\frac{m}{k}}$. To see this we first observe that we can replace $\mathcal{E}$ by $\mathcal{E}^{(j)}$ in (7.7) because of the estimate (3.17). The function that achieves the minimum in (7.7) is the harmonic extension of $u$ on $V_{m}$, so $\mathcal{E}^{(j)}(u, u)=\mathcal{E}_{m}(u, u) \geq 2^{\frac{m}{k}}$ (here $j \equiv m \bmod k$ ) because of the contribution from the edge joining $x$ and $y$. This gives 

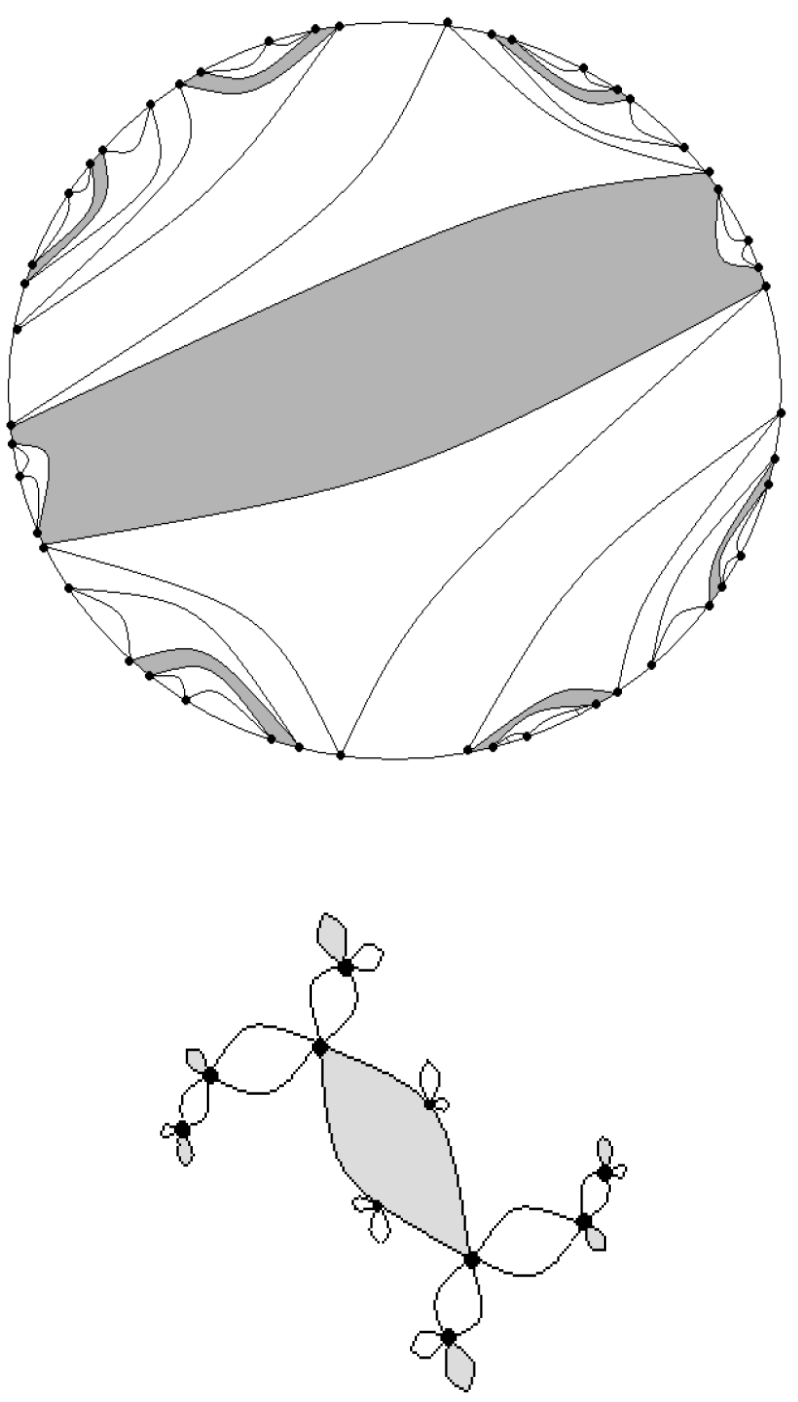

Figure 7.2. Top: The 7 connected components for $k=3, n=3$.

Bottom: The corresponding regions on the rabbit.

an upper bound for $R(x, y)$ of a multiple of $2^{-\frac{m}{k}}$, and we obtain a similar lower bound simply by filling in the values $u(z)=0$ for all the other vertices $z \in V_{m}$.

The measure $\mu$ of the subset of $\mathcal{J}$ corresponding to the intervals in the parameter space connecting $x$ and $y$ is on the order of $2^{-m}$. This translates into $\mu\left(B_{r}(x)\right) \sim r^{k}$, where $B_{r}(x)$ is the ball of radius $r$ in the resistance metric centered at $x$. This says the measure $\mu$ is $k$-dimensional. We may cover $\mathcal{J}$ with a multiple of $2^{m}$ such subsets, each with diameter on the order of $2^{-\frac{m}{k}}$, and this can be used to show that $\mathcal{J}$ has box dimension and Hausdorff dimension $k$ in the resistance metric. 
Theorem 7.5. Let $\lambda$ be a primitive eigenvalue with an eigenfunction $u$ of type $H$ (resp. V). Then u is supported on the central horizontal (resp. vertical) segment.

Proof. By symmetry we can restrict $u$ to any horizontal (resp. vertical) segment and still obtain a $\lambda$-eigenfunction. If $u$ is not supported in the central segment, then we can replace $u$ by $\tilde{u}$ supported in a disjoint pair of segments that are permuted under $R_{V}$ (resp. $\left.R_{H}\right)$. By symmetry we have $\tilde{u}\left(R_{V} x\right)=\tilde{u}(x)$ and $\tilde{u}\left(R_{H} x\right)=-\tilde{u}\left(R_{H} x\right)$ (resp. interchange $R_{V}$ and $R_{H}$ ). We can then define yet another $\lambda$-eigenfunction by multiplying $\tilde{u}$ by -1 on one of the two segments. This eigenfunction has symmetry type - - and hence is derived, and this contradicts the assumption that $\lambda$ is primitive.

Note that this theorem applies to primitive eigenfunctions of higer multiplicity. Since those spaces may have eigenfunctions that are linear combinations of $H$ and $V$ types, the theorem does not say anything about the supports of all primitive eigenfunctions. In section 8 we will show that the support of any eigenfunction cannot be all of $\mathcal{J}$.

Because of the rich structure of the eigenvalues and eigenfunction of $\Delta_{\mu}$, one is tempted to believe that some form of spectral decimation is present that would explain everything. While we cannot entirely rule this out, it is easy to see that spectral decimation does not hold for our sequence of graphs. Roughly speaking, spectral decimation would mean that eigenfunctions of the discrete Laplacians on $\Gamma_{m}$ would extend to eigenfunctions of $\Delta_{\mu}$ on $\mathcal{J}$, with eigenvalues changing in a predictable and monotonic fashion. However, the eigenfunctions on $\Gamma_{m}$ for small $m$ favor the +- and ++ types, since the points $x$ of $V_{m}^{\prime}$ are mostly identified with $R_{V} x$. For example, for the basilica, there are no odd functions under $R_{V}$ for $m=1,2$ and just 1 out of 8 for $m=3$. But the data shows that eigenfunction 4 already has a -+ symmetry type. The situation is perhaps similar to the case of the pentagasket ASST03. We note that RT09] describes a Laplacian on the basilica that does enjoy spectral decimation, but it is built from a different energy.

Two other features of the spectrum of the Laplacians (these are shared with $\left.\Delta_{\nu}\right)$ are apparent from the data: spectral gaps and spectral clusters. Spectral gaps mean there is a sequence $\left\{n_{j}\right\}$ with

$$
\frac{\lambda_{n_{j}+1}-\lambda_{n_{j}}}{\lambda_{n_{j}}} \geq r>1
$$

In other words, not only is the gap between successive eigenvalues large, but it is large relative to the eigenvalues (this rules out the Laplacian on the sphere, for example). In fractals with spectral decimation, such as the Sierpinski gasket and the Vicsek set, it is possible to prove the existence of spectral gaps (FS92, Str06, [Zho09, Zho10]). For the pentagasket, as here, there is only experimental evidence ASST03. Spectral gaps seem to occur right after derived vertical eigenspaces.

Spectral clustering is the phenomenon of distinct eigenvalues being very close. In its extreme form, which we believe holds in our case, it would say

$$
\forall n \forall \epsilon>0 \exists \text { an interval I of length } \epsilon \text { containing } n \text { distinct eigenvalues. }
$$

For example, in the basilica between eigenfunction 185-189 there are 3 distinct eigenvalues in the interval [68978.39921,68978.42095] of length .02174. One is tempted to suppose that this might be a single eigenspace with the differences in eigenvalues due to a combination of round-off errors and the inaccuracy due to 
the approximation process. However, the data remains consistent over several levels of approximation, so we are inclined to believe the eigenvalues are indeed distinct. In the case of the Vicsek set, spectral clustering will be proved in [CSW09. It does not hold for the Sierpinski gasket. Experimental evidence in ASST03 suggests that it holds for the pentagasket.

In contrast to the equilibrium Laplacian, the conformal Laplacians $\Delta_{\nu}$ do not exhibit the same amount of spectral structure. Nevertheless, there are some interesting features that we should mention. First we discuss two examples of quasicircles, whose eigenfunctions are displayed in Figure $6.12(c=.33-i .25)$ and online $(c=.24)$ Flo08, In the case of the ordinary circle $(c=0)$, after the trivial 0 -eigenspace of constants the eigenspaces have multiplicity 2 with eigenfunctions $\cos 2 \pi k x$ and $\sin 2 \pi k x$ with eigenvalue $(2 \pi k)^{2}$. In both examples the eigenfunctions (in external ray parameterization) look like perturbations of the sines and cosines, at least for small values of $k$. But the multiplicity 2 eigenspaces are replaced by pairs of multiplicity 1 eigenspaces. In the second example, the horizontal and vertical reflections in the parameter space preserve $|z|$ on $\mathcal{J}$, hence they are symmetries of the measure $\nu$ and thus of $\Delta_{\nu}$ as well. So each eigenfunction is either symmetric or skew-symmetric under each symmetry. When $k$ is odd the eigenspaces are symmetric/skew-symmetric and skew-symmetric/symmetric, while when $k$ is even the eigenspaces are symmetric/symmetric and skew-symmetric/skew-symmetric. There does not seem to be a pattern to predict which of the two has the lower eigenvalue. In the first example there are no longer horizontal and vertical symmetries, only $z \rightarrow-z\left(t \rightarrow t+\frac{1}{2}\right.$ in the ray parameterization). Nevertheless the first few eigenfunctions closely resemble the sines and cosines.

The eigenfunctions of the conformal Laplacian on the basilica and rabbit are shown in Figures 6.14 and 6.15 and resemble the eigenfunctions of the equilibrium Laplacian in Figures 6.9 and 6.11, at least near the bottom of the spectrum. The conformal measures are preserved by the horizontal and vertical reflections, but there are no longer derived eigenspaces, since the action of $P$ distorts these measures. In both cases eigenfunction \#2 is of $H$ type with reduced support, but eigenfunction \#3 only approximates a doubling of \#2 and has full support. It is symmetric under both reflections. Basilica \#4 is of $V$ type, while \#11 is of both $H$ and $V$ types (it is skew-symmetric under both reflections). We also see eigenspaces of multiplicity two, for example \#7 and \#8, or \#21 and \#22, corresponding to pairs of regions illustrated in Figure 7.1 for which $|z|$ is identical. We never see multiplicity higher than two because among such corresponding regions there are never more than two with the same $|z|$ values. On the rabbit we see $H$ types (\#2) and $V$ types (\#6), but unfortunately we have not been able to get reliable accuracy higher up in the spectrum to see the other types of behavior, but these undoubtedly occur. Already \#15 and \#16 show puzzling behavior, as they are neither symmetric nor skew-symmetric with respect to the $z \rightarrow-z$ symmetry, yet they have different eigenvalues so they do not form a multiplicity 2 eigenspace. It would take higher resolution than we can attain to resolve the ambiguity, and for this reason we do not attempt to go higher up in the spectrum.

Finally we consider eigenfunctions for the conformal Laplacian on a distorted basilica (pictures can be found in [Flo08]). This example does not have horizontal and vertical symmetries (as also is the case for the quasicircle in Figure 6.12). The first few eigenfunctions appear to be perturbations of the ones in Figure 6.14. We 
note that some, like \#2, appear to have the same restricted support as $H$ type eigenfunctions. We do not know how to explain this or why there do not appear to be any with the restricted support of $V$ type eigenfunctions. It appears that all eigenspaces have multiplicity 1.

Another striking observation that we are not presently able to explain is the zigzag behavior of the restriction to the central circle of the eigenfunctions of the equilibrium Laplacian. This behavior is more striking for the rabbit than for the basilica, which leads us to speculate that there might be a general statement for all eigenfunctions in the limit as $k \rightarrow \infty$. (We also see the same behavior for \#2 for the conformal Laplacian.) More generally, it would be interesting to understand the behavior of eigenfunctions of a fixed \# and $k \rightarrow \infty$. In the case of the Viscek sets discussed in [CSW09, it is possible to answer such questions. Unfortunately, our numerical methods are not adequate to understand even the case $k=4$.

\section{Circles AND LOOPS}

We single out two types of subsets of $\mathcal{J}$ that we call circles and loops. Suppose $x$ is a point in $V_{m} \backslash V_{m-1}$. Then there are $k$ points $x_{1}, x_{2}, \ldots, x_{k}$ in $V_{m}^{\prime}$ in increasing order that are identified to obtain $x$. The portion of $\mathcal{J}$ parameterized by the interval $\left[x_{j}, x_{j+1}\right](j=1, \ldots, k-1)$ is called a loop. Note that there are $k-1$ loops attached at the point of $\mathcal{J}$ parameterized by $x$. The length of the loops is defined to be $x_{j+1}-x_{j}=\frac{2^{j-m}}{2^{k}-1}$.

The central circle is parameterized by the complement (essentally a Cantor set) of the interior of all loops. Note that $V_{1}^{\prime}$ has $2 k$ points that divide $C$ into $2 k$ intervals. Of these, $2 k-2$ correspond to loops. After we remove these, we are left with the two intervals $\left[\frac{1}{2\left(2^{k}-1\right)}, \frac{1}{2^{k}-1}\right]$ and $\left[\frac{1}{2}+\frac{1}{2\left(2^{k}-1\right)}, \frac{1}{2}+\frac{1}{2^{k}-1}\right]$. The central circle has parameters lying in the union of those intervals. (Note that if $k>2$ there will be isolated points outside those intervals that are identified with the endpoints, but we do not need to include these in the parametrization.) More generally, a circle is parametrized by a Cantor set obtained by all remaining interiors of loops from a pair of intervals $[x, y],\left[y^{\prime}, x^{\prime}\right]$, where $x, y$ and $y^{\prime}, x^{\prime}$ are consecutive but not identified points in $V_{m}$ and $x$ is identified with $x^{\prime}$ and $y$ is identified with $y^{\prime}$. We define the length of the circle to be $2(y-x)=\frac{2}{2^{m}\left(2^{k}-1\right)}$. It is not hard to see that the circles on $\mathcal{J}$ are indeed topological circles, and any two are either disjoint or intersect at a single point. There are a countable number circles and their union is dense in $\mathcal{J}$, but the union is not all of $\mathcal{J}$, as it has $\mu$-measure zero.

Suppose $u$ is a function defined on the central circle. It is natural to extend $u$ to $\tilde{u}$ on $\mathcal{J}$ by making it constant on all loops, as this clearly minimizes energy. We can easily compute the energy $\mathcal{E}_{n}(\tilde{u}, \tilde{u})$. It is natural to separate it into two terms corresponding to the initial intervals $I=\left[\frac{1}{2\left(2^{k}-1\right)}, \frac{1}{2^{k}-1}\right]$ and $I^{\prime}=I+\frac{1}{2}$. Note that $I$ does not subdivide in $V_{2}^{\prime}, \ldots, V_{k}^{\prime}$, and in $V_{k+1}^{\prime}$ it splits into the two outer intervals of length $\frac{1}{2^{k}}|I|$ and $k-1$ loops that do not contribute to the energy. This process then repeats. Thus the parameter space of the $I$ half of the central circle is exactly a linear Cantor set with dissection ratio $\frac{1}{2^{k}}$. In $V_{m k+1}^{\prime}$ we have $2^{m}$ intervals of length $\frac{1}{2^{m k}}|I|$, with consecutive endpoints identified in $V_{m k+1}$. This leads to a new parameterization of half of the central circle $\varphi(s)$ with $s \in[0,1]$ as follows: map the values $\left[\frac{l}{2^{m}}\right]$ to the points in $\mathcal{J}$ parameterized in order by the points in $V_{m k+1}$ at the endpoints of the intervals. At each step, passing from $m$ to $m+1$, we insert 
one new point in $V_{(m+1) k+1}$ in between each consecutive pair of points in $V_{m k+1}$ from the previous step, so the definition is consistent going from $m$ to $m+1$. This defines $\varphi(s)$ on all dyadic rationals, and we extend it by continuity to $[0,1]$. A similar parameterization works for the other half on $[-1,0]$, and the two join up at the endpoints to give a parametrization of the central circle by a standard circle of circumference 2 .

Now we compute for the central circle

$$
E_{m k+1}(\tilde{u}, \tilde{u})=2^{m k+1}\left(2^{k}-1\right) 2^{m} \sum_{l=-2^{m}}^{2^{m}-1}\left|u\left(\varphi\left(\frac{l+1}{2^{m}}\right)\right)-u\left(\varphi\left(\frac{l}{2^{m}}\right)\right)\right|^{2},
$$

SO

$$
\begin{array}{r}
\mathcal{E}_{m k+1}(\tilde{u}), \tilde{u}=2^{\frac{1}{k}}\left(2^{k}-1\right) \sum_{l=-2^{m}}^{2^{m}}\left|u\left(\varphi\left(\frac{l+1}{2^{m}}\right)\right)-u\left(\varphi\left(\frac{l}{2^{m}}\right)\right)\right|^{2} \\
=2^{\frac{1}{k}}\left(2^{k}-1\right) \tilde{\mathcal{E}}(u \circ \varphi, u \circ \varphi),
\end{array}
$$

where $\tilde{\mathcal{E}}_{m}$ is the standard dyadic energy on [0,1]. Taking the limit as $m \rightarrow \infty$ yields

$$
\mathcal{E}^{(1)}(\tilde{u}, \tilde{u})=2^{\frac{1}{k}}\left(2^{k}-1\right) \tilde{\mathcal{E}}(u \circ \varphi, u \circ \varphi),
$$

where $\tilde{\mathcal{E}}$ is the standard energy ( $H^{1}$ Sobolev space) on the parameter space. Note that $E_{m k+j}(\tilde{u}, \tilde{u})=E_{m k+1}(\tilde{u}, \tilde{u})$ for $1 \leq j \leq k$ because no new points are added to the parametrization of the central circle in the passage from $V_{m k+l}$ to $V_{m k+l+1}$ for $1 \leq l \leq k-1$. By $(3.11)$

$$
\mathcal{E}_{m k+j}(\tilde{u}, \tilde{u})=2^{\left(\frac{1-k}{k}\right)(j-1)} \mathcal{E}_{m k+1}(\tilde{u}, \tilde{u}),
$$

SO

$$
\mathcal{E}^{(j)}(\tilde{u}, \tilde{u})=2^{1-j\left(\frac{k-1}{k}\right)}\left(2^{k}-1\right) \mathcal{E}(u \circ \varphi, u \circ \varphi)
$$

(here we use $j=k$ rather than $j=0$ for $\left.\mathcal{E}^{(0)}\right)$. Thus

$$
\mathcal{E}(\tilde{u}, \tilde{u})=c_{k} \mathcal{E}(u \circ \varphi, u \circ \varphi)
$$

for

$$
c_{k}=\frac{2\left(2^{k}-1\right)\left(1-2^{1-k}\right)}{k\left(2^{1-\frac{1}{k}}-1\right)} .
$$

More generally, suppose we consider a circle $C_{n}$ of length $\frac{2}{2^{m}\left(2^{k}-1\right)}$ that arises in $V_{m}$ (the central circle has $m=1$ ). Again we may parameterize it by $[-1,1]$ and write $\varphi_{n}$ for the parametrization map. If $u$ is defined on the circle and $\tilde{u}$ is its harmonic extension, then

$$
\mathcal{E}(\tilde{u}, \tilde{u})=2^{\frac{m-1}{k}} c_{k} \mathcal{E}\left(u \circ \varphi_{n}, u \circ \varphi_{n}\right) .
$$

Let $C_{0}, C_{1}, C_{2}, \ldots$ be the list of all circles in $\mathcal{J}$, with $C_{0}$ the central circle, and let $m(n)$ be the value of $m$ assoicated with $C_{n}$. The following result was established for $k=2$ in [RT09]:

Theorem 8.1. Let $u$ be a continuous function on $\mathcal{J}$. Then $u \in d o m \mathcal{E}$ if and only if $u \circ \varphi_{n} \in \operatorname{dom} \tilde{\mathcal{E}}$ for every $n$ and

$$
\mathcal{E}(u, u)=\sum_{n=0}^{\infty} 2^{\frac{m(n)-1}{k}} c_{k} \tilde{\mathcal{E}}\left(u \circ \varphi_{n}, u \circ \varphi_{n}\right)
$$

is finite. 
Proof. Suppose $u \in \operatorname{dom} \mathcal{E}$. Let $\tilde{u}_{N}$ denote the harmonic extension of the restriction of $u$ to $C_{0} \cup C_{1} \cup \ldots \cup C_{N}$. Then by the previous argument

$$
\mathcal{E}\left(\tilde{u}_{N}, \tilde{u}_{N}\right)=\sum_{n=0}^{N} 2^{\frac{m(n)-1}{k}} c_{k} \tilde{\mathcal{E}}\left(u \circ \varphi_{n}, u \circ \varphi_{n}\right) .
$$

It is routine to show $\mathcal{E}(u, u)=\lim _{N \rightarrow \infty} \mathcal{E}\left(\tilde{u}_{N}, \tilde{u}_{N}\right)$, so (8.9) holds; in particular, the sum is finite and $u \circ \varphi_{n} \in \operatorname{dom} \tilde{\mathcal{E}}$ for each $n$.

Conversely, suppose $u$ is continuous and the sum in (8.9) is finite. Then if we define $\tilde{u}_{N}$ as above, the previous argument shows that $\tilde{u}_{N} \in$ dom $\mathcal{E}$ and (8.10) holds. Then $\tilde{u}_{N}$ is a Cauchy sequence in energy and so converges to an element in $\operatorname{dom} \mathcal{E}$ that we may identify with $u$.

There is a close relationship between the set of circles and the supports of the derived vertical eigenspaces as described in the proof of Theorem 7.3. Each of the regions contains a circle and some loops attached to it (see Figures 7.2 and 7.4). Each circle occurs infinitely often, with fewer attached loops as the order of the derived eigenvalue increases.

We note that $P^{(k)}$ maps $C_{0}$ to $C_{0}$ in a two-to-one fashion and is conjugate $\left(\varphi_{0}^{-1} P^{(k)} \varphi_{0}\right)$ to the doubling map on the parameter circle. Since the doubling map multiplies the standard energy $\tilde{\mathcal{E}}$ by 4 , it follows that the contribution from $C_{0}$ to the total energy is also multiplied by 4 :

$$
c_{k} \tilde{\mathcal{E}}\left(u \circ P^{(k)} \circ \varphi_{0}, u \circ P^{(k)} \circ \varphi_{0}\right)=4 c_{k} \tilde{\mathcal{E}}\left(u \circ \varphi_{0}, u \circ \varphi_{0}\right) .
$$

One can also derive (8.11) directly from the definition. Although (8.11) appears to conflict with (3.26), it has to be noted that the inverse image of $C_{0}$ under $P^{(k)}$ is much larger than $C_{0}$, and the entire inverse image contributes to the left side of (3.24). In passing from the global energy identity to the local one the value is reduced by a factor of $\frac{1}{2^{k-1}}$, so the factor $2^{k+1}$ in (3.24) becomes 4 in (8.11). There are similar but more complicated identities relating to the transformation of energies on other circles under the iteration of $P$.

It is useful to decompose the sum in (8.9) into the contributions from circles with $m(n)$ in a given residue class modulo $k$. So we define

$$
\tilde{\mathcal{E}}^{(j)}(u, u)=\sum_{m(n) \equiv j(k)} 2^{\frac{m(n)}{k}}\left(2^{k}-1\right) \tilde{\mathcal{E}}\left(u \circ \varphi_{n}, u \circ \varphi_{n}\right) \text { for } j=0,1, \ldots, k-1 .
$$

Now suppose we fix a circle $C_{n}$ with $m(n) \equiv j(k)$ and let $\tilde{u_{n}}$ be the harmonic extension of $\left.u\right|_{C_{n}}$. The analogs of $(8.1),(8,2)$, and (8.3) are

$$
\begin{aligned}
& E_{m(n)+m k}\left(\tilde{u_{n}}, \tilde{u_{n}}\right)=2^{m(n)+m k}\left(2^{k}-1\right) \sum_{l=-2^{m}}^{2^{m}-1}\left|u\left(\varphi_{n}\left(\frac{l+1}{2^{m}}\right)\right)-u\left(\varphi_{n}\left(\frac{l}{2^{m}}\right)\right)\right|^{2}, \\
& \mathcal{E}_{m(n)+m k}\left(\tilde{u_{n}}, \tilde{u_{n}}\right)=2^{\frac{m(n)}{k}}\left(2^{k}-1\right) \tilde{\mathcal{E}}_{m}\left(u \circ \varphi_{n}, u \circ \varphi_{n}\right)
\end{aligned}
$$

and

$$
\mathcal{E}^{(j)}\left(\tilde{u_{n}}, \tilde{u_{n}}\right)=2^{\frac{m(n)}{k}}\left(2^{k}-1\right) \tilde{\mathcal{E}}_{m}\left(u \circ \varphi_{n}, u \circ \varphi_{n}\right) .
$$

There will be no new subdivision points in $C_{n}$ when we pass from $m(n)+m k$ to $m(n)+m k+p$ for $p \leq k-1$, so

$$
E_{m(n)+m k+p}\left(\tilde{u_{n}}, \tilde{u_{n}}\right)=E_{m(n)+m k+p}(\tilde{u}, \tilde{u})
$$


SO

$$
\mathcal{E}^{(j+p)}\left(\tilde{u}_{n}, \tilde{u}_{n}\right)=2^{\frac{m(n)+(1-k) p}{k}}\left(2^{k}-1\right) \tilde{\mathcal{E}}\left(u \circ \varphi_{n}, u \circ \varphi_{n}\right) \text { for } 0 \leq p \leq k-1 .
$$

If we sum over all circles and sort according to residue classes of $m(n)$, we obtain, for $0 \leq q \leq k-1$,

$$
\mathcal{E}^{(q)}(u, u)=\sum_{j=0}^{k-1} 2^{\left(\frac{1-k}{k}\right)[q-j]} \tilde{\mathcal{E}}^{(j)}(u, u),
$$

where we interpret $[q-j]$ as $q-j$ if $j \leq q$ and $k+q-j$ if $j>q$. Note that for each fixed $j$ the values of $[q-j]$ cycle through the integers $0,1, \ldots, k-1$. By averaging (8.18) over $q$ we recover (8.9).

We also note that, although each circle has $\mu$-measure zero, we can express the integral of continuous function in terms of the ordinary integrals of its restrictions to all the circles as a limit of sums. Suppose we fix a circle $C_{n}$ with $m(n)=m$. The length of the circle is $\frac{2}{2^{m}\left(2^{k}-1\right)}$, and there are two intervals of half this length in $C \backslash V_{m}$ that parameterize $C_{n}$ and all the loops to which they attach. We may approximate the integral over these intervals by

$$
\frac{1}{2^{m}\left(2^{k}-1\right)} \int_{-1}^{1} f\left(\varphi_{n}(t)\right) d t
$$

The same approximation is valid for $C \backslash V_{m+j}$ for $j \leq k$, but in $C \backslash V_{m+k}$ the intervals subdivide and only two intervals of length $\frac{2}{2^{m+k}\left(2^{k}-1\right)}$ parameterize $C_{n}$ and some of the loops that attach to it. In general, in $C \backslash V_{j}$ with $j \geq m$, the intervals representing $C_{n}$ will be subdivided $l=\left[\frac{j-m}{k}\right]$ times, and each time the total length is reduced by a factor of $\frac{1}{2^{k-1}}$. Thus

$$
\sum_{m(n) \leq j} \frac{1}{2^{m(n)+\left[\frac{j-m(n)}{k}\right](k-1)}\left(2^{k}-1\right)} \int_{-1}^{1} f\left(\varphi_{n}(t)\right) d t
$$

is the approximation at level $j$. It is easy to see that (8.20) converges to $\int_{\mathcal{J}} f d \mu$ as $j \rightarrow \infty$.

\section{Eigenfunctions on LOOPS AND CIRCles}

Consider a loop $\mathcal{L}$ of length $\frac{2^{j-m}}{2^{k}-1}$. We regard the point $z_{0}$ where the loops join the rest of $\mathcal{J}$ as the boundary point. In the parameter space $z_{0}$ corresponds to the two boundary points of the interval $I(\mathcal{L})$ that parameterizes $\mathcal{L}$. Everything else is interior. It makes sense to consider the eigenvalue equation on $\mathcal{L}$ :

$$
\begin{gathered}
-\Delta_{\mu} u=\lambda u \text { on } \mathcal{L} \backslash z_{0}, \\
u\left(z_{0}\right)=a .
\end{gathered}
$$

If there exists a nonzero solution with $a=0$, we say that $\lambda$ is a Dirichlet eigenvalue on $\mathcal{L}$. The set of Dirichlet eigenvalues forms a sequence

$$
0<\lambda_{1}(\mathcal{L}) \leq \lambda_{2}(\mathcal{L}) \leq \ldots \rightarrow \infty .
$$

Let $\lambda_{n}=\lambda_{n}(\mathcal{L})$, where $\mathcal{L}$ is a loop of the largest length $(m=1, j=1) \frac{1}{2^{k}-1}$. Then for a general loop we have from (4.8) that

$$
\lambda_{n}(\mathcal{L})=2^{\left(1+\frac{1}{k}\right)(m-j)} \lambda_{n},
$$


and the associated eigenfunctions are mapped to each other by appropriate iterations of $P$. We may regard these as similarities (they are similarities on the parameter space). Note that for any positive fixed $\lambda, \lambda_{1}(\mathcal{L})>\lambda$ for all sufficiently small loops (equivalently, all but a finite number of loops), and this implies that $\lambda$ is not a Dirichlet eigenvalue for such loops.

It is easy to see that (9.1) has a unique solution for any $\lambda$ that is not a Dirichlet eigenvalue. In particular, if $a=0$, then $u \equiv 0$. Let $u_{\lambda}$ denote the unique solution for $a=1$ when $\mathcal{L}$ is a loop of largest length $\frac{1}{2^{k}-1}$. Then the solution to the eigenvalue equation (9.1) on a general loop must have the form

$$
u\left(z_{0}\right) u_{2^{\left(1+\frac{1}{k}\right)(j-m)} \lambda} \circ S_{\mathcal{L}}
$$

provided $\lambda \neq 2^{\left(1+\frac{1}{k}\right)(j-m)} \lambda_{n}$ for any $n$, where $S_{\mathcal{L}}$ denotes the similarity mapping of the small loop to the large loop.

Now suppose $u$ is a global $\lambda$-eigenfunction on $\mathcal{J}$. Then for any sufficiently small loop, $u$ has the form (9.5).

Theorem 9.1. Let $u$ be a global eigenfunction of $\Delta_{\mu}$ on $\mathcal{J}$, and suppose $u$ is even or odd with respect to $R_{H}$ and $R_{V}$. Then there are loops on which u vanishes identically.

Proof. It suffices to prove this for primitive eigenfunctions, in which case $u$ has either +- or -+ symmetry. Hence it is odd with respect to either $R_{H}$ or $R_{V}$. Thus $u$ vanishes on the fixed points of $R_{H}$ or $R_{V}$, and there are infinitely many of these. If we choose a sufficiently small loop having one of these fixed points as boundary point $z_{0}$, then $u\left(z_{0}\right)=0$ and (9.5) shows that $u$ vanishes on the loop.

Conjecture 9.2. The same conclusion holds for all eigenfunctions.

The only way this can fail is for an eigenspace of nontrival multiplicity when $u$ is a linear combination of $H$ and $V$ primitive eigenfunctions. This happens on the basilica for a primitive eigenvalue in numbers 212 and 213 , yet the two eigenfunctions have loops where they both vanish. It also happens all the time for derived eigenvalues, but in all the examples the primitive eigenfunctions are locally equivalent to derived eigenfunctions.

Next we consider the restrictions of global eigenfunctions to circles. For simplicity we just consider the central circle $C_{0}$. In order to proceed we need to make the following assumptions on the eigenvalue $\lambda$ :

$$
\lambda \neq 2^{\left(1+\frac{1}{k}\right)(m-j)} \lambda_{n}
$$

for any $n$ and any $m$ and $j \leq k-1$. This makes (9.5) valid on any loop. Since $\mathcal{J}$ is the union of $C_{0}$ and all loops that attach to it, it follows that any $\lambda$-eigenfunction is uniquely determined by its restriction to $C_{0}$. (Of course there are eigenfunctions which vanish on $C_{0}$, so (9.6) does not always hold.) It should be possible to express the eigenvalue equation entirely in terms of the values of $u$ on points in $C_{0}$. We will indicate, at least in principle, how this can be done.

The points in $V_{m k+1} \cap C_{0}$ are paramterized by $\varphi\left(\frac{l}{2^{m}}\right)$ for $0 \leq l<2^{m}$. Then $V_{m k+j} \cap C_{0}=V_{m k+1} \cap C_{0}$ for $j=1, \ldots, k$. In other words, no new points in $C_{0}$ are created in the next $k-1$ subdivisions, because the intervals in the external ray parametrization between consecutive points in $C_{0}$ are the smallest intervals in $V_{m}^{\prime}$. For a fixed value of $l$, the neighbors of $x=\varphi\left(\frac{1}{2^{m}}\right)$ in $V_{m k+j} \cap C_{0}$ are exactly $\varphi\left(\frac{l-1}{2^{m}}\right)$ and $\varphi\left(\frac{l+1}{2^{m}}\right)$. Of course $x$ will have $2 k-2$ neighbors in each of the graphs 
$\Gamma_{m k+j}$ that lie on the loops that attach at $x$, and these neighbors will vary with $j$. For each loop there will be exactly four such neighbors. In $\Gamma_{m}$ we denote the neighbors $y_{1}, \ldots, y_{k-1}$ and $z_{1}, \ldots, z_{k-1}$, where $y_{j}$ and $z_{j}$ lie on the same loop and have distance $\frac{1}{\left(2^{k}-1\right) 2^{m k+j}}$ from $x$ in the external ray parametrization. These $\left(y_{j}\right.$ and $z_{j}$ ) are also neighbors in $\Gamma_{m k+2}, \Gamma_{m k+3}, \ldots, \Gamma_{m k+j}$, but in $\Gamma_{m k+j+1}$ their intervals get subdivided and we obtain new neighbors $y_{j}^{\prime}$ and $z_{j}^{\prime}$ in the same loop at distance $\frac{1}{\left(2^{k}-1\right) 2^{(m+1) k+j}}$ to $x$. These remain neighbors in $\Gamma_{m k+l}$ for $j<l \leq k$.

Recall that we are using the discrete energy $\frac{1}{k}\left(\mathcal{E}_{m k+1}(u, v)+\mathcal{E}_{m k+2}(u, v)+\ldots+\right.$ $\left.\mathcal{E}_{(m+1) k}(u, v)\right)$ to approximate $\mathcal{E}(u, v)$, and the weight assigned to each point in $V_{(m+1) k}$ to approximate $\mu$ is $2^{-(m+1) k}$. Thus the approximate Laplacian $\Delta_{(m+1) k}$ is given by

$$
-\Delta_{(m+1) k} u(x)=2^{(m+1) k} \frac{1}{k} \sum_{j=1}^{k} 2^{\left(\frac{1-k}{k}\right)(m k+j)} E_{m k+j}(u, v),
$$

where $v$ is defined on $V_{(m+1) k}$ by $v(y)=\delta_{x y}$. Now

$$
\begin{aligned}
E_{m k+1}(u, v) & =\left(2^{k}-1\right) 2^{m k+1}\left[\left(2 u(x)-u\left(\varphi\left(\frac{l+1}{2^{m}}\right)\right)-u\left(\varphi\left(\frac{l-1}{2^{m}}\right)\right)\right)\right. \\
& \left.+\sum_{p=1}^{k-1} 2^{-p}\left(2 u(x)-u\left(y_{p}\right)-u\left(z_{p}\right)\right)\right]
\end{aligned}
$$

and in general, for $1 \leq q \leq k$,

$$
\begin{aligned}
E_{m k+j}(u, v) & =\left(2^{k}-1\right) 2^{m k+1}\left[2 u(x)-u\left(\varphi\left(\frac{l+1}{2^{m}}\right)\right)-u\left(\varphi\left(\frac{l-1}{2^{m}}\right)\right)\right. \\
& +\sum_{p=1}^{k-j} 2^{-p}\left(2\left(u(x)-u\left(y_{p}\right)-u\left(z_{p}\right)\right)\right) \\
& \left.+\sum_{p=k-j+1}^{k-1} 2^{k-p}\left(2\left(u(x)-u\left(y_{p}^{\prime}\right)-u\left(z_{p}^{\prime}\right)\right)\right)\right] .
\end{aligned}
$$

Substituting (9.9) into (9.7) and collecting terms we obtain

$$
\begin{aligned}
-\Delta_{(m+1) k} u(x) & \\
= & 2^{(m+1) k+1}\left(2^{k}-1\right) \frac{1}{k}\left[\left(\sum_{j=1}^{k} 2^{j\left(\frac{1+k}{k}\right)}\right)\left(2 u(x)-u\left(\varphi\left(\frac{l+1}{2^{m}}\right)\right)-u\left(\varphi\left(\frac{l-1}{2^{m}}\right)\right)\right)\right. \\
& +\sum_{p=1}^{k-1} 2^{-p}\left(\sum_{j=1}^{k-p} 2^{j\left(\frac{1-k}{k}\right)}\right)\left(2 u(x)-u\left(y_{p}\right)-u\left(z_{p}\right)\right) \\
& \left.+\sum_{p=1}^{k-1} 2^{k-p}\left(\sum_{k-p+1}^{k} 2^{j\left(\frac{1-k}{k}\right)}\right)\left(2 u(x)-u\left(y_{p}^{\prime}\right)-u\left(z_{p}^{\prime}\right)\right)\right] .
\end{aligned}
$$

This, of course, can be simplified. But the key point is that if $u$ is a $\lambda$ eigenfunction where $\lambda$ satisfies the assumption (9.6), we can use (9.5) to express all the terms $\left.u\left(y_{p}\right), u\left(z_{p}\right), u_{(} y_{p}^{\prime}\right)$, and $u\left(z_{p}^{\prime}\right)$ as multiples of $u(x)$. Thus

$$
-\Delta_{(m+1) k} u \approx \lambda u
$$


may be written as

$$
2^{(m+1) k} A_{k}\left(2 u(x)-u\left(\varphi\left(\frac{l+1}{2^{m}}\right)\right)-u\left(\varphi\left(\frac{l-1}{2^{m}}\right)\right)\right) \approx\left(\lambda+B_{k}(x, m, \lambda)\right) u(x)
$$

for constants $A_{k}$ and $B_{k}$. Note that $B_{k}$ depends on $x$ because the lengths of the loops that attach to $x$ vary. More precisely, it depends only on the level where $x$ first appears. $B_{k}$ also depends on $\lambda$ via (9.5), and this is a nonlinear dependence. Of course (9.12) is only approximately true, and what we are interested in is the limit as $m \rightarrow \infty$. We expect that the limit will be an eigenvalue equation (the eigenvalue will depend on $\lambda$ in a nonlinear fashion) for $u \circ \varphi$ for a Laplacian built from the standard energy and some new measure that also depends on $\lambda$.

A simple special case is that of $\lambda=0$, or harmonic functions. While there are no global harmonic functions on $\mathcal{J}$, there are piecewise harmonic functions that enable us to describe the Green's function. Let us fix two points $z_{0}, z_{1}$ in $\mathcal{J}$, and to begin we will assume that both are points on the same circle $C_{n}$ of length $L_{n}$, $z_{0}=\varphi_{n}\left(t_{0}\right), z_{1}=\varphi_{n}\left(t_{1}\right)$. We construct a function $G(t)$ on the parameter circle so that $-G^{\prime \prime}=\delta_{t_{1}}-\delta_{t_{0}}$. This only determines $G$ up to an additive constant, so we adjoin the arbitrary condition $G\left(t_{0}\right)=0$. It is easy to see that

$$
G(t)=\left\{\begin{array}{ccc}
\frac{1}{L_{n}}\left(t-t_{0}\right)\left(L_{n}-t_{1}+t_{0}\right) & \text { if } & t_{0} \leq t \leq t \\
\frac{1}{L_{n}}\left(L_{n}-t+t_{0}\right)\left(t_{1}-t_{0}\right) & \text { if } & t_{1} \leq t \leq L_{n}+t_{0}
\end{array}\right.
$$

(here $G$ is extended to be periodic of period $\left.L_{n}\right)$. Note that $G\left(t_{0}\right)=G\left(L_{n}+t_{0}\right)=0$ and both expressions give $G\left(t_{1}\right)=\frac{\left(t_{1}-t_{0}\right)\left(L_{n}-t_{1}+t_{0}\right)}{L_{n}}$, so $G$ is a piecewise linear and continuous, and the jumps in $G^{\prime}$ yield $-G^{\prime \prime}=\delta_{t_{1}}-\delta_{t_{0}}$.

Next we transfer $G$ to $\mathcal{J}$ by defining

$$
\tilde{G}\left(\varphi_{n}(t)\right)=G(t)
$$

and making $\tilde{G}$ constant on all loops that attach to $C_{n}$. We may give a more explicit description of $\tilde{G}$ in terms of the external ray parametrization. The circle $C_{n}$ is parameterized by a pair of Cantor sets whose extremities are identified, together with some discrete points (when $k \geq 3$ ) identified with some points on the Cantor set. Call this set $C_{n}^{\prime}$. The complement of $C_{n}^{\prime}$ in the external ray parameter circle is a countable union of intervals $\cup I_{j}$, where the endpoint $a_{j}, b_{j}$ of each $I_{j}$ are identified. Of course the interval $I_{j}$ parametrizes the interior of a loop that attaches at the endpoints, so

$$
\tilde{G}(x)=\tilde{G}\left(a_{j}\right) \text { for } x \in I_{j}, \quad a_{j} \in C_{j} .
$$

Note that (9.14) and (9.15) together define $\tilde{G}$ on $\mathcal{J}$. To show the dependence on $z_{0}, z_{1}$ and $n$ we write $\tilde{G}_{n}\left(z_{0}, z_{1}\right)(x)$.

Because $\tilde{G}$ is constant on loops, only the first term in (9.10) is nonzero, so

$$
-\Delta_{(m+1) k} \tilde{G}_{0}\left(z_{0}, z_{1}\right)(x)=B_{k} 2^{m k}\left(2 G\left(\frac{l}{2^{m}}\right)-G\left(\frac{l+1}{2^{m}}\right)-G\left(\frac{l-1}{2^{m}}\right)\right)
$$

if $x=\varphi_{0}\left(\frac{l}{2^{m}}\right)$, and $-\Delta_{(m+1) k} \tilde{G}_{0}\left(z_{0}, z_{1}\right)$ vanishes outside $C_{0}$. Thus

$$
-\Delta_{\mu} \tilde{G}_{0}\left(z_{0}, z_{1}\right)=B_{k}\left(\delta_{z_{1}}-\delta_{z_{0}}\right) .
$$

A similar expression holds for any circle $C_{n}$. 
We may now construct a Green's function $G_{z}(x)$ for any point $z \in \mathcal{J}$ that satisfies

$$
-\Delta_{\mu} G_{z}=\delta_{z}-\delta_{z_{0}} \text { and } G_{z}\left(z_{0}\right)=0
$$

where $z_{0}=\varphi_{0}(0)$ is a fixed base point. For simplicity we first describe the case where $z$ belongs to a circle. Then there is a unique chain of circles $C_{0}, C_{n_{1}}, C_{n_{2}}, \ldots$, $C_{n_{N}}$ and points $z_{0}, z_{1}, \ldots, z_{N}=z$ such that $z_{j}$ and $z_{j+1}$ both belong to $C_{j}$ (this means $z_{j}=C_{j-1} \cap C_{j}$ for $\left.0<j<N\right)$. It is easy to see that

$$
G_{z}=\frac{1}{B_{k}}\left(\tilde{G}_{0}\left(z_{0}, z_{1}\right)+\sum_{j=1}^{N}\left(\tilde{G}_{n_{j}}\left(z_{j}, z_{j+1}\right)-\tilde{G}_{n_{j}}\left(z_{j}, z_{j+1}\right)\left(z_{0}\right)\right)\right)
$$

satisfies (9.18). If $z$ does not belong to a circle, then there is a unique infinite chain of circles and points such that $z_{n} \rightarrow z$ as $N \rightarrow \infty$, and then (9.19) holds with $N=\infty$. Note that $G_{z}$ is a piecewise linear function on circles.

We can use the Green's function to "invert" the Laplacian as follows. Since the Laplacian is neither one-to-one nor onto, we impose the condition

$$
\int_{\mathcal{J}} f(y) d \mu(y)=0
$$

on the right side of

$$
-\Delta_{\mu} u=f
$$

to assure existence, and we assume

$$
u\left(z_{0}\right)=0
$$

to determine a unique solution. The solution is then given by integration against $G_{z}$,

$$
u(x)=\int_{\mathcal{J}} G_{y}(x) f(y) d \mu(y) .
$$

Indeed,

$$
\begin{array}{r}
-\Delta_{\mu} u(x)=\int_{\mathcal{J}}\left(\delta_{y}(x)-\delta_{z_{0}}(x)\right) f(y) d \mu(y) \\
=f(x)-\left(\int_{\mathcal{J}} f(y) d \mu(y)\right) \delta_{z_{0}}(x) \\
=f(x)
\end{array}
$$

by $(9.18)$ and $(9.20)$, and $u\left(z_{0}\right)=0$ by (9.18).

We can also solve

$$
-\Delta_{\mu^{\prime}} u=f
$$

and (9.22) under the assumption

$$
\int_{\mathcal{J}} f(y) d \mu^{\prime}(y)
$$

by

$$
u(x)=\int_{\mathcal{J}} G_{y}(x) f(y) d \mu^{\prime}(y)
$$

for any finite Borel measure on $\mathcal{J}$ that assigns positive measure to open sets. 


\section{ACKNOWLEDGEMENTS}

The authors would like to thank Jun Kigami, John H. Hubbard, Luke Rogers, and John Smillie for many helpful conversations.

\section{REFERENCES}

[ADS10] Tarik Aougab, Chu Yue (Stella) Dong, and Robert S. Strichartz, Laplacians of a family of quadratic julia sets II, Comm. Pure Appl. Math., to appear.

[ASST03] Bryant Adams, S. Alex Smith, Robert S. Strichartz, and Alexander Teplyaev, The spectrum of the Laplacian on the pentagasket, Fractals in Graz 2001, Trends Math., Birkhäuser, Basel, 2003, pp. 1-24. MR2091699 (2006g:28017)

[Bar98] Martin T. Barlow, Diffusions on fractals, Lectures on probability theory and statistics (Saint-Flour, 1995), Lecture Notes in Math., vol. 1690, Springer, Berlin, 1998, pp. 1121. MR1668115 (2000a:60148)

[Bra89] Bodil Branner, The Mandelbrot set, Chaos and fractals (Providence, RI, 1988), Proc. Sympos. Appl. Math., vol. 39, Amer. Math. Soc., Providence, RI, 1989, pp. 75-105. MR1010237

[CSW09] Sarah Constantin, Robert S. Strichartz, and Miles Wheeler, Analysis of the Laplacian and spectral operators on the Vicsek set, Comm. Pure Appl. Anal. 10 (2011), no. 1, 1-44. MR 2746525

[DH84] Adrien Douady and John H. Hubbard, Etude dynamique des polynomes complexes $i$, Publ. Math. d'Orsay (1984).

[Flo08] Taryn C. Flock, Laplacians on a family of quadratic julia sets, http://www.math. cornell.edu/ taryn/data.html, September 2008.

[FS92] M. Fukushima and T. Shima, On a spectral analysis for the Sierpiński gasket, Potential Anal. 1 (1992), no. 1, 1-35. MR1245223 (95b:31009)

[Kig01] Jun Kigami, Analysis on fractals, Cambridge Tracts in Mathematics, vol. 143, Cambridge University Press, Cambridge, 2001. MR.1840042 (2002c:28015)

[KL93] Jun Kigami and Michel L. Lapidus, Weyl's problem for the spectral distribution of Laplacians on p.c.f. self-similar fractals, Comm. Math. Phys. 158 (1993), no. 1, 93125. MR1243717 (94m:58225)

[RT09] Luke G. Rogers and Alexander Teplyaev, Laplacians on the Basilica julia sets, Comm. Pure Appl. Anal. 9 (2010), no. 1, 211-231. MR2556753 (2011c:28024)

[Str06] Robert S. Strichartz, Differential equations on fractals, Princeton University Press, Princeton, NJ, 2006, A tutorial. MR2246975 (2007f:35003)

[Zho09] Denglin Zhou, Spectral analysis of laplacians on viscek sets, Pac. J. Math. 241 (2009), 369-398.

[Zho10] Criteria for spectral gaps of Laplacians on fractals, J. Fourier Anal. Appl. 16 (2010), no. 1, 76-96. MR2587582(2011b:28026)

Department of Mathematics, University of California Berkeley, Berkeley, CaliforNIA 94709

E-mail address: tflock@math.berkeley.edu

Department of Mathematics, Malott Hall, Cornell University, Ithaca, New York 14853

E-mail address: str@math.cornell.edu 